\title{
Characterization of hydrogenated silicon thin films and its alloys by the photoconductivity frequency mixing and transient thermoelectric effects methods
}

\author{
Dissertation \\ zur Erlangung des Doktorgrades \\ der Mathematisch-Naturwissenschaften Fakultäten \\ der Georg-August-Universität zu Göttingen
}

vorgelegt von

Mostafa Abd EIMoemen Hassan Boshta

aus (El Menofia) Ägypten

Göttingen, 2003 


\section{CONTENTS}

\section{Chapter 1: INTRODUCTION}

1.1. Introduction 1

1.2. Hydrogenated polycrystalline silicon thin films 2

1.3. Hydrogenated amorphous silicon germanium thin films 4

1.4. Hydrogenated microcrystalline silicon germanium thin films 5

1.5. The preparation techniques 6

\section{Chapter 2: EXPERIMENTAL WORK}

2.1. The Photoconductivity frequency mixing technique 11

2.1.1. Introduction 11

2.1.2. Theory of the photomixing 11

$\begin{array}{lll}\text { 2.1.3. } & \text { Experimental setup for photomixing } & 18\end{array}$

2.1.4. Detection of the microwave photomixing signal 19

2.1.4.1. Tuning stub 19

2.1.4.2. A typical experiment 20

2.1.4.3. Improvement of the photomixing instrumentation 20

2.2. Transient thermoelectric effects (TTE) 23

2.2.1. The theory of the TTE method 25

2.2.2. Ambipolar charge distribution 25

2.2.3. Calculation of the stage $\mathrm{II}_{1}$ relaxation time $\tau_{2} \quad 26$

2.2.4. Calculation of the relaxation amplitude $\mathrm{V}_{20}$ of stage $\mathrm{II}_{1}$

2.2.5. Transient Seebeck-effect 30

2.2.6. Calculation of the relaxation time $\tau_{2}, \quad 30$

2.2.7. Calculation of the relaxation amplitude $\mathrm{V}_{\mathrm{s}}$

2.2.8. Trap density of state as extracted for $\mathrm{V}_{20}$

2.2.9. Experimental setup for TTE-measurements 33

2.2.10. Extraction of $\mathrm{V}_{2}(\mathrm{t}) \quad 34$

2.3. Photoluminescence and small angle X-ray scattering (SAXS) 37 
2.3.1. Photoluminescence 37

2.3.2. Small angle X-ray scattering (SAXS) 38

2.4. Sample preparation 41

2.4.1. The hot wire chemical vapor deposition poly-Si:H high deposition 41 rate samples

2.4.2. The hot wire chemical vapor deposition (HWCVD) a-SiGe:H alloys 42

2.4.3. The low pressure reactive ECR plasma $\mu \mathrm{c}-\mathrm{SiGe} \mathrm{H}$ samples 43

\section{Chapter 3: RESULTS}

3.1. The photomixing results 45

3.1.1. Determination of the range and depth of long-range potential 45 fluctuations

3.1.2. Estimate of the charged defect density 46

3.1.3. Application to the samples 46

3.1.3.1 The hot wire chemical vapor deposition poly-Si:H high deposition 46 rate samples

3.1.3.2 The hot wire chemical vapor deposition a-SiGe:H samples 52

3.1.3.3 The low pressure reactive ECR plasma $\mu \mathrm{c}-\mathrm{SiGe}: \mathrm{H}$ samples $\quad 54$

3.2. The TTE-results 56

3.2.1. The hot wire chemical vapor deposition poly-Si:H high deposition 56 rate samples

3.2.2. The hot wire chemical vapor deposition a-SiGe:H samples 60

3.2.3. The low pressure reactive ECR plasma $\mu \mathrm{C}-\mathrm{SiGe}: \mathrm{H}$ samples 64

3.3. Photoluminescence and small angle X-ray scattering (SAXS) 67

\section{Chapter 4: DISCUSSION}

4.1. Transport properties of poly-Si:H thin films 72

4.1.1. Transport properties of the hot wire chemical vapor deposition poly- 72 $\mathrm{Si}: \mathrm{H}$

4.2. Transport properties of the hot wire chemical vapor deposition a- 74 SiGe:H samples 
4.3. Transport properties of the low pressure reactive ECR plasma $\mu \mathrm{c}-76$ SiGe:H samples

$\begin{array}{lll}\text { 4.4. Space charges in poly-Si:H films } & 78\end{array}$

4.4.1 The hot wire chemical vapor deposition a-SiGe:H samples 79

4.4.2 The low pressure reactive ECR plasma $\mu \mathrm{C}$-SiGe:H samples $\quad 80$

4.5. The photoluminescence and SAXS characterizations 80

4.6. The correlation between the photomixing and TTE-results 81

CONCLUSIONS 83

REFERENCES $\quad 85$

$\begin{array}{ll}\text { Acknowledgements } & 93\end{array}$ 


\subsection{Introduction}

\section{Introduction}

Hydrogenated amorphous silicon (a-Si:H) has received extensive interest since its successful fabrication by the Dundee group which first used the rf glow discharge method in 1976[1]. It is appealing not only for its low cost and low processing temperature, but also for its potential use in large area electronic devices, i.e., solar cells [2], thin film transistor [3], visible light emitting diodes [4], color detectors [5], and novel edge and orientation detectors [6]. Since its optical and electrical properties can be tailored by alloying with other materials such as carbon (a-SiC:H)[7-11], and germanium (aSiGe:H)[12-14] hydrogenated amorphous silicon based alloys can also be used for very specific applications. As an example, the hydrogenated amorphous silicon germanium alloy (a-SiGe:H) is especially suitable for the use in terrestrial solar cells due to its low optical gap. Some important questions remain about the structural and electrical properties of the a-Si:H materials and its alloys.

In this work the polycrystalline silicon and a-SiGe:H samples were prepared by the hot wire chemical vapor deposition technique with some improvement to have high quality materials. Also, $\mu \mathrm{c}-\mathrm{SiGe}: \mathrm{H}$ films were prepared by the low pressure ECR plasma technique for the first time. We investigated the charge transport properties of such materials by using the photoconductivity frequency mixing technique. The photomixing technique has been employed to measure the photoconductivity $(\sigma)$, drift mobility $(\mu)$ and lifetime $(\tau)$ of these thin film materials as a function of deposition rate and/or the Tauc gap. This method, which is different from either Hall measurements or time of flight (TOF) methods, has unique advantages to measure semiconductors materials with high resistance and low mobility in that it measures $\mu$ and $\tau$ of a given samples simultaneously. Also, the transient thermoelectric effects technique was employed for such materials. The transient thermoelectric effects technique, in which a pulsed laser light is irradiated upon one end of a sample, one measures an induced voltage across both ends of the sample and analyzes its decay profile. From the analysis we can obtain valuable information about electron-hole generation/ recombination processes, space charges and thermal diffusion of photo-generated carriers (and phonons) drifting along 
the concentration and temperature gradient in the sample. This technique is particularly useful for multi-carrier systems, i.e. if we have both conduction electrons and holes.

\subsection{Hydrogenated polycrystalline silicon thin films}

Polycrystalline silicon is used today for many thin film applications and has been the subject of intense research for almost three decades.

In particular, thin film polycrystalline silicon is used for applications such as silicon gate transistors, nonvolatile memories, passive resistors, local interconnects, bipolar transistor diodes, dynamic access memories, solar cells and micromechanical systems. The inherently varying structural and electrical properties of polycrystalline silicon at the length scale of individual grains affect these applications to different degrees. Microelectronics applications are particularly susceptible to problems which result from the small-scale variability of polycrystalline silicon because the dimensions of many microelectronic devices are near the average grain size of thin film polycrystalline silicon, typically a few tenths of a micron. These problems are expected to worsen in the future as devices are scaled down further in an attempt to improve speed and performance.

In order to control the variability of electrical properties of polycrystalline silicon it is necessary first to understand how the grain structure fundamentally determines the electrical properties. Once the relationship between grain structure and electrical properties has been elucidated, it may be possible to use process variables such as deposition pressure or annealing time to directly control the distribution of electrical properties. Even applications which use hundreds or thousands of grains (such as solar cells or micromechanical systems) would benefit from such an understanding since it may be possible to more effectively alter the average properties of polycrystalline silicon as well as the variance of those properties.

In studies of polycrystalline silicon thin films, it has been established that the properties of the boundaries between adjacent grains (as opposed to the properties of the single grains themselves) are most important for understanding the electrical properties of the film [22-26]. Atomic level disorders at the boundary between grains introduce defect states, which may be filled with trapped charge. The trapped charge then causes energy band bending to occur and introduces energy barriers to current transport, shown in 
Fig.(1-1). In real blanket films, however, all barriers are not identical as shown. The distribution of grain boundary structures fundamentally determines the distribution of barrier heights in polycrystalline silicon thin films. However, there have been no quantitative surveys of either the distribution of the grain boundaries or the convolution of grain boundary resistances. Further, a one-to-one correspondence between the grain boundary structure and the electrical properties has not been demonstrated for deposited submicron grains in thin films, which makes it difficult to find theoretical equations which master the description of the experiments.

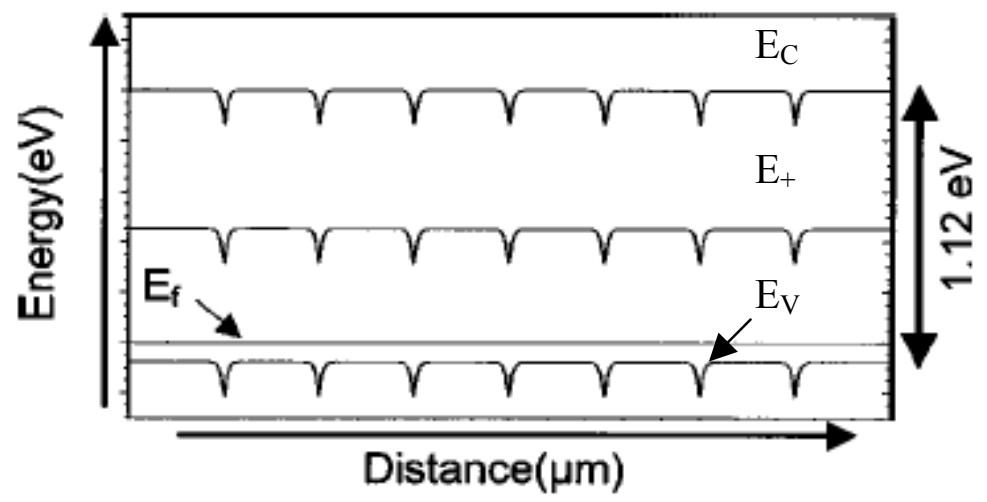

Fig.(1-1) Schematic band bending with trapped charges at the grain boundary for a boron-doped film; in a real film not all barriers are identical as shown. According to [27]

Limitations such as photo degradation, limited long wavelength photosensitivity and the low carrier mobility of hydrogenated amorphous silicon (a-Si:H) films prevent the use of this material in a large number of applications [20]. A possible solution that is receiving considerable attention is the development of polycrystalline silicon (poly-Si) films with suitable properties. Because of its high photosensitivity in the long-wavelength region and the possibility of a high carrier mobility, poly-Si can be used for solar cells and other optoelectronic devices [21]. Usually a high processing temperature $\left(>600{ }^{\circ} \mathrm{C}\right)$ is used in order to get polycrystalline films with a large grain size. But the high temperature constrains the use of low-cost substrates. So the challenge was to prepare polycrystalline silicon at low temperatures. To grow high-quality poly-Si films, different chemical vapor deposition (CVD) processes like catalytic-CVD, low-pressure CVD, 
electron cyclotron resonance CVD, plasma enhanced chemical vapor deposition (PECVD) etc [22-25] and non-CVD processes like radio frequency (rf) magnetron sputtering [26] are being tried to grow device quality poly-Si films.

\subsection{Hydrogenated amorphous silicon germanium thin films}

Hydrogenated amorphous, germanium alloys (a-SiGe:H) have attracted considerable attention as promising new materials for photovoltic and opto-electronic devices[28,29]. Alloys with $\approx 40$ at. $\%$ Ge have a band gap of $\approx 1.4 \mathrm{eV}$ which has made them useful as an active layer material in photovoltic (PV) tandem cells. Alloys with higher Ge content and smaller band gap to $\approx 1.2 \mathrm{eV}$ are also of interest for PV applications, however, the transport properties of $\mathrm{a}-\mathrm{Si}_{\mathrm{x}} \mathrm{Ge}_{1-\mathrm{x}}: \mathrm{H}$ films degrade with respect to those of a-Si:H, with the degree of degradation increasing as (i) the band gap decreases and (ii) the Ge content increases. This has prompted research on hydrogenated microcrystalline silicon, germanium alloys $\left(\mu \mathrm{c}-\mathrm{Si}_{\mathrm{x}} \mathrm{Ge}_{1-\mathrm{x}}: \mathrm{H}\right)$ as possible alternative material for PV devices.

a-SiGe:H is a tetrahedrally bonding semiconductor just like a-Si:H. It has also been suggested that $\mathrm{Si}-\mathrm{H}_{2}$ configurations in a SiGe:H films cause the deterioration of the photoelectronic properties of the materials and solar cell performances, including a limited stability against light exposure[30,31]. However, the $\mathrm{H}$ bonding structure in aSiGe:H is much less understood than that of a-Si:H due the tendency of $\mathrm{H}$ atoms to selectively bond to Si rather than to Ge, so-called "preferential attachment"[32-34]. This selective $\mathrm{H}-\mathrm{Si}$ bonding is believed to cause a microscopic inhomogeneity in the network and to deteriorate the electronic properties of the alloys. The preferential attachment is probably due to the smaller binding energy of $\mathrm{H}-\mathrm{Ge}$ than that of $\mathrm{H}-\mathrm{Si}$. However, the detailed mechanisms governing the preference of $\mathrm{H}$ bonding and deterioration of material properties are not yet completely clear.

After the first development of the a-SiGe:H alloy by decomposing a silane and germaine mixture in glow discharge[35], even now different deposition techniques like radio frequency (rf) or direct current (dc) glow discharge, rf sputtering, photochemical vapor deposition (CVD), microwave-CVD[36-39], as well as different source and diluent gases[40,41] are being used to improve the opto-electronic properties of a-SiGe:H alloys. 
Hot wire chemical vapor deposition HW-CVD techniques have received considerable attention in recent years as an alternative deposition method for a-Si:H because it is capable of improving film stability[42] and of achieving higher deposition rates[43]. In HW-CVD, no discharge has to be sustained and only particles with thermal energies are involved. In contrast to the P-CVD process, the advantage of this technique is its extreme simplicity. So, the capital cost and production cost of thin film production expected to be the lowest among all the techniques. To our knowledge, this technique has not been studied extensively for the synthesis of a-SiGe:H alloy films and there are only few reports existing in the literature $[44,45]$. Hence, immediate action to investigate the synthesis and characterization of a-SiGe:H films is suggested.

\subsection{Hydrogenated microcrystalline silicon germanium thin films}

Crystalline silicon (c-Si) solar cells suffer from an intrinsically low optical absorption coefficient due to the indirect band structure of the semiconductor. A total thickness of approximately $50 \mu \mathrm{m}$ is necessary to effectively absorb the sunlight in a c-Si solar cell. The use of optimized light trapping structures can reduce the required absorber thickness to approximately $20 \mu \mathrm{m}$ [46]. However, the preparation of a wafer with only 20 $\mu \mathrm{m}$ thickness remains a technological challenge. Up to now, it has been impossible to achieve such small thicknesses with a satisfactory yield by conventional wafer sawing techniques. On the other hand, the epitaxial growth of thin c-Si films has been mainly limited by low growth rates and the lack of suitable low temperature substrates.

An alternative route to efficient c-Si based solar cells has been the use of $\mu \mathrm{c}-\mathrm{Si}: \mathrm{H}$ (microcrystalline-Si), which can be directly grown on glass covered with a transparent conducting oxide. Because the absorption of $\mu \mathrm{c}-\mathrm{Si}: \mathrm{H}$ is slightly higher than that of c-Si, a thickness of 1-3 $\mu \mathrm{m}$ in conjunction with efficient light trapping is usually enough to absorb most of the sunlight. So far, such thin film solar cells based on $\mu \mathrm{c}-\mathrm{Si} / \mathrm{a}-\mathrm{Si}$ tandem structures have achieved efficiencies up to $11 \%$ [47]. Major problems remaining for the use of $\mu \mathrm{c}-\mathrm{Si}$ as the absorber material in thin-film Si-based solar cells are the low absorption coefficient as well as the low growth rates of high-quality $\mu \mathrm{c}-\mathrm{Si}$ layers in PECVD. Here, the use of strongly absorbing crystalline SiGe-thin films with a suitably adjusted Ge content may provide alternative solutions for the near future. 
Recently, the growth processes and microstructures of hydrogenated microcrystalline silicon-germanium alloys ( $\mu \mathrm{c}-\mathrm{SiGe}: \mathrm{H})$ have been widely investigated since these materials can be utilized in thin film transistors (TFTs), thin film solar cells as bottom cell active layer materials and other thin film opto-electronic devices. Compared with their amorphous counterparts, $\mu \mathrm{c}-\mathrm{SiGe}: \mathrm{H}$ have several advantages such as higher conductivity $(\sigma)$, mobility $(\mu)$, stronger absorption in the near-infrared region and longer stability against light induced degradation[48,49]. A lot of work has been done on how to fabricate high-quality $\mu \mathrm{c}-\mathrm{SiGe}: \mathrm{H}$ films and how to improve their electronic transport properties through controlling deposition parameters such as hydrogen dilution, substrate temperature and so on[50-52]. In 1996, Ganguly successfully fabricate high-quality $\mu \mathrm{c}-$ SiGe:H alloy and the growth process, microstructures, optical properties were systematically studied[53,54]. However, the electronic transport properties of $\mu \mathrm{c}-\mathrm{SiGe}: \mathrm{H}$ films still remains unclear especially in the samples deposited under very high hydrogen dilution and higher substrate temperature conditions, though it is very important for the performance of actual devices.

\subsection{The preparation techniques:}

Because progress in the tailoring can be made by improving the preparation techniques, in this chapter, we go into the different preparation techniques. Different process have been used: (1) plasma enhanced chemical vapor deposition (PECVD), (2) chemical vapor deposition (CVD) processes like catalytic-CVD, (3) electron cyclotron resonance (ECR)- CVD, (4) Microwave CVD, and (5) plasma beam deposition.

\section{Plasma enhanced chemical vapor deposition: (1)}

Amorphous and polycrystalline silicon thin films are still commonly produced using the method that first resulted in hydrogen incorporation in the material [55]. The deposition method is a glow discharge technique, also known as Plasma Enhanced Chemical Vapor Deposition (PECVD). A silicon containing gas, usually silane $\left(\mathrm{SiH}_{4}\right)$, is admitted to a vacuum reactor chamber. A gas discharge is then initiated and maintained by an electric field between two parallel plates, using either a dc voltage or ac voltage in the radio frequency domain $(13.65-200 \mathrm{MHz})$. The pressure is typically $0.1-1 \mathrm{mbar}$, 
depending on the geometry of the vacuum chamber. The hydrogen bonded to silicon was later recognized to being essential for bonding the unpaired valence electrons that would otherwise lead to electronic defect states.

The deposition process by an rf discharge can be described as a four step process.

1- The primary reaction in the gas phase is electron-impact excitation, dissociation, and ionization of the $\mathrm{SiH}_{4}$ molecules. The plasma thus consists of neutral radicals and molecules, positive as well as negatives ions, and electrons.

2- Secondary reactions, between molecules and ions or radicals, are very important as they predominantly control the electronic and structural film properties. Reactive neutral species move to the substrate by diffusion, positives ions bombard the growing film, and negative ions are trapped within the sheaths [56] and may eventually form small particles, or dust.

3- The third step consists of surface reactions, such as hydrogen abstraction, radical diffusion, and chemical bonding.

4- The fourth step is the subsurface release of hydrogen molecules and relaxation of the silicon matrix.

The deposition process is very complicated as the physical and chemical interactions in the plasma and at the growing film surface are dependent on the rf power and frequency, the substrate temperature, the gas pressure and composition, the magnitude and pattern of the gas flow, the electrode geometry, etc. More detail is found in the large reviews $[57,58]$.

\section{Hot- Wire-Chemical Vapor Deposition (2)}

The method of catalytic decomposition of silane or silane/hydrogen mixtures at a resistively heated filament (nowadays generally called "Hot wire chemical vapor deposition"; HWCVD) has been utilized to produce thin films of polycrystalline and amorphous silicon at relatively low temperatures on a variety of substrates.

A schematic cross section of an HWCVD set up is shown in Fig (1-2). The substrate is placed a few centimeters from the filament. The wire used as the hot filament is made of tungsten or tantalum and has a thickness between 0.25 and $0.5 \mathrm{~mm}$. The wire temperature is preferably kept between $1750^{\circ} \mathrm{C}$ and $1950^{\circ} \mathrm{C}$. Lower temperatures lead to 
tungsten silicide formation on the wires, thus reducing the catalytic action of the hot filament. Higher temperatures lead to evaporation of the tungsten wire itself and subsequent detectable tungsten incorporation in the film. By employing a proper multiwire arrangement it is possible to obtain good thickness uniformity over a large area [59].

The gas flow is chosen as to prevent depletion of silane. The gas pressure and gas source composition (e.g., silane diluted in hydrogen) have a large influence on the nature of the precursors and the structure of the deposition film.

Good quality a-Si:H films are generally obtained from pure silane, although a source gas of silane diluted to $1 \%$ in He also lead to good material properties [60]. Good quality poly-Si:H films are obtained when the silane is diluted in hydrogen at higher pressure. The hot filament decomposes all hydrogen molecules into reactive atoms that effectively etch silicon from disordered or strained bonding sites, leading to a transition from an amorphous network to a crystalline network. The reason why HWCVD is so much more successful in producing polycrystalline films without any amorphous tissue than PECVD which uses highly diluted silane is that the filament is a much more effective source for atomic hydrogen than a glow discharge [61].

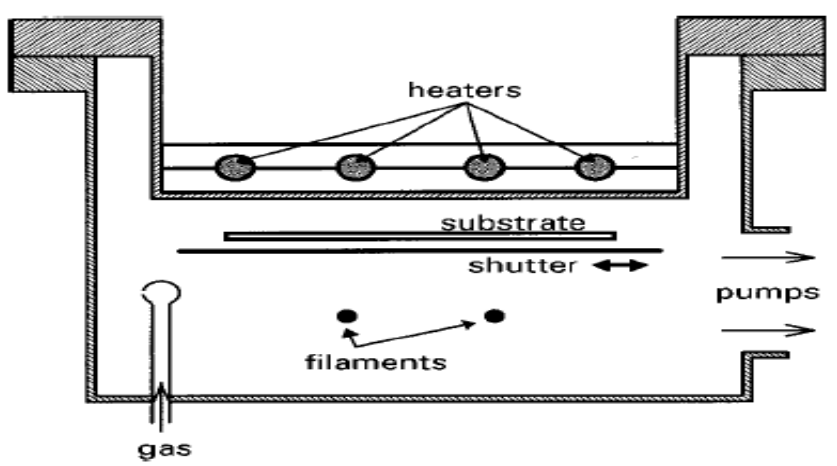

Fig.(1-2) Cross-sectional view of the hot-wire chemical vapor deposition chamber.

\section{Electron Cyclotron Resonance (3)}

The "Electron Cyclotron Resonance" CVD (ECR-CVD) method [62] is in fact a remote-plasma CVD method. Plasma is generated in a carrier gas such as $\mathrm{Ar}, \mathrm{He}$ of $\mathrm{H}_{2}$ by microwave excitation (usually $2.45 \mathrm{GHz}$ ) in a remote chamber. Due to an external applied magnetic field the electrons achieve cyclotron motion and by tuning the field 
strength $B$ the frequency of the electrons $\omega=\mathrm{eB} / \mathrm{m}$ is brought at resonance with the microwave frequency. Thus the remote plasma chamber acts as a resonance cavity and hence the method is called Electron Cyclotron Resonance CVD. The microwave power is rather efficiently transferred to the plasma. The plasma is characterized by a density of high-energy electrons, which leads to a high dissociation rate, while the gas temperature is low. The plasma can be sustained at pressures as low as $1 \mathrm{mTorr}$. The feedstock silane is introduced at a location near the substrate. As the magnetic field diverges into the deposition chamber, an electric field is generated in the plasma column, and ionized radicals $\left(\mathrm{SiH}_{\mathrm{n}}{ }^{+}\right)$from the plasma are driven to the substrate where they bombard the growing surface. The large ion flux allows the use of low substrate temperatures. The ion energy can be controlled by the gas pressure. Due to the significant ion energy transfer to the substrate, ECR-CVD is particularly suitable for a-SiGe:H deposition [63] and for highly conductive microcrystalline doped layered deposition [64]. Dalal et.al.,[65] proposed that more stable amorphous silicon materials could be produced using $\mathrm{H}_{2}$ dilution ECR-CVD. Recently, this technique which was introduced for intrinsic a-Si:H has been applied to $n-i-p$ solar cells [66].

\section{Microwave Chemical Vapor deposition (4)}

The method referred to as microwave CVD method can be either an afterglow CVD technique or a technique where the substrate is in direct contract with the plasma. The remote microwave CVD method is much like ECR-CVD, but a higher magnetic field is used so that the electrons are not at resonance. This method is more suitable for large area deposition as high ion densities can be achieved in a diverging plasma. Again, a low process pressure is used and high deposition rates can be achieved. An inherent drawback with respect to ECR-CVD is the decreased density of the plasma at the growing surface and the reduced gas utilization efficiency.

In contrast, the MPCVD (Microwave Plasma CVD) technique, in which the substrate is in contact with the microwave plasma, has in addition to large area capability the advantage of high deposition rates $(>100 \AA / s)$. The method is specifically interesting for large area a-SiGe:H deposition at a high deposition rate and has been developed for roll-to roll coating of conductive web substrates. 


\section{Plasma Beam Deposition (5)}

The plasma beam deposition (PBD) method can also be considered as a remote CVD technique. The method was originally in use for the high rate synthesis of diamond from methane [67] and has now been adapted for the deposition of amorphous silicon at a high deposition rate [68]. A thermal plasma is produced in an argon or argon/hydrogen mixture by a dc arc or a cascaded arc discharge. The plasma beam expands into a reactor chamber and is directed at the substrate. The feedstock silane is injected just after the troch nozzle and dissociated by a charge-exchange reaction with the ionized argon atoms,

$$
\mathrm{SiH}_{4}+\mathrm{Ar}^{+} \longrightarrow \mathrm{SiH}_{3}^{+}+\mathrm{Ar}+\mathrm{H}
$$

Subsequently, the main dissociation mechanism after expansion is the hydrogen abstraction reaction. The method is interesting because of the following features: (i) the directed plasma beam results in a high radical flux at the substrate which leads to high deposition rate, (ii) the substrate is not part of a capacitive configuration as in PECVD and therefore ions are not accelerated towards the growing surface, (iii) the flux of atomic hydrogen is high, which aids hydrogen abstraction reactions at the growing surface. Thus in many respects the PBD method resembles the HWCVD method, as the gas dissociation is more complete, which generates a high density of radicals along with an abundance of hydrogen atoms. 


\section{Experimental Work}

\subsection{The Photoconductivity Frequency Mixing Technique:}

\subsubsection{Introduction}

Braunstein`s group has developed a photomixing set-up that allows to determine both drift mobility and lifetime of amorphous and crystalline semiconductors [69-73]. In the present section we present the experimental details and the theory.

The photomixing technique is based on the idea of heterodyne detection in connection with photoconductors. When two polarized monochromatic optical beams of slightly different frequencies are incident upon a photoconductor, the generation rate of electron-hole pairs and therefore the photocurrent produced under a dc bias, will contain components resulting from the square of the sum of the incident electrical fields. Consequently, due to the beat frequency of the incident fields a photocurrent composed of a dc and a microwave current will be produced; the measurement of these two photocurrents allow a determination of the mobility and lifetime of the photogenerated carriers in the same sample simultaneously.

In the present work, instead of using two lasers, the multiple longitudinal modes of a single laser were used. In this case several microwave signals or photomixing signals with different frequencies can be generated due to the beating of the various laser longitudinal modes. Fig. (2-1) shows the photomixing spectrum obtained with a SpectraPhysics $125 \mathrm{~A}$ HeNe laser $(20 \mathrm{~mW})$ on a commercial EG\&G FND100 diode. The main frequencies of the photomixing signals are: $84 \mathrm{MHz}, 168 \mathrm{MHz}, 252 \mathrm{MHz}, \ldots 1.092 \mathrm{GHz}$. This is consistent with the result that the frequencies $\left(\mathrm{f}_{\mathrm{m}}\right)$ of the longitudinal modes of the laser are given by $f_{m}=m c / 2 L$, where $\mathrm{m}$ is an integer, $\mathrm{L}$ is the length of the laser cavity and c is the speed of light in the cavity. The variation of the intensity of each frequency component is due to the intensity distribution of the laser modes and the frequency rolloff of the diode. The signal at $252 \mathrm{MHz}$ was the one mostly used, since it has the highest intensity.

\subsubsection{Theory of the photomixing}

The phenomenon of photoconductivity originates from the generation of electronhole pairs to delocalized states by optical excitations. The electron-hole pair generation 
rate $\mathrm{G}$ is proportional to the square of the total electrical field $(\mathrm{E})$ of the incident light, which is the superposition of the electrical fields $\left(E_{m}\right)$ of all the incident beams, i.e.,

$$
\mathrm{E}=\sum_{\mathrm{m}=1}^{\ell} \mathrm{E}_{\mathrm{m}} \exp \left(\mathrm{i} \omega_{\mathrm{m}} \mathrm{t}+\mathrm{i} \Phi_{\mathrm{m}}\right)
$$

where $\omega_{\mathrm{m}}$ and $\Phi_{\mathrm{m}}$ are the angular frequency and the initial phase constant, respectively, of the $\mathrm{m}^{\text {th }}$ optical beam (or mode), and $\ell$ is the total number of the individual optical beams (or modes).

If longitudinal modes of a laser are used, then

$$
\omega_{\mathrm{m}}=\omega_{1}+2 \pi(\mathrm{m}-1) \frac{\mathrm{c}}{2 \mathrm{~L}}=\omega_{1}^{\prime}+(\mathrm{m}-1) \omega_{0}
$$

where $\omega_{1}$ is the lowest frequency of the laser modes, $m \geq 1$ is an integer, $L$ is the length of the laser cavity, and $\mathrm{c}$ is the speed of light in the cavity. For the Spectra- Physics 125A laser used, $\omega_{0}=84 \mathrm{MHz}$.

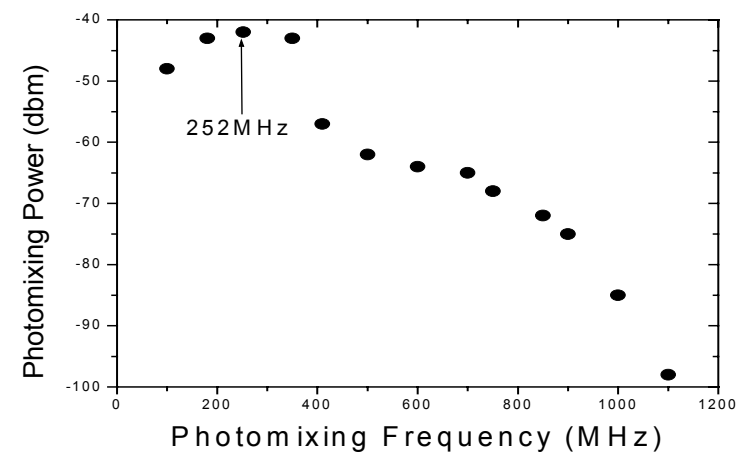

Fig.(2-1) Photomixing signal obtained with a commercial EG\&FND100 diode. The incident laser power was about $20 \mathrm{~mW}$ and the dc bias was $90 \mathrm{~V}$ (back biased). The main frequencies of the photomixing signals are $84 \mathrm{MHz}, 168 \mathrm{MHz}, 252 \mathrm{MHz}, \ldots \ldots .1 .092 \mathrm{GHz}$. The $252 \mathrm{MHz}$ was the one mostly used, since it has the highest intensity. $1 \mathrm{dbm}=1 \mathrm{~mW}$.

The total generation rate $(G)$ and the generation rate of the $\mathrm{m}^{\text {th }}$ optical beam (or mode) $\left(\mathrm{G}_{\mathrm{m}}\right)$ are proportional to the square of the corresponding electrical field, i.e. $\mathrm{G}=\mathrm{C}|\mathrm{E}|^{2}$ and $\mathrm{G}_{\mathrm{m}}=\mathrm{C}\left|\mathrm{E}_{\mathrm{m}}\right|^{2}$, where $\mathrm{C}$ is a proportionality constant. The total generation rate $\mathrm{G}$ can be further expressed as: 


$$
\begin{aligned}
& \mathrm{G}=\mathrm{C}\left|\sum_{\mathrm{m}=1}^{\ell} \mathrm{E}_{\mathrm{m}} \exp \left(\mathrm{i} \omega_{\mathrm{m}} \mathrm{t}+\mathrm{i} \Phi_{\mathrm{m}}\right)\right|^{2} \\
& =\mathrm{C} \sum_{\mathrm{m}=1}^{\ell}\left|\mathrm{E}_{\mathrm{m}}\right|^{2}+2 \mathrm{CRe}\left\{\sum_{\mathrm{m}=1 \mathrm{j}=1}^{\ell} \sum_{\mathrm{j}+\mathrm{m}}^{\ell} \mathrm{E}_{\mathrm{j}} \exp \left[\left(i \omega_{\mathrm{j}+\mathrm{m}}^{\prime}-\omega_{\mathrm{j}}\right) \mathrm{t}+\mathrm{i}\left(\Phi_{\mathrm{j}+\mathrm{m}}-\Phi_{\mathrm{j}}\right)\right],\right. \\
& =\mathrm{C} \sum_{\mathrm{m}=1}^{\ell}\left|\mathrm{E}_{\mathrm{m}}\right|^{2}+2 \mathrm{CRe}\left\{\sum_{\mathrm{m}=1}^{1-\ell} \sum_{\mathrm{j}=1}^{\ell-\mathrm{m}} \mathrm{E}_{\mathrm{j}+\mathrm{m}} \cdot \mathrm{E}_{\mathrm{j}} \exp \left[\left(\mathrm{i} \omega_{\mathrm{j}+\mathrm{m}}^{\prime}-\omega_{\mathrm{j}}\right) \mathrm{t}+\mathrm{i}\left(\Phi_{\mathrm{j}+\mathrm{m}}-\Phi_{\mathrm{j}}\right)\right]\right\}
\end{aligned}
$$

With $E_{j+m} \cdot E_{j}=\left|E_{j+m}\right|\left|E_{j}\right| \cos \left(\alpha_{j+m, j}\right)$, where $\alpha_{j+m, j}$ is the angle between the two electric fields, which can also take into account the dynamic correlation between the two laser modes. If longitudinal modes of a laser are used, then from Eq.(2-2);

$\omega_{\mathrm{j}+\mathrm{m}}^{\prime}-\omega_{\mathrm{j}}^{\prime}=\left|\mathrm{m} \omega_{0}\right|=\omega_{\mathrm{m}}$.

$$
\mathrm{G}=\mathrm{C} \sum_{\mathrm{m}=1}^{\ell} \mathrm{G}_{\mathrm{m}}+2 \mathrm{CRe}\left(\sum_{\mathrm{m}=1}^{1-\ell}\left(\sum_{\mathrm{j}=1}^{\ell-\mathrm{m}} \sqrt{\mathrm{G}_{\mathrm{j}+\mathrm{m}} \mathrm{G}_{\mathrm{m}}} \cos \left(\alpha_{\mathrm{j}+\mathrm{m}, \mathrm{j}}\right) \exp \left(\mathrm{i} \Phi_{\mathrm{j}+\mathrm{m}}-\mathrm{i} \Phi_{\mathrm{j}}\right)\right) \exp \left(i \omega_{\mathrm{m}} \mathrm{t}\right)\right)
$$

The second term on the right hand side of the above equation has to be further evaluated, since the phase constants are unknown. First, we evaluate the following quantity:

$$
\begin{aligned}
& \left|\sum_{j=1}^{l-m} \sqrt{G_{j+m} G_{j}} \cos \left(\alpha_{j+m, j}\right) \exp \left(i \Phi_{j+m}-i \Phi_{j}\right)\right|^{2}=\sum_{j=1}^{\ell-m} G_{j+m} G_{j} \cos ^{2}\left(\alpha_{j+m, j}\right) \\
& +2 \sum_{j=1}^{\ell-m} \sum_{j=1}^{\ell-m} \sqrt{G_{j+m} G_{j} G_{j+m} G_{j}} \cos ^{2}\left(\alpha_{j+m, j}\right) \cos \left(\Phi_{j^{\prime}+m}-\Phi_{j}+\Phi_{j+m}-\Phi_{j}\right) ; \quad j^{\prime}>j
\end{aligned}
$$

For laser without mode locking, the phase differences are random. Therefore, on the average, the second term on the right hand side of the above equation is much smaller than the first term and thus can be neglected to the first order. Consequently,

$$
\begin{aligned}
& \sum_{j=1}^{\ell-m} G_{j+m} G_{j} \cos \left(\alpha_{j+m, j}\right) \exp \left(i \Phi_{j+m}-i \Phi_{j}\right) \exp \left(i \omega_{m} t\right)= \\
& \sqrt{\sum_{j=1}^{\ell-m} G_{j+m} G_{j} \cos ^{2}\left(\alpha_{j+m, j}\right) \exp \left(i \delta_{m}\right)}
\end{aligned}
$$

where $\delta_{\mathrm{m}}$ is a certain phase constant. 
By defining $\quad \mathrm{G}_{0}=\sum_{\mathrm{j}=1}^{\ell-\mathrm{m}} \mathrm{G}_{\mathrm{m}} \quad$ and $\quad \lambda_{\mathrm{m}}=\sqrt{\sum_{\mathrm{j}=1}^{\ell-\mathrm{m}} \mathrm{G}_{\mathrm{j}+\mathrm{m}} \mathrm{G}_{\mathrm{j}} \cos ^{2}\left(\alpha_{\mathrm{j}+\mathrm{m}, \mathrm{j}}\right) / \mathrm{G}_{0}}$

and with the understanding that only the real part of the generation rate $G$ should be considered, $\mathrm{G}$ can be written as

$$
\mathrm{G}=\mathrm{G}_{0}+2 \mathrm{G}_{0} \sum_{\mathrm{m}=1}^{\ell-1} \lambda_{\mathrm{m}} \exp \left(\mathrm{i} \omega_{\mathrm{m}} \mathrm{t}+\mathrm{i} \delta_{\mathrm{m}}\right)
$$

$\mathrm{G}_{0}$, the total de generation rate, can be determined by the total power of the incident optical beams and can be measured by a conventional photodetector. $\lambda_{\mathrm{m}}$, the effective modulation indices, can in principle be determined by a Fabry-Perot interferometer. For a nearly linearly polarized laser beam, $\cos \left(\alpha_{j+m, j}\right) \cong 1$.

In general, especially for amorphous semiconductors and insulators, there are four processes involved in the phenomenon of photoconductivity: (1) photogeneration, (2) recombination, (3) trapping - charge carriers are trapped to the localized states inside the band gap, (4) thermal emission - trapped charge carriers are thermally emitted back to the extended states. Thus, rate equation for photo-generated electrons, which are often the dominant carriers, in the conduction band is given by $[70,71,74,75]$.

$$
\frac{\mathrm{dn}}{\mathrm{dt}}=\mathrm{G}_{\mathrm{o}}+2 \mathrm{G}_{\mathrm{o}} \sum_{\mathrm{m}=1}^{\ell-1} \lambda_{\mathrm{m}} \exp \left(\mathrm{i} \omega_{\mathrm{m}} \mathrm{t}+\mathrm{i} \delta_{\mathrm{m}}\right)-\int_{0}^{\mathrm{E}_{\mathrm{d}}} \frac{\mathrm{dn}_{\mathrm{T}}(\mathrm{E})}{\mathrm{dt}} \mathrm{dE}-\frac{\mathrm{n}}{\tau_{\mathrm{R}}}
$$

The first term on the right is the dc generation term, the second term is the ac generation from the beating of the various laser longitudinal modes with the difference frequencies of $\omega_{\mathrm{m}}$, the integral describes the number of trapped electrons in localized states below the conduction band and above the electron demarcation level $E_{d}$ due to the trapping and thermal emission processes, and the last term represents the recombination of mobile electrons with trapped holes at a constant recombination rate $1 / \tau_{R}$.

The trapped electron rate equation is given by [75]

$$
\frac{d n_{T}(E)}{d t}=n K\left[N_{T}(E)-n_{T}(E)\right]-N_{c} K n_{T} \exp \left(-\frac{E}{K T}\right)
$$

where $n_{T}(E)$ is the density of electrons in the localized states, $N_{c}$ is the effective number of states in the extended state transport band, $\mathrm{N}_{T}$ is the density of states for the conduction band tail, $K=v_{\mathrm{th}} \sigma$ ( $v_{\mathrm{th}}$ is the thermal velocity and $\sigma$ is the capture cross 
section) is the capture rate; the first term on the right represents the trapping and the second represents the thermal emission of electrons from localized gap states.

Exact solutions for Eqs.(2-8) and (2-9) can be obtained[74,75] in the form of

$$
\mathrm{n}=\mathrm{n}_{\mathrm{o}} \sum_{\mathrm{m}=1}^{\ell-1} \mathrm{n}_{\mathrm{m}} \exp \left(\mathrm{i} \omega_{\mathrm{m}} \mathrm{t}\right) \quad \text { with } \mathrm{n}_{\mathrm{o}}=\tau_{\mathrm{R}} \mathrm{G}_{\mathrm{o}} \text { and } \mathrm{n}_{\mathrm{m}}=\frac{2 \lambda_{\mathrm{m}} \mathrm{G}_{\mathrm{o}}}{\left(\mathrm{A}^{2}+\mathrm{B}^{2}\right)^{1 / 2}} \exp \left(\mathrm{i} \Phi_{\mathrm{m}}\right)
$$

where, for simplicity the mixing frequency $\omega_{\mathrm{m}}$ has been replaced by $\omega$,

$$
\begin{aligned}
& A=\frac{1}{\tau_{R}}+\int_{0}^{E_{d}} \frac{D^{2} K}{1+\exp \left[\left(E_{f n}-E\right) / k T\right]} \times \frac{\exp \left[\left(E_{f n}-E\right) / k T\right]}{D^{2}+\left\{1+\exp \left[\left(E_{f n}-E\right) / k T\right]\right\}^{2}} N_{T}(E) d E, \\
& B=\omega\left(1+\frac{1}{n_{0}} \int_{0}^{E_{d}} \frac{\exp \left[\left(E_{f n}-E\right) / k T\right]}{D^{2}+\left\{1+\exp \left[\left(E_{f n}-E\right) / k T\right]\right\}^{2}} N_{T}(E) d E\right),
\end{aligned}
$$

and $D=\frac{\omega}{N_{c} K \exp \left(-E_{f n} / k T\right)}, \quad E_{f n}=k T \ln \left(N_{c} / n_{o}\right), \quad$ and $\quad E_{\omega}=k T \ln \left(N_{c} K / \omega\right)$.

In the above equations $E_{\mathrm{fn}}$ is the quasi-Fermi level for electrons. Instead of $\omega$ and $\mathrm{n}_{0}$ one can also use $\mathrm{E}_{\mathrm{fn}}$ and the frequency-dependent demarcation energy $\mathrm{E}_{\omega}$. Their relation and physical meanings become clear if we rewrite the last two definitions as:

$$
\mathrm{n}_{\mathrm{o}}=\mathrm{N}_{\mathrm{c}} \exp \left(-\mathrm{E}_{\mathrm{fn}} / \mathrm{kT}\right) \text { and } \omega=\mathrm{N}_{\mathrm{c}} \operatorname{Kexp}\left(-\mathrm{E}_{\omega} / \mathrm{kT}\right) \text {. }
$$

Thus, roughly speaking, above $\mathrm{E}_{\omega}$ the carriers are in quasi-thermal equilibrium since their thermal emission frequencies are higher than the mixing frequency $\omega$, while those below $\mathrm{E}_{\omega}$ are in deep traps with concomitant emission frequencies less than the mixing frequency $\omega$.

When a dc bias is applied, the resulting dc and root-square ac conductivities are given by

$$
\begin{gathered}
\sigma_{\mathrm{dc}}=\mathrm{eG}_{\mathrm{o}} \mu_{\mathrm{o}} \tau_{\mathrm{R}} \\
\sqrt{\left\langle\sigma_{a c}^{2}\right\rangle}=\sqrt{2} e G_{o} \mu_{o} \frac{\lambda_{m}}{\sqrt{A^{2}+B^{2}}}
\end{gathered}
$$

where $\mu_{o}$ is the extended state mobility.

In the case of no trapping, the above equation becomes 


$$
\sqrt{\left\langle\sigma_{\mathrm{ac}}^{2}\right\rangle}=\sqrt{2} \mathrm{eG}_{\mathrm{o}} \mu_{\mathrm{o}} \frac{\lambda_{\mathrm{m}}}{\sqrt{\omega^{2}+1 / \tau_{\mathrm{R}}^{2}}}
$$

Comparing the last two equations, one can see that in general the quantity

$$
\mu_{o} \sqrt{\omega^{2}+1 / \tau_{R}^{2}} / \sqrt{A^{2}+B^{2}}
$$

plays the same role in the case of trapping as the extended state mobility $\mu_{\mathrm{o}}$ in the case of no trapping, thus the former can be identified as an effective or drift mobility $\left(\mu_{\mathrm{d}}\right)$, i.e.,

$$
\mu_{d}=\mu_{o} \frac{\sqrt{\omega^{2}+1 / \tau_{R}^{2}}}{\sqrt{A^{2}+B^{2}}}
$$

For our photomixing frequency of $252 \mathrm{MHz}, \omega$ is about $1.58 \mathrm{GHz}$ (corresponding to a time scale of $630 \mathrm{ps}$ ). Since the recombination lifetime $\tau_{\mathrm{R}}$ is usually greater than 10 ns, if holds $\omega^{2} \tau_{\mathrm{R}}^{2} \ll 1$, and therefore the above equation can be simplified to

$$
\mu_{d}=\mu_{o} \frac{\omega}{\sqrt{A^{2}+B^{2}}}
$$

From Eqs. (2-13) and (2-16) one can see that the drift mobility $\left(\mu_{\mathrm{d}}\right)$ is an experimentally measurable quantity with no need for any detailed knowledge of trapping and thermal emission. Rewriting Eq.(2-16) to use measurable quantities yields:

$$
\left.\mu_{\mathrm{d}}=\omega \sqrt{\left\langle\sigma_{\mathrm{ac}}^{2}\right.}\right\rangle /\left(\sqrt{2} \mathrm{eG}_{\mathrm{o}} \lambda_{\mathrm{m}}\right)
$$

where $\lambda_{\mathrm{m}}(=7.05 \%)$ is an effective modulation index. Attention is focused on electrons, since their contributions are more dominant to the transport in intrinsic a-Si:H and a$\mathrm{SiC}: \mathrm{H}$ samples than holes.

The root-mean- square ac photoconductivity was determined through the measurement of the power of the ac or photomixing signal using a Tektronix492P spectrum analyzer. The drift mobility $\left(\mu_{\mathrm{d}}\right)$ can thus be obtained from Eq. (2-17) and the lifetime $\tau$ which corresponds to $\mu_{\mathrm{d}}$ can be written as

$$
\tau=\frac{\mu_{o} \tau_{R}}{\mu_{d}}=\frac{\sigma_{d c}}{e G_{o} \mu_{d}}
$$

The photomixing process for single crystalline materials is a special case of the above discussion such that the trapping term in Eq.(2-8) can be set to zero. In this case, the integrals in Eqs.(2-10) and (2-11) are zero and Eqs.(2-17) and (2-18) give back the 
drift mobility $\left(\mu_{d}\right)$ which is equal to the extended state mobility $\left(\mu_{0}\right)$ and the recombination lifetime $\tau_{\mathrm{R}}$ respectively. In the case of amorphous or polycrystalline materials, the drift mobility $\left(\mu_{\mathrm{d}}\right)$ is usually smaller than the extended state mobility $\left(\mu_{\mathrm{o}}\right)$, but approaches $\mu_{\mathrm{o}}$ at high frequencies or in the high temperature limit according to Eqs.(2-10) and (2-11).

The absolute values for the mobility and lifetime can be obtained through the measurement of the absolute values of the microwave photomixing signal and the generation rate.

Specifically, in order to evaluate the temperature dependence of the transport properties under trapping, one can use the following approximations for A and B in Eq. (2-16):

$$
A \approx \frac{1}{\tau_{R}}+\int_{E_{\omega}}^{E_{f n}} K N_{T}(E) d E, \quad B \approx \omega+\frac{\pi}{2} k T K N_{T}\left(E_{\omega}\right),
$$

if $\mathrm{D}>>1$, the frequency-dependent demarcation energy $\mathrm{E}_{\omega}$ is well above the quasi-Fermi level $\mathrm{E}_{\mathrm{fn}}$, which is true in our case. Therefore, and further considering that the recombination effect ( the $1 / \tau_{\mathrm{R}}$ term in the expression for $\mathrm{A}$ ) is negligible:

$$
\begin{aligned}
\mu_{d}=\mu_{o} & \frac{\omega}{\sqrt{\left[\omega+\frac{\pi}{2} k T K N_{T}\left(E_{\omega}\right)\right]^{2}+\left[\varepsilon K N_{T}\left(E_{\omega}\right)\right]^{2}}} \\
& =\mu_{\mathrm{o}} \frac{\omega}{\sqrt{\left[\omega /\left[\mathrm{KN}_{\mathrm{T}}\left(\mathrm{E}_{\omega}\right)\right]+\frac{\pi}{2} \mathrm{kT}\right]^{2}+\left[\varepsilon^{2} \mathrm{Kg}_{\mathrm{c}}\right]}} \times \exp \left(\mathrm{kT} \frac{\ln \left(\mathrm{N}_{\mathrm{c}} \mathrm{K} / \omega\right)}{\varepsilon}\right)
\end{aligned}
$$

where the distribution of tail states is given by $N_{T}=g_{c} \exp (-E / \varepsilon), g_{c}$ is the density of states in the conduction band and $\varepsilon$ is the spread of the band tail.

Thus by measuring the dc and the ac photoconductivities at a single photomixing frequency as a function of temperature, $\mu_{0}, \mathrm{~K}$, and $\varepsilon$ can be determined through curve fitting according to Eq.(2-19). By substitution into Eq.(2-18), the recombination lifetime $\tau_{\mathrm{R}}$ can be calculated. 
It is interesting to point out that when $\varepsilon>\mathrm{kT}$ and the photomixing frequency is much less than a certain "trapping frequency", i.e., $\omega<<(\pi / 2) \operatorname{kTKNT}\left(\mathrm{E}_{\omega}\right)$ and $\omega<<\varepsilon \mathrm{KN}_{\mathrm{T}}\left(\mathrm{E}_{\omega}\right)$, an explicit dispersive transport can be obtained from Eq.(2-19), such that: $\mu_{\mathrm{d}} \propto \omega^{1-\mathrm{kT} / \varepsilon}$.

\subsubsection{Experimental setup for photomixing:}

The block diagram of the experimental setup for the photomixing is shown in Fig. (2-2). The d.c. photosignal was measured by a Keithly 617 programmable Digital Electrometer, and the photomixing signal, i.e., the a.c photosignal, was measured by a Tektronix $492 \mathrm{P}$ spectrum analyzer with a frequency range of $50 \mathrm{KHz}$ to $220 \mathrm{GHz}$. The d.c. bias was supplied by a HP d.c. power supply. The d.c. and a.c. signals were separated by a low pass filter, which are incorporated in a bias tee, that was connected to a three stub tuner. By tuning the stub tuner, the reflection of the photomixing signal from the spectrum analyzer, which is in the microwave range, can be reduced to nearly zero, and thus a true measurement of the photomixing signal can be achieved. All the equipment was controlled by a Labviews program, which works under windows. A Spectra- Physics $125 \mathrm{~A} \mathrm{He}-\mathrm{Ne}$ laser was used as the light source for photomixing [76].

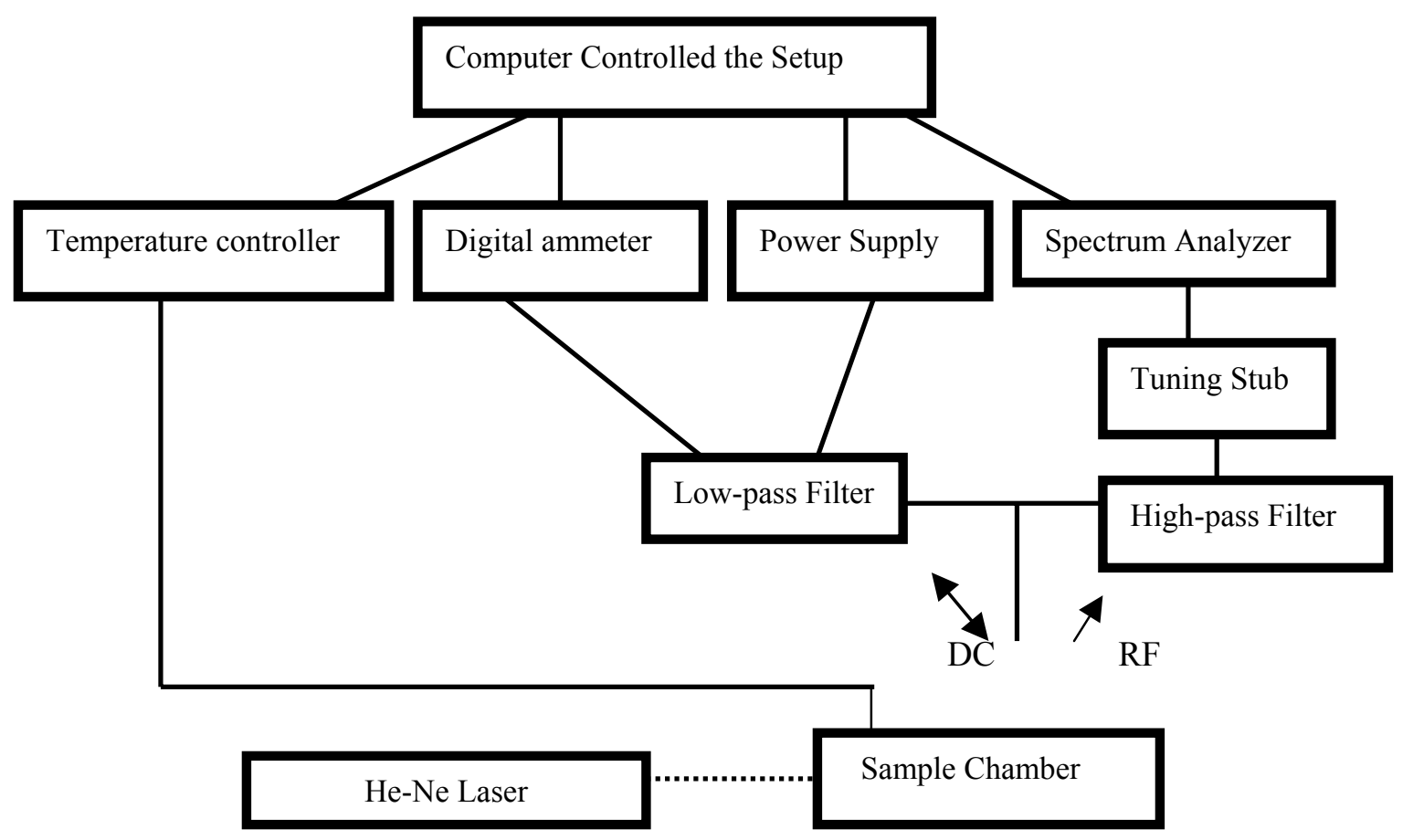

Fig.(2-2) Block diagram of the experimental setup for photomixing. 


\subsubsection{Detection of the microwave photomixing signal:}

\subsubsection{Tuning stub:}

In the steady state limit, i.e., when the wavelength of the electromagnetic signal in the circuit is much larger than the dimension of the circuit, the detection of the electromagnetic signal from a certain source is simple. For example, in order to measure the current $\left(I_{S}\right)$ from the sources (or the sample), one can measure the current $\left(I_{L}\right)$ in the load (or the detector). Thus, in the steady-state limit,

$$
\mathrm{I}_{\mathrm{S}}=\mathrm{I}_{\mathrm{L}}
$$

Or, if one measure the power $\left(\mathrm{P}_{\mathrm{L}}\right)$ obtained by the load (or the detector) with a resistance $\mathrm{R}_{\mathrm{L}}$, one obtains;

$$
\mathrm{I}_{\mathrm{S}}=\mathrm{I}_{\mathrm{L}}=\sqrt{\mathrm{P}_{\mathrm{L}} / \mathrm{R}_{\mathrm{L}}}
$$

For a typical photomixing signal with a frequency of $252 \mathrm{MHz}$, however, since the wavelength is

$$
\lambda=\frac{c}{f}=\frac{3 \times 10^{8} \mathrm{~ms}^{-1}}{252 \times 10^{6} \mathrm{~s}^{-1}}=1.19 \text { meters },
$$

$\lambda$ is comparable to the dimension of the circuit used for the photomixing experiments. Thus the steady-state approximation is not valid: the photomixing signal can be reflected from the load (the spectrum analyzer) and therefore,

$$
\sqrt{\left\langle\mathrm{I}_{\mathrm{S}}^{2}\right\rangle} \neq \sqrt{\left\langle\mathrm{I}_{\mathrm{L}}^{2}\right\rangle}
$$

Employing the three-stub tuner one can eliminate this reflection. The basic idea is that the three-stub tuner can create reflections that can cancel each other through proper tuning. Through the tuning one can achieve:

$$
\mathrm{P}_{\mathrm{Ref}} \approx 0,
$$

where $\mathrm{P}_{\mathrm{Ref}}$ is the power reflected from the three stub tuner. Considering that the tuner, as a non-dissipative device, does not absorb any energy, all the power generated by the sample can be collected by the spectrum analyzer, and then:

$$
\begin{gathered}
\mathrm{P}_{\mathrm{S}} \approx \mathrm{P}_{\text {Mix }}, \\
\text { or } \sqrt{\left\langle\mathrm{I}_{\text {Mix }}^{2}\right\rangle} \approx \sqrt{\mathrm{P}_{\text {Mix }} / \mathrm{R}_{\text {Eff }}},
\end{gathered}
$$


$\mathrm{P}_{\mathrm{Mix}}$ is the average power measured by the spectrum analyzer, $\mathrm{R}_{\mathrm{Eff}}$ is the effective impedance of the three-stub tuner plus that of the spectrum analyzer and $\mathrm{I}_{\mathrm{Mix}}$ is the photomixing current generated in the sample.

\subsubsection{A typical experiment}

Lately, several groups have taken steps into the development and investigation of silicon films in the microcrystalline or $\mu \mathrm{c}-/ \mathrm{a}-\mathrm{Si}: \mathrm{H}$ mixed state. These films typically show relatively high dark currents at room temperature, which in turn limits the maximum electric BIAS which can be applied to rather low values. Accordingly, the accuracy of the photomixing lifetime and mobility determinations suffers from both low electrical dcfields, i.e. low photomixing signals, and, more seriously, from a heating of the sample during the measurements that can lead to an increase of the current of one order of magnitude over the actual photocurrent.

\subsubsection{Improvement of the photomixing instrumentation}

In order to meet the requirements of high accuracy and stable sample temperature, we modified our Photoconductive Frequency Mixing setup to accommodate fields pulses. We are now able to apply BIAS pulses of arbitrary width and frequency. The dc-current is monitored by sampling the voltage over a $1 \mathrm{k} \Omega$ resistor using a PC-DAQ board. The whole setup is controlled using LabVIEW programs [77]. As seen in Fig.(2-3), the computer does not directly switch the sample voltage through the transistor. Instead, it first sends a trigger signal to the function generator, which in turn sends a pulse to the transistor switch. This way we do not actually turn a voltage on and off - instead we periodically connect a power supply to our setup with the voltage being always as high as preset. With a very high impendance sample (like a-Si) the power supply is actually floating while not connected. Connecting such a high voltage with a floating reference to ground necessarily implies certain capacitative discharges (through transformer coupling, e.g.), which occur as current spikes at the raising edge of the pulse. The sharper the edge of the pulse, the higher the spike. So the function generator with it's arbritary waveform feature gives us the chance to apply 'soft' pulses, i.e. pulses with softer edges, to the transistor which leads to significantly lower spikes. The drawback, however, is the time it 
takes the spike to relax - in other words, one usually will have to ignore the first few milliseconds of the pulse. But that is taken care of by the supporting software (see Fig.(24)).

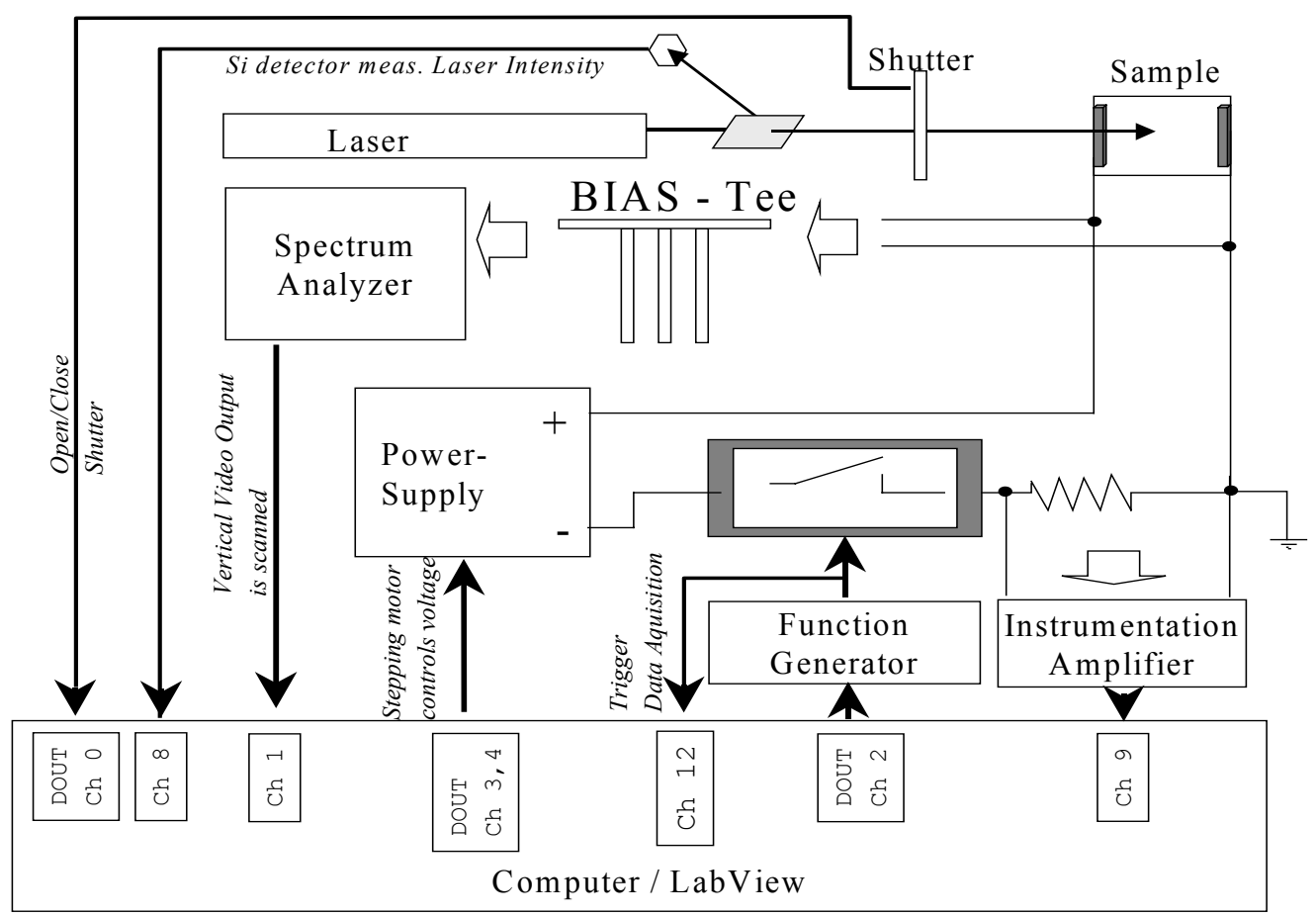

Fig.(2-3) The block diagram of the improved photomixing experimental setup.

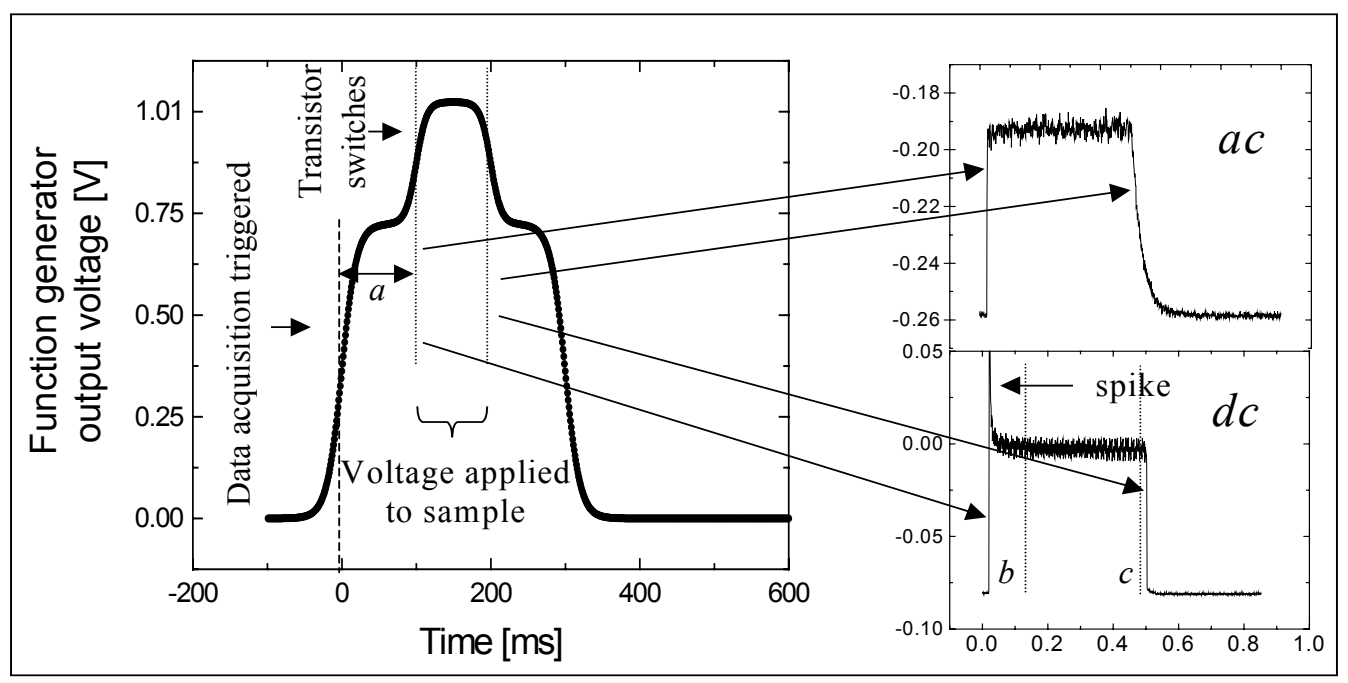

Fig.(2-4) The pulse of the wave generator as a function of time; left, right, the measured ac and dc pulses. 

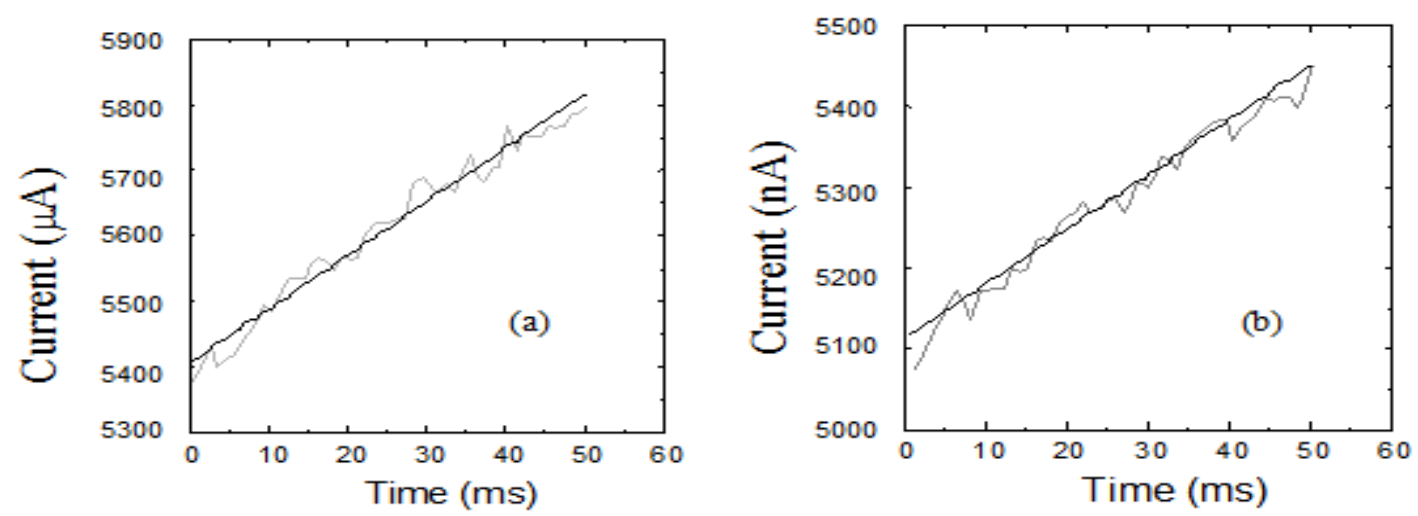

Fig.(2-5) The current through the $\mu \mathrm{c}-\mathrm{Si}$ sample MVS723 (supplied by MVSystems) during one 50ms BIAS pulse under illumination (a) and in dark condition (b). Note the different scales $\mu \mathrm{A}$ versus $\mathrm{nA}$.

Fig.(2-5) shows the time dependent dc-current under BIAS application in the dark and illuminated cases. The sampling rate here is 1000 samples per second (applies for both dc-current and ac-signal), one $50 \mathrm{~ms}$ - pulse is applied per second whereby one measurement cycle consists of one dark and one illuminated signal acquisition. When the photo current is to be measured during a field dependent measurement, the shutter is opened $100 \mathrm{~ms}$ in advance, which means a total illumination duration of 0.25 seconds per two-second-cycle. In the case of decay measurements, the sample is illuminated 1.75 seconds per cycle. These results are then averaged over at least 100 points for each BIAS point in the field-dependent dc- and ac-curves (see Fig. (2-6)).

As can be seen in the Fig. (2-6), given enough data points of the photomixing power to average, we obtain curves with satisfying accuracy whereas the effective power dissipated within the sample is reduced by a factor of 20 as compared to stationary measurements (50ms BIAS vs. 1s per half-cycle). Therefore, electrical fields of much higher magnitude are possible. Also, as shown in Fig.(2-5), the slope in the current due to sample heating during one pulse is well defined so that a linear fit yields the currents at room temperature; in addition, it yields a good measure for the actual temperature increase, which then can be subtracted and/or minimized: i.e. via a preferred BIAS-width / frequency ratio. 
However, the total illumination duration of about 15 minutes during one fielddependent measurement makes degradation corrections necessary, i.e. data points have to be corrected according to the progress of the transport parameters decay and the time they were taken.
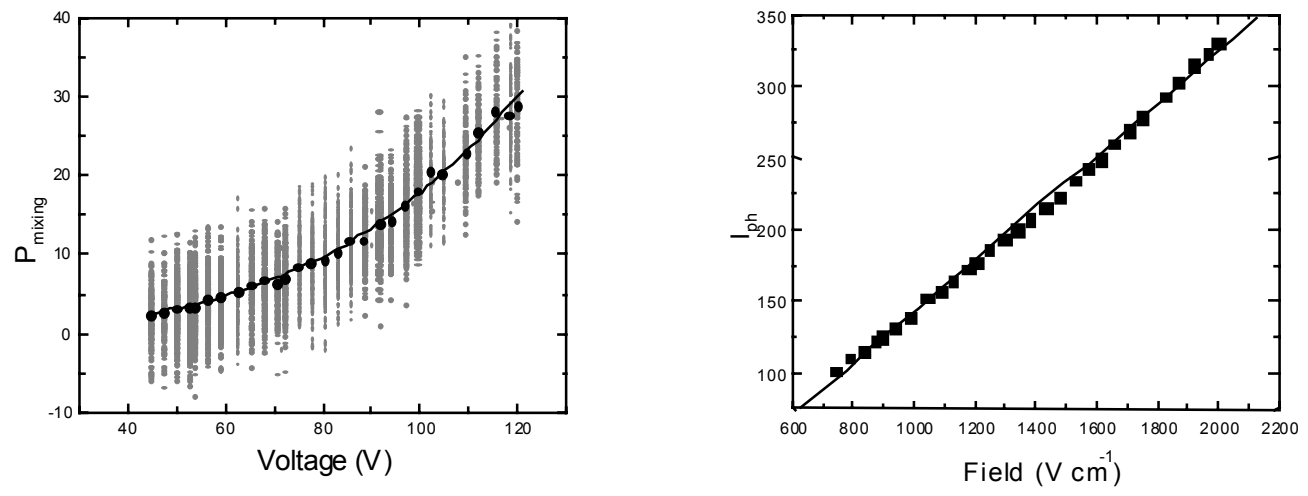

Fig.(2-6) Photomixing power and dc-current signal averaged and fitted (solid curves)(Sample: MVS723, light soaked state).

\subsection{Transient Thermoelectric Effect (TTE)}

Time resolved photo-and thermoelectric effects (TTE) may be used to determine carrier lifetimes, carrier mobilities, the density of trap states and the location of trap levels in crystalline and amorphous semiconductors under basically the same data reduction and are thus in particular useful in the case of mixed amorphous and crystalline states such as the "transitional" epitaxial layers which comprise amorphous regions as well as crystalline ones [78,79].

With the TTE-method, a light pulse, which is limited in space and time, falls on one end of a rectangular sample (here a thin film) and a voltage appears at the end faces, which decays in time. The experimentally observed decay $V(t)$ can be empirically described as a superposition of exponentials :

$$
\mathrm{V}(\mathrm{t})=\mathrm{V}_{\infty}+\sum \mathrm{a}_{\mathrm{i}} \exp \left(-\mathrm{t} / \tau_{\mathrm{i}}\right)
$$

Where the $\mathrm{a}_{\mathrm{i}}, \tau_{\mathrm{i}}$ are the relaxation amplitudes and times of different stages, which are usually connected with different physical phenomena: 


\section{stage1 :}

$\mathrm{V}_{1}(\mathrm{t})$ arises from the Dember effect: charge carriers are generated in pairs in the illuminated region and diffuse into the dark region with unequal mobilities (ambipolar diffusion), whereupon an electrical (Dember-) field appears. The generation rate given, from the decay of this transient, one can derive the lifetime $\tau_{1}$ and from the amplitude a combination of electron and hole-mobilities.

\section{stage2 :}

In a semiconductor without defects, after the light is converted into heat, the illuminated region still has a higher temperature whereupon a secondary ( ambipolar) diffusion process occurs, yielding a socalled "transient thermovoltage". If unintentional (defects) or intentional (doping) trap states exist in the semiconductor, the second transient $V_{2}(t)$ becomes more interesting. Now the internal electrical field arises from the inhomogeneous trapping of carriers, i.e. the space charge centers are differently located because of the ambipolar diffusion of stage 1, which occurred earlier. The decay of $V_{2}(t)$, then depends on the space charge field and the diffusion constant (of the fastest carrier) and thus the initial amplitude $\mathrm{V}_{20}$ and the time constant $\tau_{2}$ are again interdependent. While $\mathrm{V}_{20}$ contains the trap density and level distribution, from the dependence of the time constant $\tau_{2}$ with the temperature one can extrapolate the stage 1 lifetime.

\section{stage 2' :}

While the photoinduced ambipolar diffusion is the primary source for the occurrence of the ambipolar space charge, a secondary space charge source arises from the secondary carrier diffusion, which originates from the (delayed) heating up of the illuminated region (transient Seebeck effect). That transient $\mathrm{V}_{2}{ }^{\prime}(\mathrm{t})$ is again interrelated with its decay time and even more sensitive to the trap (difference) density of states. $\mathrm{V}_{2}{ }^{\prime}$ thus can be best used as a film quality measure.

\section{stage 3 :}

A third transient, $V_{3}(t)$, is connected with the traditional Seebeck-effect, i.e. it originates from the temperature gradient which in the end arises across the whole sample because the light energy is dumped preferentially at the one end. From the decay time of this transient, $\tau_{3}$, one can extract the thermal diffusion constant and from its initial amplitude $\mathrm{V}_{30}$ the thermovoltage. 


\subsubsection{The theory of TTE method:}

Empirically, see Fig.(2-7) the voltage V(t) of stage I and II together can be described by:

$$
\mathrm{V}=\mathrm{V}_{\mathrm{D} 0} \exp \left(-\mathrm{t} / \tau_{1}\right)+\mathrm{V}_{20}\left(1-\exp \left(-\mathrm{t} / \tau_{1^{\prime}}\right)\right) \exp \left(-\mathrm{t} / \tau_{2}\right)
$$

where the first term is time-dependent Dember-voltage $V_{D}(t)$ and $\tau_{1}$ is the relaxation of the ambipolar photodiffusion[80-82]. The initial amplitude $\mathrm{V}_{\mathrm{D} 0}$ is given, for example, in [80], $\mathrm{V}_{20}$ is the initial relaxation amplitude of the ambipolar charge distribution, $\tau_{1}$, characterizes its build-up, and $\tau_{2}$ its decay Fig.(2-7). From Eq.(2-28), one could formally extract three time constants, $\tau_{1}, \tau_{2}$ and $\tau_{1}$, but they would be correlated. If stage 1 is very fast as compared to the time resolution of the set-up, one measures $\tau_{2}$ only.

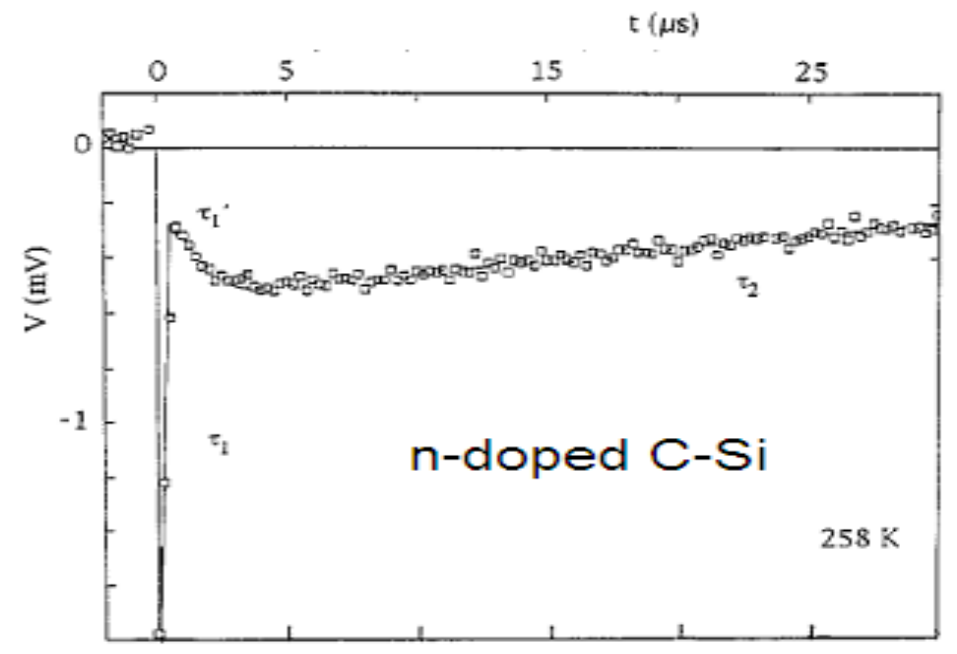

Fig.(2-7)The transient voltage of the n-doped c-Si sample versus the time scale at $258 \mathrm{~K}$.

\subsubsection{Ambipolar charge distribution:}

We have seen that when the ambipoar diffusion is finished, another process, which gives rise to an internal electric field, takes over. This field is thought to be connected with a spatial imbalance of the trap state occupation: during ambipolar diffusion, the excess electrons and holes not only move spatially separated but also the electrons and holes usually have different capture and reemission coefficients with the gap states and thus in general a trap charge imbalance, i.e. an ambipolar charge distribution, will occur. The simplest case would be to assume trapping of one species, say holes, while the electrons are immediately reemitted. This would result in an electron 
excess peak which is pulled back by the fixed positive charges, which are left behind. That scenario provides the base for the calculation of the stage 2 transient.

\subsubsection{Calculation of the stage $\mathrm{II}_{1}$ relaxation time $\tau_{2}$ :}

Generally, the diffusion equation connects any time regime with a spatial regime. Thus, if the stage 1 excitation starts at $\mathrm{x}=0$ and $\mathrm{t}=0$, when this process is practically finished- after $\mathrm{t}=\tau_{1}-\mathrm{a}$ (dark) portion $\mathrm{L}_{1}=\sqrt{\mathrm{D}_{\mathrm{n}} \tau_{1}}$ of the sample is involved and $\mathrm{a}$ decreasing internal electric field $\mathrm{E}_{\mathrm{i}}$ will exist in the region $0<\mathrm{x}<\mathrm{L}_{1}[81,84)$. For stage II, the decay of the remaining excess electron density $\delta$ n will take place predominantly in spatial region $\mathrm{L}_{1}<\mathrm{x}<\mathrm{L}_{2}$ and during $\tau_{1}<\mathrm{t}<\tau_{2}$. The excess carrier density $\delta \mathrm{n}(\mathrm{x}, \mathrm{t})$ is then given by a transport equation which involves field, diffusion and recombination terms. Using the differential equation from [85] but with a recombination term, $\partial \mathrm{n} / \tau$, added, we have

$$
\partial \delta \mathrm{n}(\mathrm{x}, \mathrm{t}) / \partial \mathrm{t}=\mu_{\mathrm{n}}\left(\mathrm{E}_{\mathrm{i}}(\mathrm{t}) \partial \delta \mathrm{n} / \partial \mathrm{x}+\mathrm{n}_{0} \partial \mathrm{E}_{\mathrm{i}} / \partial \mathrm{x}\right)+\mathrm{D}_{\mathrm{n}} \partial^{2} \delta \mathrm{n} / \partial \mathrm{x}^{2}-\delta \mathrm{n} / \tau
$$

When we assume an almost homogeneous electric field, $\delta \mathrm{E}_{\mathrm{i}} / \delta \mathrm{x} \approx 0$ (like in $\mathrm{c}-\mathrm{Si}$ ), or an initial carrier concentration $\mathrm{n}_{0}$ which is small in comparison with the photogenerated carrier excess, $\mathrm{n}_{0} \ll \delta$ n (this is usually the case with a-Si:H), we may always neglect the second term in the bracket. For a-Si, with a spread in the trap level energies, $\tau(\mathrm{E})$, and $\tau$ would represent a center of gravity.

The origin of the $\mathrm{x}$-coordinate is renormalized (now at $\mathrm{L}_{1}$ ) and the initial excess carrier concentration $\delta \mathrm{n}_{0}$ is that which could not recombine and is left over from the initial excitation after $\tau_{1}$.

Then, for a solution of Eq.(2-29) we try ( $\mathrm{L}_{2}$ :diffusion length):

$$
\begin{aligned}
& \delta \mathrm{n}(\mathrm{x}, \mathrm{t})=\delta \mathrm{n}_{0} \cdot \mathrm{F}(\mathrm{t}) \cdot \exp \left(-\mathrm{x} / \mathrm{L}_{2}\right) \quad \text { for } \mathrm{x}>0 \\
& \delta \mathrm{n}(\mathrm{x}, \mathrm{t})=0 \text { for } \mathrm{x}<0
\end{aligned}
$$

together with an opposite surface charge at $\mathrm{x}=-\mathrm{L}_{1}$. For $\mathrm{F}(\mathrm{t})$ one then obtains the differential equation (Eqs.(2-29) and (2-30)):

$$
(1 / F) \partial F / \partial t=-\mu_{n} E_{i}(t) / L_{2}+D_{n} / L_{2}^{2}-1 / \tau
$$


whose solution requires an $\mathrm{E}_{\mathrm{i}}(\mathrm{t})$ which is consistent with Poissons equation. Assuming the internal field to decay exponentially $\mathrm{E}_{\mathrm{i}}(\mathrm{t})=\mathrm{E}_{0} \exp \left(-\mathrm{t} / \tau_{2}\right)$, we obtain:

$$
\begin{aligned}
\mathrm{F}(\mathrm{t})= & \exp \left[\left(-\mu_{\mathrm{n}} \tau_{2} \mathrm{E}_{0}\left(1-\exp \left(-\mathrm{t} / \tau_{2}\right)\right) / \mathrm{L}_{2}\right)+\left(\mathrm{D}_{\mathrm{n}} / \mathrm{L}_{2}^{2}-1 / \tau\right) \mathrm{t}\right] \\
& \approx \exp -\left(\mu_{\mathrm{n}} \mathrm{E}_{0} / \mathrm{L}_{2}-\mathrm{D}_{\mathrm{n}} / \mathrm{L}_{2}^{2}+1 / \tau\right) \mathrm{t}
\end{aligned}
$$

As $F(t)$ describes the decay of the excess carrier concentration, its characteristic decay time should be equal to that of the electric field, at least for small t. Expanding $\exp \left(-t / \tau_{2}\right)$ in the exponent, we indeed obtain an exponential decay. Setting this decay time equal to $\tau_{2}$ and $L_{2}=\sqrt{D_{n} \tau_{2}}$ one obtains:

$$
2 / \tau_{2}=1 / \tau+\mu_{\mathrm{n}} \mathrm{E}_{0} / \sqrt{\mathrm{D}_{\mathrm{n}} \tau_{2}}
$$

For large electrical fields $\mathrm{E}_{0}=\mathrm{V}_{0} / \mathrm{L}_{1}$ with $\mathrm{L}_{1}=\sqrt{\mathrm{D}_{\mathrm{n}} \tau_{1}}$ and/or large time constants $\tau$, Eq.(2-33) reduces to $1 / \tau_{2} \approx\left(\mu_{\mathrm{n}} \mathrm{E}_{0}\right)^{2} / 4 \mathrm{D}_{\mathrm{n}}=\left(\mathrm{eV}_{0} / 2 \mathrm{kT}\right)^{2} \tau_{1}$; in this case the charge redistribution is only due to carrier drift and diffusion; this can be deduced from the fact that this result is also obtained by setting the diffusion length $L_{2}=\sqrt{D_{n} \tau_{2}}$ equal to the drift distance $L_{D}=V_{D} \tau_{2}$ or that one obtains the same result by solving the differential Eq.(2-29) without the recombination term [83]. Also, in that case, $\tau_{2}$ becomes independent of the sign of $\mathrm{E}_{0}$ and the mobility cannot be obtained.

If $1 / \tau$ compares with the field dependent term Eq.(2-33), the sign of $\mathrm{E}_{0}$ becomes important. Like with ambipolar diffusion the field always acts opposite to the diffusion direction, i.e. $+x$ and thus $E=-\left|E_{0}\right|$. Then, Eq.(2-33) becomes:

$$
2 / \tau_{2} \approx 1 / \tau-\mu_{\mathrm{n}}\left|\mathrm{E}_{0}\right| / \sqrt{\mathrm{D}_{\mathrm{n}} \tau_{2}} \approx 1 / \tau-\left(\mathrm{eV}_{0} / \mathrm{kT}\right) / \sqrt{\tau_{1} \tau_{2}}
$$

According to Eq.(2-34), $\tau_{2}>\tau$, as is observed. However, $\tau_{2}$ depends on the magnitude of the (initial) electric field, i.e. on the amplitude of this relaxation stage. For small electric fields and/or high temperatures, we still obtain $\tau_{2}>\tau$, but the field dependence disappears: in this limit $2 / \tau_{2} \approx 1 / \tau$. In that regime, the temperature and doping dependence of stage 2 and 1 should be identical. As $\tau_{2}>\tau>\tau_{1}, \tau$ cannot be smaller as $\tau_{1}$, if we let $\tau=\tau_{1}$, Eq.(2-34) can be solved explicitly to yield $\tau_{2}(\mathrm{~T})$ :

$$
1 / \tau_{2}=\left(1 / 2 \tau_{1}\right)\left\{1+\mathrm{y}^{2}-\mathrm{y} \sqrt{2+\mathrm{y}^{2}}\right\} ; \quad \mathrm{y}=\mathrm{eV}_{0} / 2 \mathrm{kT}
$$




\subsubsection{Calculation of the relaxation amplitude $V_{0}$ of stage $I_{1}$ :}

For the hole concentration we may use a solution similar to Eq.(2-30):

$$
\begin{array}{lc}
\delta \mathrm{p}=\delta \mathrm{p}_{0} \exp \left(-\mathrm{y} / \mathrm{L}_{2 \mathrm{p}}\right) \exp \left(-\mathrm{t} / \tau_{2 \mathrm{p}}\right) & \text { for } \mathrm{y}>0 \\
\delta \mathrm{p}(\mathrm{x}, \mathrm{t})=0 \text { for } \mathrm{y}<0 ; & \mathrm{y}=-\left(\mathrm{x}+\mathrm{L}_{1}\right)
\end{array}
$$

where $L_{2 p}=\sqrt{\tau_{2 p} D_{p}}$ is small even against $L_{1}$, because the diffusion constant of the holes $D_{p}$ is assumed to be greatly reduced ( trapping of holes). In that case the total (surface) charge $\mathrm{eN}^{+}$at $\mathrm{x}=-\mathrm{L}_{1}$ as obtained by integration would be:

$$
\mathrm{N}^{+}(\mathrm{t})=\int_{0}^{\infty} \delta \mathrm{p}(\mathrm{x}, \mathrm{t}) \mathrm{dx}=\delta \mathrm{p}_{0} \mathrm{~L}_{2 \mathrm{p}} \exp \left(-\mathrm{t} / \tau_{2 \mathrm{p}}\right)=\mathrm{P}_{0+} \exp \left(-\mathrm{t} / \tau_{2 \mathrm{p}}\right)
$$

and can be set equal to decaying positive surface charge $\mathrm{eP}_{0+}$. The equivalent negative surface charge $\mathrm{eN}^{-}$at $\mathrm{x}=0$ is:

$$
\mathrm{N}^{-}(\mathrm{t})=\int_{0}^{\infty} \delta \mathrm{n}(\mathrm{x}, \mathrm{t}) \mathrm{dx}=\delta \mathrm{n}_{0} \mathrm{~L}_{2 \mathrm{n}} \exp \left(-\mathrm{t} / \tau_{2 \mathrm{n}}\right)=\mathrm{P}_{0-} \exp \left(-\mathrm{t} / \tau_{2 \mathrm{n}}\right)
$$

Because of charge neutrality, we should have $P_{0-}=P_{0+}$ and $\tau_{2 n}=\tau_{2 p}=\tau_{2}$. We also have a capacitor geometry and this would be consistent with the assumption of homogeneity for $\mathrm{E}_{\mathrm{i}}(\mathrm{t})$. Also, we can recover the time dependence of the internal electrical field through $\varepsilon \mathrm{E}=\mathrm{D}=\sigma=\mathrm{eN}^{-}$. This is equivalent to the use of Poissons equation. One obtains:( $\varepsilon$ is the dielectric constant)

$$
\mathrm{E}_{\mathrm{i}}(\mathrm{t})=\mathrm{E}_{0} \exp \left(-\mathrm{t} / \tau_{2}\right) \quad \text { where e } \delta \mathrm{p}_{0} \mathrm{~L}_{2 \mathrm{p}} / \varepsilon=\mathrm{E}_{0}=\mathrm{e} \delta \mathrm{n}_{0} \mathrm{~L}_{2 \mathrm{n}} / \varepsilon
$$

consistent with the initial assumption. As $\mathrm{E}_{0}$ exists after $\tau_{1}$ inside $0<\mathrm{x}<\mathrm{L}_{1}$, a numerical estimate is $\mathrm{E}_{0} \approx \mathrm{V}_{0} / \mathrm{L}_{1}$. In order to calculate $\mathrm{V}_{0}(\mathrm{t}), \delta \mathrm{n}_{0}$ or $\delta \mathrm{p}_{0}$ have to be known, they are not constant with the temperature like with photoexcitation, because the capture and reemission from trap states are important here and are temperature dependent. In particular, we assume that the excess carrier concentration is related to the difference in capture and reemission like with the excess recombination given in [80]:

$$
\delta \mathrm{n}_{0} / \tau=\mathrm{C}_{\mathrm{n}}\left[\mathrm{n}(1-\mathrm{f})-\mathrm{fg}^{-1} \mathrm{~N}_{\mathrm{c}} \exp \left(-\Delta \varepsilon^{+} / \mathrm{kT}\right)\right] ; \mathrm{C}_{\mathrm{n}}=\mathrm{N}_{\mathrm{T}} \mathrm{v}_{\mathrm{th}} \sigma\left(\mathrm{D}^{+}\right)
$$

here $\mathrm{f}$ is the Fermi function, $\mathrm{g}^{-1}$ is the spin factor, $\mathrm{N}_{\mathrm{T}}$ is the electron trap density, $v_{\text {th }}$ is the thermal velocity, $\sigma$ is the donor $\mathrm{D}^{+}$capture cross section, $\mathrm{N}_{\mathrm{c}}$ is the conduction band 
density of states, and $\Delta \varepsilon^{+}$is the $\mathrm{D}^{+}$trap level below the conduction band. This means that the initial amplitude of stage 2 transient contains information about the trap levels and their distribution, but also $\tau_{2}$ contains that information because of its field dependence Eq. (2-35).

For highly doped samples, the chemical potential $\mu$ always stays fairly close to the trap level $\mathrm{E}_{\mathrm{T}}$, i.e. $\mathrm{E}_{\mathrm{T}}-\mu=\Delta \varepsilon^{\prime}$ and $\mathrm{f} \approx(1 / 2)\left(1-\Delta \varepsilon^{\prime} / \mathrm{kT}\right)$, which yields the following approximation for $\delta \mathrm{n}_{0}$ and with it for $\mathrm{V}_{0}(\mathrm{~T})$ :

$\mathrm{V}_{0+} \approx\left(\tau_{1} \mathrm{eL}_{1} \mathrm{~L}_{2 \mathrm{n}} / 2 \varepsilon\right) \mathrm{nC}_{\mathrm{n}} \mathrm{H}(\mathrm{T})$

$\mathrm{H}(\mathrm{T})=\left[1-\mathrm{c}_{1+} \exp \left(-\Delta \varepsilon^{+} / \mathrm{kT}\right)+\left(\Delta \varepsilon^{+^{\prime}} / \mathrm{kT}\right)\left(1+\mathrm{c}_{1+} \exp \left(-\Delta \varepsilon^{+} / \mathrm{kT}\right)\right)\right]$

$\mathrm{c}_{1+}=\mathrm{g}^{-1} \mathrm{~N}_{\mathrm{c}} / \mathrm{n}>1$; which suggests a slow increase of the TTE-voltage at low temperatures and a nearly exponential drop at higher temperatures because of an increasing reemission. Such a behavior can indeed be observed in Fig.(2-8) for n-doped c-Si.

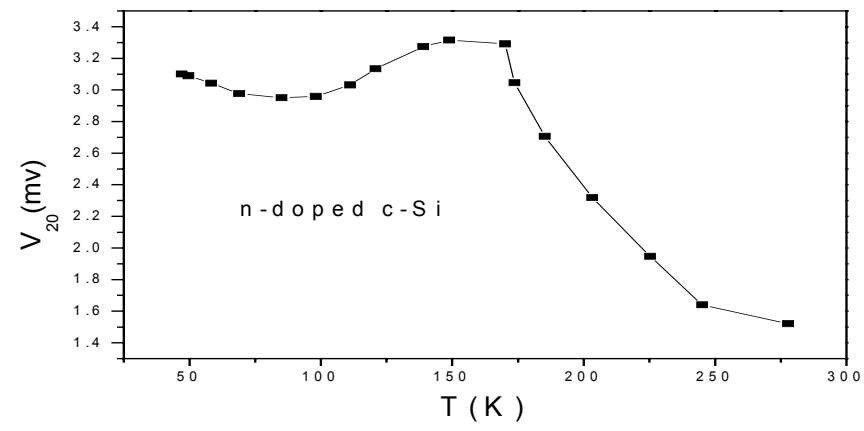

Fig.(2-8) The transient voltage $\mathrm{V}_{20}$ of the n-doped c-Si sample versus the temperature.

If we have traps for both electrons and holes, as additional but oppositely polarized voltage $\mathrm{V}_{0 \text { - }}$ appears which has the same structure as Eq.(2-41) but with the parameters related to the valence band edge, and:

$$
\mathrm{V}_{0}=\mathrm{V}_{0-}+\mathrm{V}_{0+}
$$

If there is a distribution of trap levels such as in amorphous materials, one should use a weighted integration over all gap states, i.e. doping levels and states from structural disorder: 


$$
\mathrm{V}_{0}=\left(2 / \mathrm{E}_{\mathrm{g}}\right) \int_{0}^{\mathrm{E}_{\mathrm{a}} / 2} \mathrm{~V}_{0-} \mathrm{dE}-\left(2 / \mathrm{E}_{\mathrm{g}}\right) \int_{\mathrm{E}_{0} / 2}^{\mathrm{E}_{\mathrm{g}}} \mathrm{V}_{0+} \mathrm{dE}
$$

According to Eq.(2-40) and Eq.(2-43), $\mathrm{V}_{0}(\mathrm{~T})$ does contain information about the trap state difference distribution $\mathrm{N}_{\mathrm{T}}^{+}(\mathrm{E})-\mathrm{N}_{\mathrm{T}}^{-}(\mathrm{E})$, which reduces to a single carrier trap density $\mathrm{N}_{\mathrm{T}}$ only if there are no levels for the other kind of carrier.

\subsubsection{Transient Seebeck-effect:}

As pointed out by $[83,84]$, secondary excess carriers, which are thermally generated through local heating of the illuminated section should also diffuse away, and thus also contribute to the ambipolar charge distribution. If the same redistribution mechanisms are active for this contribution, it would simply add to the photogenerated field for $\mathrm{t} \ll \tau_{2}$, but in contrast to that it does not vanish for $\mathrm{t} \gg \tau_{2}$; in this limit it assumes a small steady state value $\mathrm{E}_{\mathrm{s}}$, assuming that the temperature profile still exists. Thus, for the total internal field to assume $E_{0}$ for $t \ll \tau_{2}$ and $E_{s}$ for $t \gg \tau_{2}$ we may follow [83] and write:

$$
E_{i}(t)=E_{0} \exp \left(-t / \tau_{2}\right)+E_{s}\left(1-\exp \left(-t / \tau_{2}\right)\right)
$$

The arrangement of terms shows that one has implicitly assumed that the secondary effect increases as the primary effect decreases, using the same time constant. Then, the observed voltage $\mathrm{V}(\mathrm{t})$ is obtained from Eq.(2-44) by integration over the sample length [83]. Eq.(2-44), however, does not describe the decay of $E_{s}$. $E_{s}$ should go to zero when

the temperature becomes constant over the diffusion length of the electrons or holes, $\mathrm{L}_{2 \mathrm{n}, \mathrm{p}}$, and thus a better empirical equation should be:

$$
\mathrm{V}(\mathrm{t}) \approx \mathrm{V}_{0} \exp \left(-\mathrm{t} / \tau_{2}\right)+\mathrm{V}_{\mathrm{s}}\left(1-\exp \left(-\mathrm{t} / \tau_{2}\right)\right) \exp \left(-\mathrm{t} / \tau_{2^{\prime}}\right)
$$

Indeed, Eq.(2-45) fits the experiments better that Eq.(2-44).

\subsubsection{Calculation of the relaxation time $\tau_{2}$,}

In order to calculate $\tau_{2}$, , one may start from a $\delta$-function-like temperature peak at $\mathrm{x}=0=\mathrm{t}$; then, for finite times we would obtain a Gaussian temperature distribution: $\delta \mathrm{T}(\mathrm{x}, \mathrm{t})=\delta \mathrm{T}_{0}\left(4 \mathrm{D}_{\mathrm{T}} \mathrm{t}\right)^{-1 / 2} \exp \left(-\mathrm{x}^{2} / 4 \mathrm{D}_{\mathrm{T}} \mathrm{t}\right)$. Asking for a drop in temperature to say, $10 \%$ percent over a diffusion length $\mathrm{L}_{2}$, for the characteristic time $\tau_{2}$, we would obtain: 
$1 / \tau_{2}, \approx \alpha \mathrm{D}_{\mathrm{T}} / \mathrm{L}_{2}^{2}$ or $\tau_{2} / \tau_{2},=\tau_{\mathrm{D}} / 4 \tau_{3}$

where the characteristic times are defined as:

$$
\tau_{\mathrm{D}}=\alpha \mathrm{L}^{2} / \mathrm{D}^{*} ; \tau_{3}=\mathrm{L}^{2} / 4 \mathrm{D}_{\mathrm{T}} ; \quad \tau_{2}=\mathrm{L}_{2}^{2} / \mathrm{D}^{*} ; \mathrm{D}^{*}=\mathrm{D}_{\mathrm{n}, \mathrm{p}}(\mathrm{T}) ; \quad \alpha=\alpha_{\mathrm{n}, \mathrm{p}}
$$

$0<\alpha<1$ is a factor which represents the chosen temperature decay cut-off, while $\mathrm{L}_{2}=$

$\mathrm{L}_{2 \mathrm{n}, \mathrm{p}}$, depending on whether $\mathrm{E}_{\mathrm{s}}$ is governed by electrons or holes. Eq.(2-46) allows to calculate $\tau_{2}$, from the thermal diffusivity $\mathrm{D}_{\mathrm{T}}$ and the carrier mobility, once $\alpha$ is known.

\subsubsection{Calculation of the relaxation amplitude $V_{s}$ :}

While electrical equilibrium is found locally after $\tau_{2}$, because of the temperature gradient $T_{2}-T_{1}$ over the length $L_{2}$, the chemical potential $\mu(T)$ has a gradient, too $[86,87]$. Taking for simplicity the capture only at $T_{1}$ and the remission only at $T_{2}$, the bracket in Eq.(2-41) reduces to the $\varepsilon_{0} / \mathrm{kT}$ term, with $\varepsilon_{0}=\Delta \varepsilon_{2},-\Delta \varepsilon_{1}$. Then, for $\mathrm{V}_{\mathrm{s}}(\mathrm{T})$ one obtains:

$$
\begin{aligned}
& \mathrm{V}_{\mathrm{s}+} \approx\left(\tau_{2} \mathrm{eL}_{2 \mathrm{n}} \mathrm{L}_{2 \mathrm{n}^{\prime}} / 2 \varepsilon\right) \mathrm{nC}_{\mathrm{n}} \mathrm{G}(\mathrm{T})=\mathrm{A}^{+} \cdot \mathrm{G}(\mathrm{T}) \\
& \mathrm{G}(\mathrm{T})=\left(\varepsilon_{0+} / \mathrm{kT}\right)\left(1+\mathrm{c}_{1+} \exp \left(-\Delta \varepsilon^{+} / \mathrm{kT}\right)\right) \\
& \text { and } \quad \mathrm{V}_{\mathrm{s}}=\mathrm{V}_{\mathrm{s}}+\mathrm{V}_{\mathrm{s}^{+}}
\end{aligned}
$$

if traps exist for both electrons and holes. For amorphous semiconductors again one has to use a weighted integration equivalent to Eq.(2-43). However, since this $G(T)$ term has a maximum at $\Delta \varepsilon^{+}=\mathrm{kT}$, it has a scanning function, i.e. with increasing temperature $\mathrm{V}_{2+}$, it probes deeper into the gap $\mathrm{E}_{\mathrm{g}}=\mathrm{E}_{\mathrm{c}}-\mathrm{E}_{\mathrm{v}}$ giving values for the trap difference density of states $\mathrm{N}_{T}^{-}-\mathrm{N}_{\text {T. }}^{+}$. This has been demonstrated for an n-doped c-Si sample Fig.(2-9). According to Eq.(2-41) $\mathrm{V}_{2}$ has this quality to a far lesser extent as $\mathrm{H}(\mathrm{T})$ does not have that scanning function. However, $\mathrm{V}_{2}$ in principle contains the same information as $\mathrm{V}_{\mathrm{s}}$. $\operatorname{In}[88]$, the authors have used $2 \mathrm{kT}$ as an integration cut-off. This will shift the true energy scale somewhat. We can, however, use the phosphor level of our n-doped c-Si sample for a recalibration. 


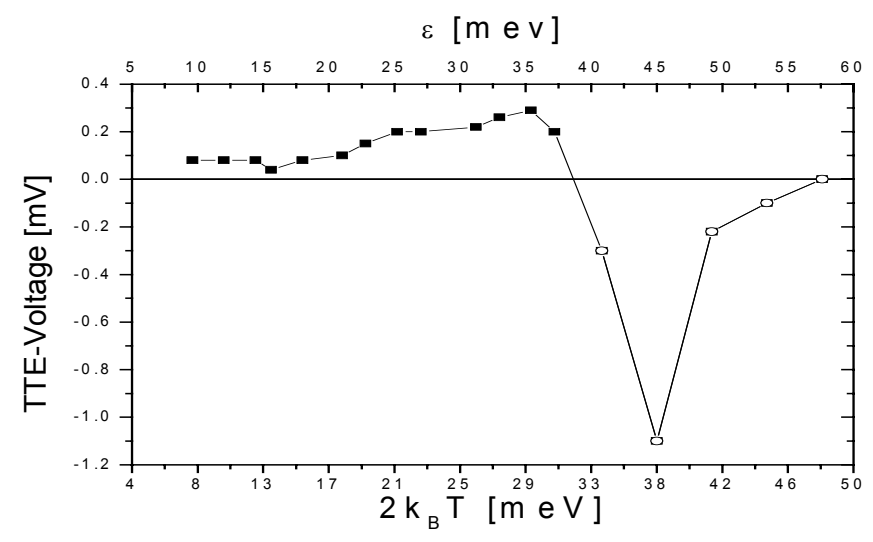

Fig.(2-9) The transient voltage $\mathrm{V}_{\mathrm{s}}$ of the $\mathrm{n}$-doped c-Si sample versus $2 \mathrm{k}_{\mathrm{B}} \mathrm{T}$. The doping level of phosphorus is $45 \mathrm{meV}$ according to [86] (see upper scale).

\subsubsection{Trap density of state as extracted for $\mathrm{V}_{2}$ :}

According to Eq.(2-41), for a single donor level $\mathrm{D}^{+}$the space charge voltage $\mathrm{V}_{2}$ is,

$$
\mathrm{V}_{2}^{+}=\mathrm{A}_{\mathrm{o}}^{+} \mathrm{C}_{\mathrm{n}} \mathrm{H}(\mathrm{T}), \quad \mathrm{C}_{\mathrm{n}}=\mathrm{N}_{\mathrm{T}} v_{\text {th }} \sigma\left(\mathrm{D}^{+}\right)
$$

If we rewrite to emphasize the trap density of states

$$
\mathrm{V}_{2}^{+}=\mathrm{A}^{+} \mathrm{N}_{\mathrm{T}} \mathrm{H}(\mathrm{T}), \quad \mathrm{A}^{+}=\mathrm{A}_{0}^{+} \mathrm{v}_{\text {th }} \sigma
$$

If there is a distribution of donor levels, one has to sum up over all individual levels, which can contribute to the space charge:

$$
\left\langle\mathrm{V}_{2}^{+}\right\rangle=\int_{0}^{\mathrm{E}_{\varepsilon}^{1} / 2} \mathrm{~A}^{+} \mathrm{H}(\mathrm{T}) \mathrm{N}_{\mathrm{T}}(\varepsilon) \mathrm{d} \varepsilon,
$$

where $\varepsilon$ is the energy distance from the conduction band into the gap.

If one goes back to a single level at $\varepsilon_{0}, \mathrm{~N}_{\mathrm{T}}(\varepsilon) \sim \delta\left(\varepsilon-\varepsilon_{0}\right)$. Then, from Eq.(2-50) one recover Eq. $(2-49 b)$.

If one defines a weighted density of state function,

$$
\mathrm{N}_{\mathrm{T}}^{*+}=\mathrm{A}^{+} \mathrm{H}(\mathrm{T}) \mathrm{N}_{\mathrm{T}}(\varepsilon)
$$

from the differential of the measured voltage one can obtain the difference weighted density of state function, which should be close to the true difference density of state as $\mathrm{H}(\mathrm{T})$ is a continuous function.

$$
\frac{\mathrm{d}}{\mathrm{dT}}\left\langle\mathrm{V}_{2}\right\rangle=\frac{\mathrm{d}}{\mathrm{dT}}\left\langle\mathrm{V}_{2}^{+}\right\rangle-\frac{\mathrm{d}}{\mathrm{dT}}\left\langle\mathrm{V}_{2}^{-}\right\rangle=\mathrm{N}_{\mathrm{T}^{+}}^{*}-\mathrm{N}_{\mathrm{T}^{*}}^{*-}
$$


If one wants to plot $\mathrm{N}_{\mathrm{T}}^{+}-\mathrm{N}_{\mathrm{T}}^{-}$versus $\varepsilon$ one has to convert the temperature into $\varepsilon$, i.e. $\varepsilon=\alpha \mathrm{kT}$ : the factor $\alpha$ can be set equal to 2 as with the integration of $\mathrm{V}_{\mathrm{s}}$ or one can determine $\alpha$ by using a known doping level.

Fig.(2-10) shows $d<V_{2}>/ d T$ for phosphor doped crystalline Si sample with $\varepsilon(\mathrm{P})=45$ $\mathrm{meV}$, which would give $\alpha=2.73$.

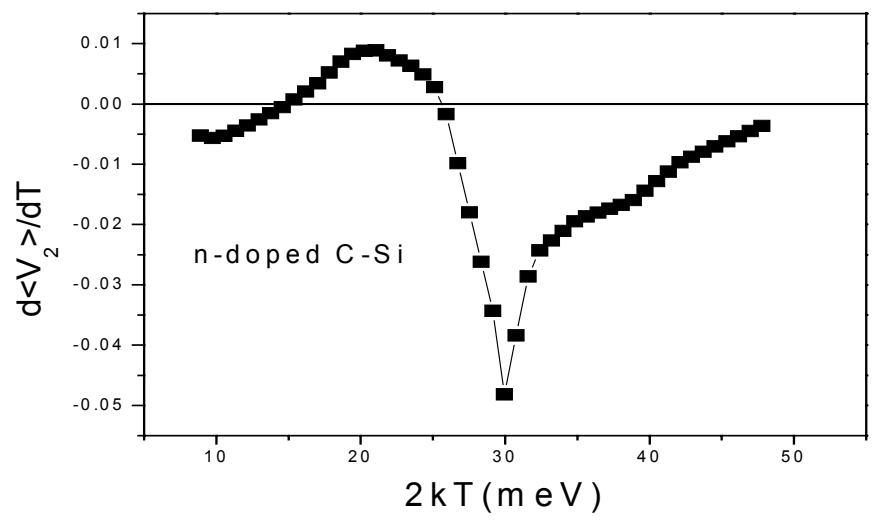

Fig.(2-10) $d<V_{2}>/ d T$ of the $n$-doped c-Si sample versus $2 k T$.

Note that while the structures derived from $V_{2}$ and $V_{s}$ are indeed similar, the difference density of state peaks as extracted from $V_{2}$ is in general broader than those obtained from $\mathrm{V}_{\mathrm{s}}$ and the recalibration factor is larger. That probably results from the convolution with $\mathrm{H}(\mathrm{T})$, which also tends to over-emphasize the density of states at lower temperatures. Note, however, that always $V_{s} \ll V_{2}$ so that very often one has to resort to $V_{2}$, in particular if the small $\mathrm{V}_{\mathrm{s}}$ cannot be separated from the composite signal.

\subsubsection{Experimental Setup for TTE:}

The block diagram of the experimental setup for the transient thermoelectric effects (TTE) is shown in Fig. (2-11). The light pulse, which is limited in space and time, is applied close to one of the long ends of a thin rectangular sample (flash method). The contact space is $\sim 0.5 \mathrm{~mm}$ and the decaying voltage is measured between them [83]; More details of the data reduction are given in $[88,90]$. 


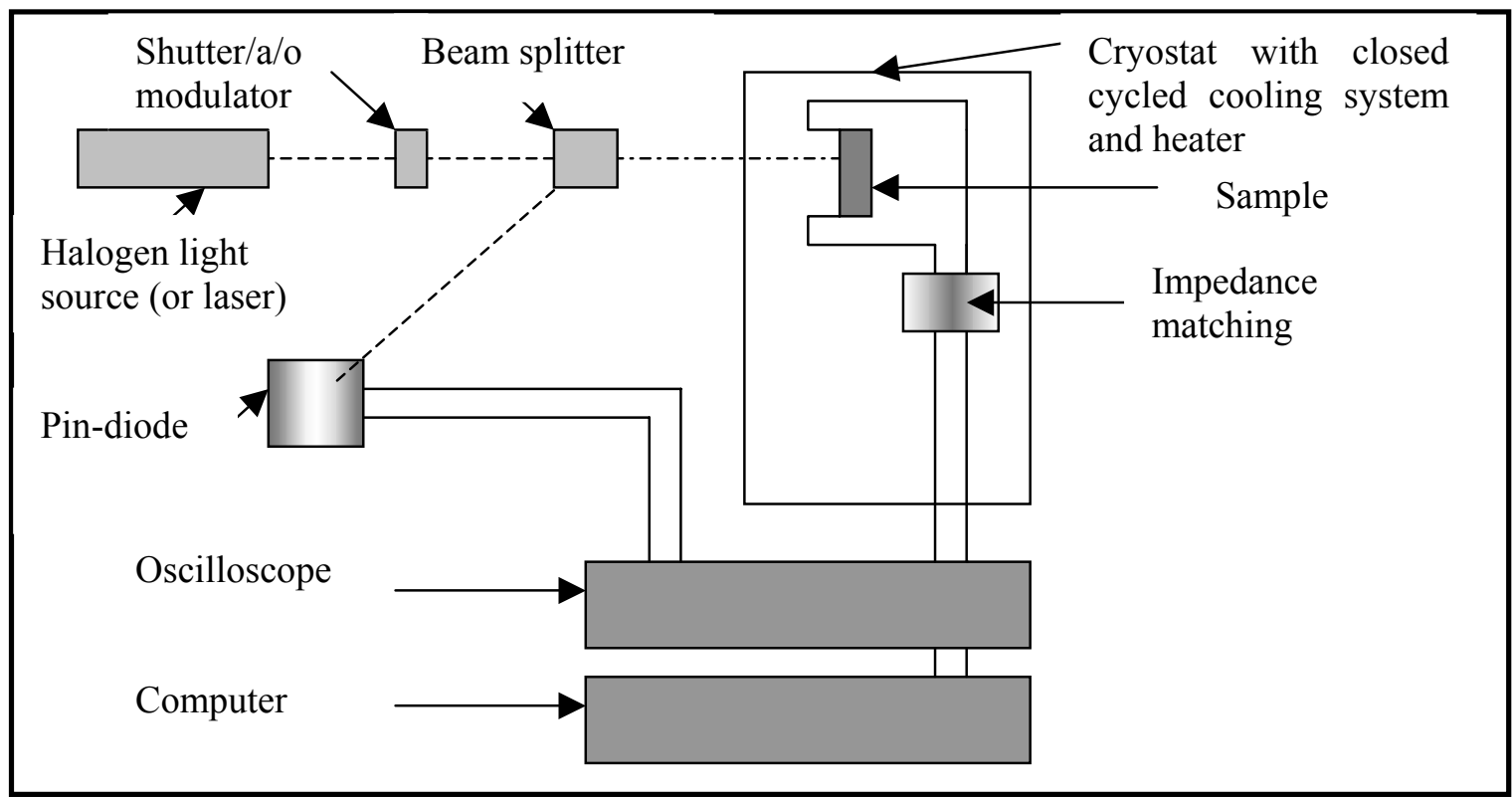

Fig.(2-11) The block diagram of the transient thermoelectric effect (TTE) signals.

\subsubsection{Extraction of $V_{2}(t)$ :}

We did not measure the stages 1 and 3 , as $\mathrm{V}_{1}, \tau_{1}$ is given by photomixing and as $\mathrm{V}_{3}, \tau_{3}$ does not directly relate to the electronic characterization. However, stage 2 could be readily obtained; however, for these samples $V_{2}^{\prime}(t)$ could not be discriminated from $V_{2}(t)$. Empirically, that separation works well only if the signs of $V_{2}$ and $V_{2}$ are different.

When the sample has a high impedance, in order to extract the initial value $V_{20}$, one first has to consider the impedance transformer, which links the sample to the oscilloscope. Our thin film samples have a high resistance. In particular, if the sample resistivity is higher as the input impedance of the transformer, one measures only a fraction of the actual voltage, i.e. we have to use an equivalent circuit as shown in Fig. $(2-12)$ in order to determine the true voltage $V_{20}$. 


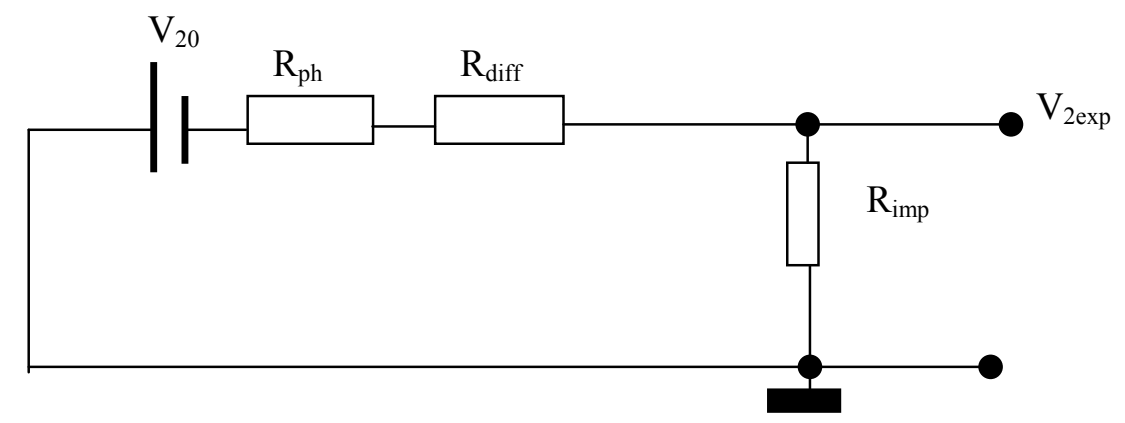

Fig.(2-12) The schematic diagram of the equivalent circuit for the TTE voltage measurements.

In addition the sample decomposes into a dark and illuminated part $R_{d}$ and $R_{p h}$. Thus, in order to determine the signal voltage reduction, one has to measure also the dark conductivity, $R_{d} \sim \rho_{d}$ (Fig.2-12 and 2-14). As the temperature settings for the TTEmeasurement and the resistivity measurement are different, usually one has to use a data interpolation procedure. For that, we have used the smooth function $\ln \rho / \rho_{o}=\left(T / T_{o}\right)^{1 / n}$, which is supposed to interpolate between the crystalline case ( $\mathrm{n}=1$; Arrhenius law) and the amorphous case $(n=4$, Mott law)[91]. However, the use of the dark conductivity for the whole sample is not entirely correct as the sample actually has an illuminated part that can have a much lower (photo-) resistivity, $\mathrm{R}_{\mathrm{ph}}$, and as the dark part can be flooded by charge carriers and as such, also can have a higher conductivity Rdiff. If the equivalent circuit thus is extended to contain a series of $R_{p h}+R_{\text {diff }}$, then:

$$
\mathrm{V}_{20}=\mathrm{V}_{2 \exp }\left(\mathrm{R}_{\mathrm{ph}}+\mathrm{R}_{\text {diff }}+\mathrm{R}_{\mathrm{imp}}\right) / \mathrm{R}_{\mathrm{imp}}
$$

Assuming that $\mathrm{R}_{\text {diff }}$ still has the same temperature dependence as $\mathrm{R}_{\text {dark }}$, but a different prefactor and that $\mathrm{R}_{\mathrm{ph}}$ is approximately constant with the temperature, the correction involves only two parameters :

$$
\mathrm{V}_{20}=\mathrm{V}_{2 \exp }\left(\alpha \rho_{\mathrm{d}}(\mathrm{T})+\beta\right)
$$

where $\alpha$ can in principle be determined by measuring the photoconductivity while $\beta \approx 1$, at least at high temperatures.

As $V_{20}$ and $\tau_{2}$ are interrelated, one can now test the correction: one should recover the measured time constant using

$$
1 / \tau_{2}=(1 / 2 \tau)\left(1+y^{2}-y\left(2+y^{2}\right)^{1 / 2}\right)
$$


where $\mathrm{y}=\alpha \mathrm{eV}_{20} / 2 \mathrm{kT}$ and $\tau$ is an average relaxation time. In the simplest approximation $\tau_{1} \leq \tau<\tau_{2}$. As $\tau_{1}$ is usually in the order of microseconds, this condition is usually fulfilled. Fig.(2-13) shows that $\tau_{2 \exp }=\tau_{2}$ as calculated from $V_{20}$ is true only at high temperatures.

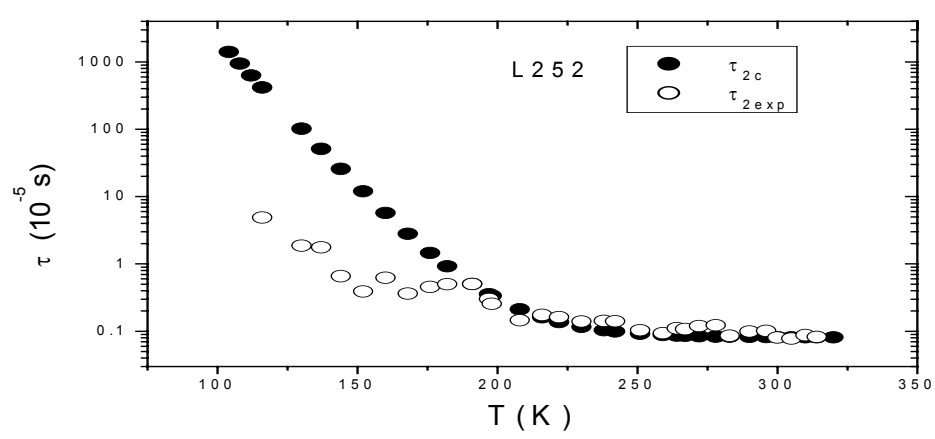

Fig.(2-13) The experimental and calculated relaxation time $\tau_{2}$ for the poly-Si:H film sample L252.

However, in tendency, we are able to confirm the correlation of amplitude $\mathrm{V}_{20}$ and time constant $\tau_{2}$ as predicted by theory. From that we may conclude the existence of space charges and with it the existence of trap states. However, the difference at low temperatures suggests that we should differentiate between a local voltage $\mathrm{V}^{\mathrm{L}}{ }_{20}$ as determined from $\tau_{2}$ and a directly measured global voltage $\mathrm{V}^{\mathrm{g}}{ }_{20}$.

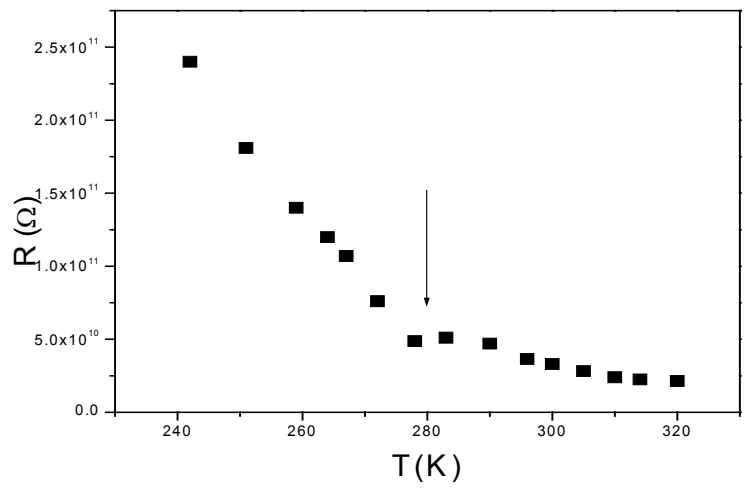

Fig.(2-14) The resistance for the sample L252 (poly-Si:H) versus the temperature.

The use of the global $\mathrm{V}^{\mathrm{g}}{ }_{20}$ might not be the best method for determining the trap density of states as thermovoltages from stage 3 , and errors in the geometry between illuminated and dark regions might be involved. Therefore, we better use the relaxation 
time $\tau_{2}$ to calculate the local voltage $\mathrm{V}^{\mathrm{L}}{ }_{20}$. According to Eq.(2-34), the relation between the relaxation time $\tau_{2}$ and the TTE-voltage, $V_{20}$ is given by:

$2 / \tau_{2} \approx 1 / \tau-\left(\mathrm{eV}_{02} / \mathrm{kT}\right) / \sqrt{\tau_{1} \tau_{2}}$

if $\tau_{1}=\tau$, we can rewrite to get the $\mathrm{V}^{\mathrm{L}}{ }_{20}$ as a function of the relaxation time $\tau_{2}$,

$$
\mathrm{V}_{20}=\frac{\mathrm{KT}}{\mathrm{e}}\left(\sqrt{\frac{\tau_{2}}{\tau}}\right)\left[1-\frac{2 \tau}{\tau_{2}}\right]
$$

Fig.(2-15) shows the so calculated TTE-voltage as a function of the temperature for sample L252. The voltage calculated from $\tau_{2}$ shows more structure than that taken from the transient amplitude directly. This means that we can get more detailed band tail structure information from $\mathrm{V}^{\mathrm{L}}{ }_{20}\left(\tau_{2}\right)$.

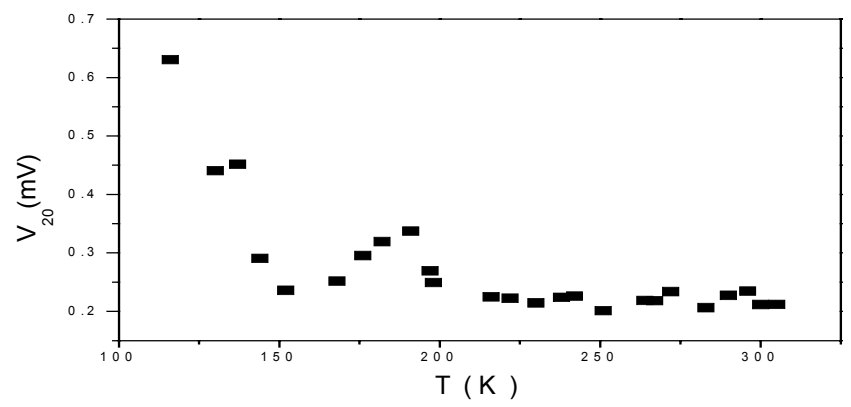

Fig. (2-15) the calculated $\mathrm{V}_{20}$ of poly-Si:H sample L252 versus the temperature.

\subsection{Photoluminescence and small angle $X$-ray scattering (SAXS)}

\subsubsection{Photoluminescence}

Photoluminescence spectroscopy is a contactless, nondestructive method of probing the electronic structure of materials. Light is directed onto a sample, where it is absorbed and imparts excess energy into the material in a process called "photoexcitation." One way this excess energy can be dissipated by the sample is through the emission of light, or luminescence. In the case of photo-excitation, this luminescence is called "photoluminescence." The intensity and spectral content of this photoluminescence can be used to gain insight into various important material parameters such as material composition, defects, etc. 
Specifically, photo-excitation causes electrons within the material to move into permissible excited states. When these electrons return to their equilibrium states, the excess energy is released and may include the emission of light (a radiative process) or may not (a nonradiative process). The energy of the emitted light-or photoluminescenceis related to the difference in energy between the two electron states involved in the transition-that is, between the excited state and the equilibrium state. The quantity of the emitted light is proportional to the relative contribution of the radiative process. Photoluminescence in semiconductors is usually done using a laser.

Here, an Argon laser beam was focused on the sample, which is located in the center of the sample compartment, as seen in Fig.(2-16). If the energy of photons coming from the laser source is greater than the energy gap of the semiconductor, the sample emits photons. These are collected and analyzed with a dual flat field spectrograph. Two detectors are used, a CCD and an InGaAs array. This system allows investigations from 0.75 to $2.4 \mathrm{eV}$.

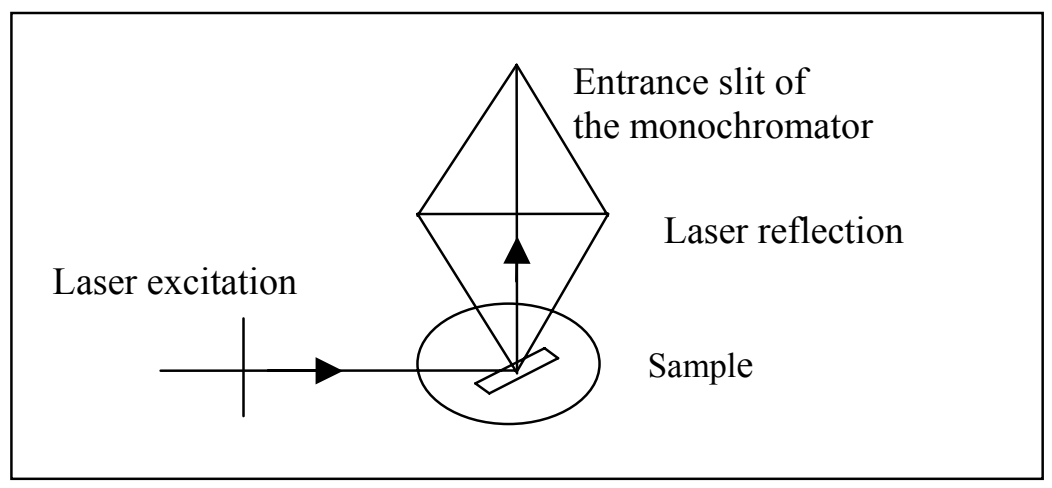

Fig.(2-16) The measurement principle of the photoluminescence.

\subsubsection{Small angle $X$-ray scattering (SAXS)}

SAXS is a standard probe to check on sample quality, too. The usual configuration in the small angle X-ray scattering SAXS experiments is the transmission geometry such that the momentum transfer vector $\mathbf{q}$ lies in the plane of the sample film. This allows study of material density fluctuations parallel to $\mathbf{q}$ in the order of $1 / \mathbf{q}$. The SAXS intensity is collected over a range of $\mathbf{q}$ and plotted versus the magnitude $q=$ $(4 \pi / \lambda) \sin \theta$, where $2 \theta$ is the scattering angle and $\lambda$ is the X-ray wavelength. Anisotropic scattering objects give rise to non-spherical scattering. That preferred orientation in the 
sample can be revealed with two-dimensional detectors or by tilting the sample in the beam so that $\mathbf{q}$ no longer lies in the plane of the sample. There are numerous methods to invert the SAXS intensity $\mathrm{I}(\mathbf{q})$ from momentum space to real space in order to extract structural details of the scattering objects such as their shape, size distribution, preferred orientation, and spatial correlation[92].

To interpret the intensity from small-angle scattering experiments it is convenient to divide it into three components:

$$
\mathrm{I}(\mathbf{q})=\mathrm{I}_{\mathrm{L}}(\mathbf{q})+\mathrm{I}_{\mathrm{N}}(\mathbf{q})+\mathrm{I}_{\mathrm{D}}
$$

where we now assume that there is no preferred orientation of the scattering objects so that only the magnitude of $q$ is relevant. $I_{L}(\mathbf{q})$ represents the intensity from large-scale objects, i.e. objects which are that large that their primary scattering occurs well below the lowest angle accessible by the experimental apparatus. Thus only the tail of the scattering curve contains large objects and it usually exhibits a power-law dependence (Porod law):

$$
\mathrm{I}_{\mathrm{L}}(\mathbf{q})=\mathrm{Aq}^{\mathrm{s}}
$$

where $\mathrm{A}$ is a constant related to the total surface area of the objects and $\mathrm{s}=-4$ for pointfocus SAXS systems while $\mathrm{s}=-3$ for line focus systems [92].

$\mathrm{I}_{\mathrm{N}}(\mathbf{q})$ is of primary interest as it represents the intensity due to nanostructural features. The size range readily accessible by SAXS is typically from about $1 \mathrm{~nm}$ to 100 $\mathrm{nm}$. If the scattering object is a well-defined particle of volume (v) and uniform electron scattering length density $n_{p}$ imbedded in a matrix of density $n_{m}$, then $I_{N}(q)$ for a single particle can be expressed in terms of a form factor $\mathrm{P}(\mathbf{q})$ determined by the shape of the particle:

$$
\mathrm{I}_{\mathrm{N}}(\mathbf{q})=\mathrm{Cv}^{2}\left(\mathrm{n}_{\mathrm{p}}-\mathrm{n}_{\mathrm{m}}\right)^{2} \mathrm{P}(\mathbf{q})^{2}
$$

$\mathrm{C}$ is a constant depending on the choice of radiation and units. A wide variety of particle shapes and their associated form factors have been considered in the interpretation of small-angle data [92]. The total intensity from distributions of particles of small shape is obtained by superposition via Eq.(2-60). If the particle density is high or the particles are correlated in position due to some type of interactions, then interparticle interference will influence $\mathrm{I}_{\mathrm{N}}(\mathbf{q})$ and a pair correlation structure factor $\mathrm{S}(\mathbf{q})$ should be included [92]. 
Experimental evidence for this effect is a maximum or shoulder in $\mathrm{I}(\mathbf{q})$ that is not expected from $\mathrm{P}(\mathbf{q})$ alone.

There is special technique to vary the intensity scattering contrast in SAXS experiments and extract more information on the nanostructure term $\mathrm{I}_{\mathrm{N}}(\mathbf{q})$ : the method of anomalous SAXS. ASAXS allows the tuning of the contrast by varying the wavelength close to the absorption edge of a particular element and thereby highlighting any nanostructure associated with that particular element [93]. This method has recently been applied to a-SiGe:H alloys to search for Ge composition fluctuations[94].

The third term contributing the SAXS intensity (Eq.(2-58)) is that due to diffuse scattering, $\mathrm{I}_{\mathrm{D}}$. It has a variety of sources, all of which are at the atomic scale so that there is little or no $\mathbf{q}$ dependence over typical experimental ranges. Although $\mathrm{I}_{\mathrm{D}}$ is weak for $\mathrm{X}$ ray scattering, it is readily detectable in our experiments by either a nearly $\mathbf{q}$-independent intensity over a wide range of $\mathbf{q}$ or the approach to a constant intensity level at high $\mathbf{q}$. Sources for this intensity include thermal diffuse scattering, static disorder scattering, Compton incoherent scattering and Laue monotonic scattering [95]. Theoretical expressions for the latter effect suggest that information on alloy randomness may be extracted [95]. The experiments were carried out as follows:

Films $2-4 \mu \mathrm{m}$ thick were deposited onto $10 \mu \mathrm{mX} 2.5 \mathrm{cmX} 2.5 \mathrm{~cm}$ iron-free Al foil for SAXS measurements. The SAXS measurements was done with a Kratky compact small angle system attached to a Rigaku rotating anode $(\mathrm{Cu}) \mathrm{X}$-ray generator and the $\mathrm{Cu} \mathrm{K}_{\alpha}$ radiation was selected with a crystal monochromator. The line-collimated X-ray beam was $150 \mu \mathrm{m}$ X $2.2 \mathrm{~cm}$ at the sample. The sizes of entrance slit $(150 \mu \mathrm{m})$ and detector slit $(375 \mu \mathrm{m})$ of the SAXS system were chosen to yield a suitable resolution while maintaining an adequate scattering signal. The above settings allow detection of particles or density fluctuations with a maximum size of about $26 \mathrm{~nm}$. The scattered X-rays were detected with an Ar-Xe gas field proportional counter that has a peak efficiency of $90 \%$ very near to $8.05 \mathrm{KeV} \mathrm{Cu} \mathrm{K} \mathrm{K}_{\alpha}$ energy. The background (or dark) count rate $\mathrm{I}_{\mathrm{d}}$, obtained with no radiation allowed into the SAXS system from X-ray generator, is a nearly constant value of 0.20 count/s using a single channel analyzer window centered on the $\mathrm{Cu}$ $\mathrm{K}_{\alpha} \mathrm{X}$-ray. The raw data consisted of the measured X-ray count rate $\mathrm{I}_{\mathrm{m}}$ versus the magnitude of the scattering vector, $\mathrm{q}=2 \pi(2 \theta) / \lambda$, for a scan of the scattering angle $2 \theta$ 
from $0.16^{\circ}$ to $8.4^{\circ}$. This was done for both the sample plus substrate, $I_{m}(q)$, and for the substrate alone, $I_{s}(q)$. To obtain the SAXS intensity from the film alone, corrections were then made for $I_{d}$, for scattering and absorption by Al foil substrate, and for absorption by the film itself; the intensity was then normalized by the absolute intensity of the direct beam $\mathrm{P}_{\mathrm{o}}$, and by the product of film thickness, $\mathrm{t}_{\mathrm{f}}$, and absorption coefficient, $\mu_{\mathrm{f}}$ [96] using the equation:

$$
I(q)=\frac{\left[I_{m}(q)-I_{d}\right]-\left[I_{s}(q)-I_{d}\right] \exp \left(-\mu_{f} t_{f}\right)}{P_{o} \mu_{f} t_{f} \exp \left(-\mu_{f} t_{f}-\mu_{s} t_{s}\right)}
$$

$\mathrm{P}_{\mathrm{o}}$ was obtained by the moving slit technique [97] and was typically about $10^{8}$ count/s, while the value of $\mu_{\mathrm{f}} \mathrm{t}_{\mathrm{f}}$ was determined by measuring the ratio of the intensities of the direct beam through the film plus substrate, $P_{o} \exp \left(-\mu_{\mathrm{f}} \mathrm{t}_{\mathrm{f}}-\mu_{\mathrm{s}} \mathrm{t}_{\mathrm{s}}\right)$, and through the substrate only, $\mathrm{P}_{\mathrm{o}} \exp \left(-\mu_{\mathrm{s}} \mathrm{t}_{\mathrm{s}}\right)$. One measurement was made with the sample normal tilted at an angle of $45^{\circ}$ to the beam, in which case $I_{s}(q)$ was also measured with the substrate tilted at the same angle.

\subsection{Samples preparation:}

\subsubsection{The hot wire chemical vapor deposition of poly-Si:H high deposition rate samples}

Poly-Si:H films were grown by B.P. Nelson at National Renewable Energy Laboratory (NREL), in a tube-reactor (tube-heater), as illustrated in Fig.(2-17), He used the HWCVD technique and deposition rates ranging from 50 to $130 \AA \AA$ s. The high deposition rates were achieved by adding a second filament, decreasing the filament-tosubstrate distance $(5 \mathrm{~cm}-3.2 \mathrm{~cm})$, increasing deposition pressure $(10-20$ mTorr - 30-70 mTorr), and the silane flow rate $(20-50 \mathrm{sccm}-40-75 \mathrm{sccm})$. The substrate temperature was in the range of $200-400^{\circ} \mathrm{C}$, and the filament current was fixed to $30 \mathrm{~A}$. Table (2-1) shows the specification of the preparation conditions and some properties of the high deposition rate poly-Si:H samples. 
Table (2-1) Growth condition and properties of high deposition rate HWCVD poly-Si:H samples. Where $\mathrm{T}$ is the substrate temperature, $\mathrm{P}$ is the partial pressure of the gas within the deposition chamber, $\mathrm{F}$ is the silane flow rate, $\mathrm{R}$ is the deposition rate and $\mathrm{E}_{04}$ is the Urbach energy.

\begin{tabular}{|c|c|c|c|c|c|c|c|}
\hline Sample & $\mathrm{T}$ & $\mathrm{P}$ & $\mathrm{F}\left(\mathrm{SiH}_{4}\right)$ & Thickness & $\mathrm{R}$ & $\begin{array}{c}\text { Photo } \\
\text { Conductivity } \\
\mathrm{S} / \mathrm{cm}\end{array}$ & $\mathrm{E}_{04}$ \\
\hline $\mathrm{ID}$ & ${ }^{\circ} \mathrm{C}$ & $\mathrm{mTorr}$ & $\mathrm{sccm}$ & $\AA$ & $\AA$ & $\mathrm{A} / \mathrm{s}$ & \\
\hline L256 & 316 & 30 & 40 & 19715 & 50 & $2.36 \times 10^{-4}$ & 1.83 \\
\hline L253 & 316 & 35 & 50 & 19266 & 70 & $2.64 \times 10^{-4}$ & 1.83 \\
\hline L254 & 316 & 50 & 50 & 19084 & 90 & $3.71 \times 10^{-4}$ & 1.84 \\
\hline L255 & 325 & 70 & 75 & 20375 & 130 & $1.61 \times 10^{-4}$ & 1.82 \\
\hline
\end{tabular}

Side View

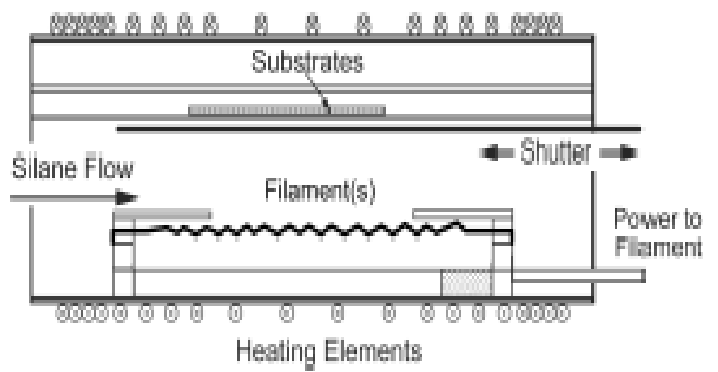

End Views

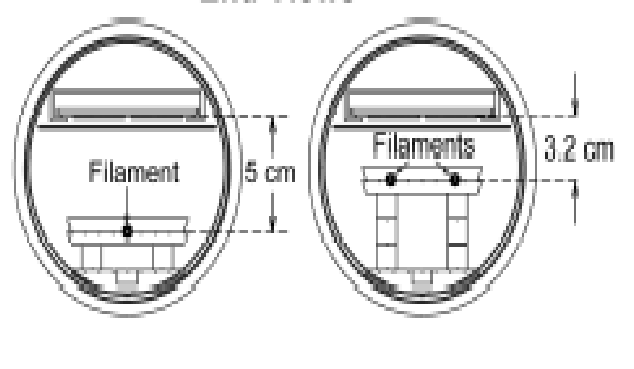

Fig.(2-17) A cross-section of the tube-heater in which poly-Si:H films were grown by HWCVD. Note how the filament clamping arrangement allows for changing the number of filaments and the filament-to-substrate distance.

\subsubsection{The hot wire chemical vapor deposition (HWCVD) of a-SiGe:H alloys:}

B.P. Nelson at NREL, also grew these samples again using hot wire chemical vapor deposition (HWCVD). Specifically, these samples were grown on glass substrate (1737Corning), using a $0.38 \mathrm{~mm} \mathrm{~W}$ (tungsten wire) filament wrapped in coil in a tube reactor using an isothermal heating zone. The distance between the filament and the substrate was $5.0 \mathrm{~cm}$. Germane and silane were the Ge and Si source gases, respectively. 
Hydrogen dilution was used at flows similar to the sum of the flows of the silane and germane. Additional preparation details are in Table (2-2) and reference [98].

Table (2-2) the preparation parameters of HWCVD a-SiGe:H samples. Where $\mathrm{T}_{\mathrm{S}, \mathrm{E}}$ is the starting and ending substrate temperature, $\mathrm{R}$ is the deposition rate, $\mathrm{D}_{\mathrm{P}}$ is the deposition pressure, $\mathrm{E}_{04}$ is the Urbach energy, the Tauc gap is the optical energy gap, $\mathrm{n}$ is the refractive index and $\mathrm{k}$ is the extinction coefficient.

\begin{tabular}{|c|c|c|c|c|c|c|c|c|c|c|}
\hline $\begin{array}{l}\text { Sample } \\
\text { ID }\end{array}$ & $\begin{array}{l}\mathrm{SiH}_{4} \\
\text { Flow } \\
\text { rate } \\
\text { sccm }\end{array}$ & $\begin{array}{l}\mathrm{GeH}_{4} \\
\text { Flow } \\
\text { rate } \\
\text { sccm }\end{array}$ & $\begin{array}{l}\mathrm{H}_{2} \\
\text { Flow } \\
\text { rate } \\
\text { sccm }\end{array}$ & $\mathrm{m}$ Torr & $\mathrm{T}_{\mathrm{S}}$ & $\mathrm{T}_{\mathrm{E}}$ & $\begin{array}{l}\text { Film } \\
\text { Thickness } \\
\AA\end{array}$ & $\AA / S$ & $\begin{array}{l}\mathrm{E}_{04} \\
\text { by } \\
\mathrm{n} \& \mathrm{k} \\
\mathrm{eV}\end{array}$ & $\begin{array}{l}\text { Tauc gap } \\
\text { from } \\
\mathrm{n \& k} \\
\mathrm{eV}\end{array}$ \\
\hline L730 & 16.25 & 8.75 & 25 & 15 & 250 & 315 & 9647 & 8.04 & 1.47 & 1.2931 \\
\hline L732 & 16.25 & 8.75 & 25 & 15 & 250 & 315 & 8779 & 7.32 & 1.47 & 1.2965 \\
\hline L733 & 18.75 & 6.25 & 25 & 15 & 250 & 316 & 9298 & 6.74 & 1.58 & 1.3921 \\
\hline L740 & 16.25 & 8.75 & 25 & 15 & 250 & 290 & 2055 & 3.11 & 1.45 & 1.3281 \\
\hline L743 & 18.75 & 6.25 & 25 & 15 & 250 & 309 & 3221 & 4.13 & 1.51 & 1.3745 \\
\hline L744 & 16.25 & 8.75 & 25 & 15 & 250 & 303 & 3485 & 4.84 & 1.43 & 1.294 \\
\hline L745 & 16.25 & 8.75 & 25 & 15 & 125 & 200 & 1380 & 0.58 & 1.14 & 1.1657 \\
\hline L749 & 16.25 & 8.75 & 25 & 15 & 250 & 300 & 5378 & 8.96 & 1.5 & 1.3438 \\
\hline L751 & 16.25 & 8.75 & 25 & 15 & 125 & 187 & 1761 & 1.96 & 1.5 & 1.3901 \\
\hline
\end{tabular}

\subsubsection{The low pressure reactive ECR plasma $\mu \mathrm{c}-\mathrm{SiGe:H}$ samples}

The samples were grown using a remote ECR plasma process that is described in reference [99]. The feedstock gases were silane $\left(\mathrm{SiH}_{4}\right)$ and germane $\left(\mathrm{GeH}_{4}\right)$, or germane alone. A significant hydrogen dilution ratio ( $>20: 1)$ was used for all samples. The typical deposition temperatures were in the range of $250-300^{\circ} \mathrm{C}$, and the microwave power levels were $150 \mathrm{~W}$. The pressure during deposition was maintained lower than $10 \mathrm{mTorr}$ so as to make sure that a significant ion flux was impinging on the substrate during growth since we had experienced previously that low pressure deposition led to the best films and devices [100]. The growth rates were $\sim 1 \AA / \mathrm{s}$. A subtle (sub ppm) B doping was added to the samples to passivate any native donor-like impurities, such as $\mathrm{O}$, N, etc., compensate 
dangling bonds and release the local strain in the network [101,102]. Table (2-3) shows the preparation parameters of the low pressure reactive ECR plasma $\mu \mathrm{c}-\mathrm{SiGe}: \mathrm{H}$ samples.

Table (2-3) Growth conditions of the low pressure reactive ECR plasma $\mu \mathrm{c}-\mathrm{SiGe}: \mathrm{H}$ samples. Where $\mathrm{T}$ is the substrate temperature, $\mathrm{P}$ is the partial pressure of the gas within the deposition chamber, time is the deposition time and SCCM is the Standardized Cubic Centimeters per Minute.

\begin{tabular}{|c|l|l|l|l|l|l|l|}
\hline $\begin{array}{c}\text { Sample } \\
\text { ID }\end{array}$ & $\begin{array}{c}\text { Time } \\
(\mathrm{s})\end{array}$ & $\begin{array}{c}\mathrm{T} \\
\left({ }^{\circ} \mathrm{C}\right)\end{array}$ & $\begin{array}{c}\mathrm{P} \\
(\mathrm{mT} \text { Torr })\end{array}$ & $\begin{array}{c}\mathrm{H}_{2} \\
\mathrm{SCCM}\end{array}$ & $\begin{array}{c}\mathrm{SiH}_{4} \\
\mathrm{SCCM}\end{array}$ & $\begin{array}{c}\mathrm{GeH}_{4} \\
\mathrm{SCCM}\end{array}$ & $\begin{array}{c}\text { Thickness } \\
(\mu \mathrm{m})\end{array}$ \\
\hline $2 / 5986$ & 120 & 300 & 5 & 72 & 0.65 & 0.18 & 0.56 \\
\hline $2 / 5987$ & 120 & 300 & 5 & 72 & 0.65 & 0.18 & 0.46 \\
\hline $2 / 5995$ & 120 & 250 & 5 & 30 & 1.3 & 0.18 & 0.99 \\
\hline $2 / 5996$ & 120 & 250 & 6 & 72 & 1.3 & 0.18 & 0.71 \\
\hline $2 / 5997$ & 120 & 250 & 6 & 72 & 0.91 & 0.18 & 0.57 \\
\hline
\end{tabular}




\section{Results}

\subsection{The Photomixing results:}

\subsubsection{Determination of the range and depth of long-range potential fluctuations (LRPF)}

As stated above, the dangling bonds and other defects, which serve both as recombination centers and trapping centers, can be charged and form potential barriers which fluctuate. In the transport process, the charge carriers can either go over the potential barrier through thermal activation or go around the potential barrier through scattering. A model for charge transport in potential barriers due to long- range potential fluctuations was previously developed $[103,70]$. This has enabled us to determine the range of the long-range potential fluctuations in annealed and light-soaked samples of a$\mathrm{Si}: \mathrm{H}$. In this context, we report on the determination of the depth as well as the length of the potentials in our samples. For the purpose of continuity of presentation, we will briefly review the model which is based on the idea that the mobility is proportional to the probability of a carrier going over a potential barrier by thermal activation. The role of the external electric field is (1) to accelerate the charge carriers and (2) to change the density of states inside the potential well; the density of states is assumed to be proportional to the spatial range of the potential well. If the probability of the charge carriers to go over the potential barrier is determined by thermal activation, then through statistical calculations, one can obtain the electric field dependence of the drift mobility $\left(\mu_{d}\right)$ from the following equation:

$$
\mu_{\mathrm{d}}(\mathrm{E})=\mu_{\mathrm{d}}^{0} \exp \left(-\frac{\mathrm{eV} \mathrm{p}}{\mathrm{kT}}\right) \frac{\mathrm{eL} \cdot \mathrm{E}}{\mathrm{kT}\left[1-\exp \left(-\frac{\mathrm{eL} \cdot \mathrm{E}}{\mathrm{kT}}\right)\right]}
$$$$
\text { (if } \left.|\mathrm{L} \cdot \mathrm{E}| \leq V_{P}\right),(3-1)
$$

where $\mathrm{E}$ is the applied electric field, $\mu_{\mathrm{d}}{ }^{\circ}$ the drift mobility without the existence of longrange potential fluctuations, and $\mathrm{V}_{\mathrm{P}}$ and $\mathrm{L}$ are the average depth and the length of the potential fluctuations, respectively. It is seen from Eq. (3-1) that $\mu_{d}(E)$ will increase with decreasing $V_{P}$ and increasing $L$. The applied electric field is a variable. So, by normalizing the values for the drift mobility at the lowest electric field $\mathrm{E}_{1}$ and using Eq. (3-1), we obtain:

$$
\frac{\mu_{d}\left(E_{i}\right)}{\mu_{d}\left(E_{1}\right)}=\frac{E_{i}}{E_{1}} \frac{1-\exp \left(-\frac{e L \cdot E_{1}}{k T}\right)}{1-\exp \left(-\frac{e L \cdot E_{i}}{k T}\right)} \quad(i=1,2,3, \ldots \ldots)
$$


Through curve fitting the normalized mobility data as a function of electric field (Eq. (3-2)), we can obtain the average length $\mathrm{L}$. For a given $\mathrm{E}_{\mathrm{i}}$, the value of normalized mobility will increase with increasing length L, suggesting an enhanced field dependence of the mobility. The drift mobility results obtained from fits can subsequently be used to fit the absolute mobility data to Eq.(3-1) so as to obtain the average depth $\mathrm{V}_{\mathrm{P}}$ of the potential fluctuations in a given sample.

\subsubsection{Estimate of the charged defect density}

The density of charged defects, $n$, is thought to be responsible for the long-range potential fluctuations and can be determined in arbitrary units according to [104]:

$$
\mathrm{n} \propto \mathrm{V}_{\mathrm{P}}^{2} / \mathrm{L}
$$

By using this relationship, we can estimate the charged defect density when we change the preparation parameters of our samples. An increase in the concentration of charged defects will result in a tendency for the depth $V_{P}$ of the potential fluctuations to increase, while an increasing length $\mathrm{L}$ tends to decrease $\mathrm{n}$. In the following sections we present details of the charge transport in our samples:

\subsubsection{Application to the samples}

\subsubsection{The hot wire chemical vapor deposition poly-Si:H high deposition rate samples.}

\section{A: photomixing on the as-deposited samples; state (A)}

The photoconductivity as a function of deposition rate is shown in Fig. (3-1). It is seen that in the deposition range from $40 \AA / \mathrm{s}$ to $\sim 140 \AA / \mathrm{s}$, the photoconductivity hovers at levels of $1 \times 10^{-4} \mathrm{~S} / \mathrm{cm}$; only in the neighborhood of the deposition rate of $90 \AA / \mathrm{s}$, the photoconductivity peaks at about $\sim 2.5 \times 10^{-4} \mathrm{~S} / \mathrm{cm}$.

By measuring the photomixing signal in conjunction with the DC photoconductivity, we determined both the drift mobility and lifetime of the samples. This enables us to investigate in more detail the deposition rate dependence of the sample transport properties with the ultimate aim to find out the origin of the changes in the photoconductivity.

Fig. (3-2) shows the drift mobility as a function of deposition rate. The mobility peaks in the neighborhood of $90 \AA /$ s, i.e. where the photoconductivity peaks. Moreover, the lifetime appears to show a peak in this region too, as can be seen in Fig. (3-3). 


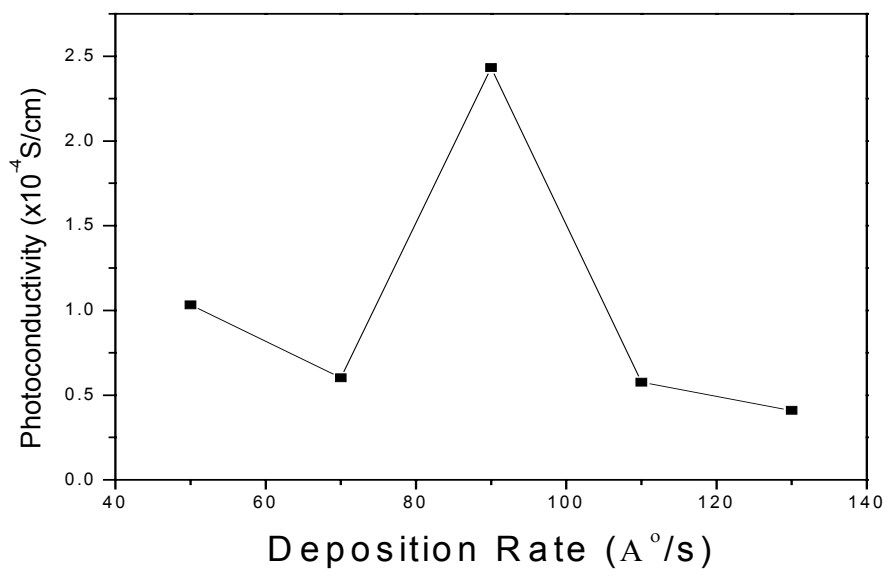

Fig. (3-1) The photoconductivity of poly-Si:H as a function of the deposition rate.

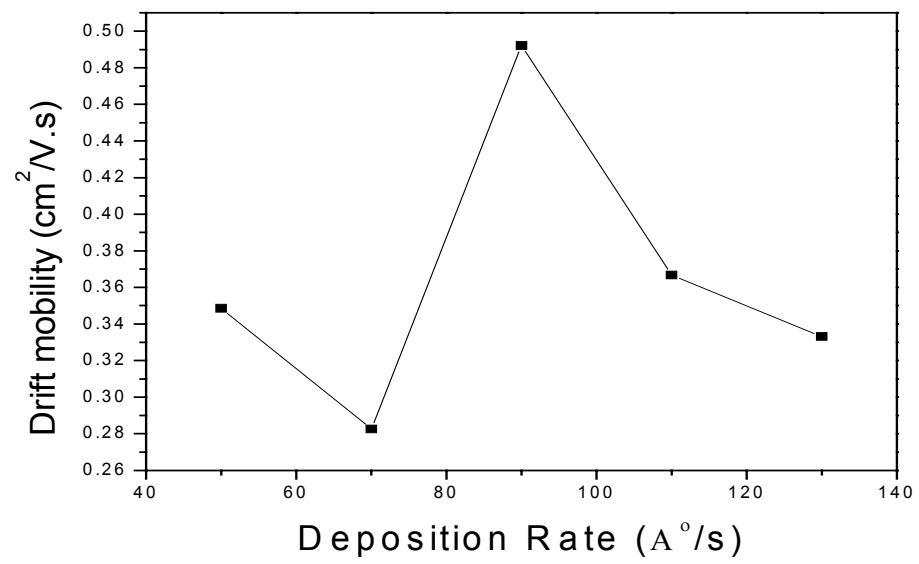

Fig. (3-2) The drift mobility of poly-Si:H as a function of the deposition rate.

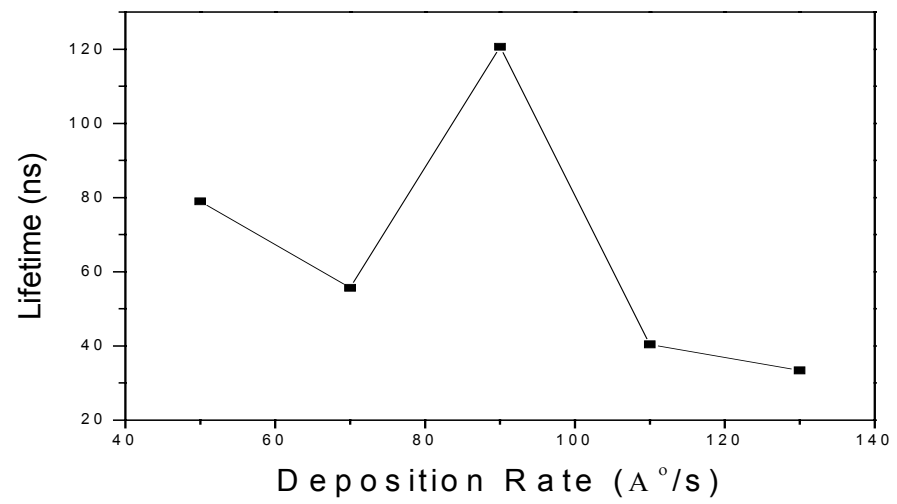

Fig. (3-3) The lifetime of poly-Si:H as a function of the deposition rate. 
The deposition rate dependence of the mobility (Fig. (3-2)) suggests that the longrange potential fluctuations may be involved in the charge transport process. As it was suggested for a-Si:H[103-107], the decrease in the drift mobility results from an increase in the charged scattering centers which in turn are related to the long-range potential fluctuations. Further evidence for potential fluctuations was found in particular from the fact that the mobility increases with increasing applied electric field and with increasing illumination [103]. Specifically, the measurements of the electric field dependence of the mobility and lifetime show strong evidence for the presence of long-range potential fluctuations. Fig. (3-4) shows a typical electric field dependence of the mobility and lifetime. This type of data, we interpret in terms of the length and depth of the long-range potential fluctuations in our samples, and have calculated $\mathrm{L}$ and $\mathrm{V}_{\mathrm{P}}$ accordingly. The results are shown in Fig. (3-5).
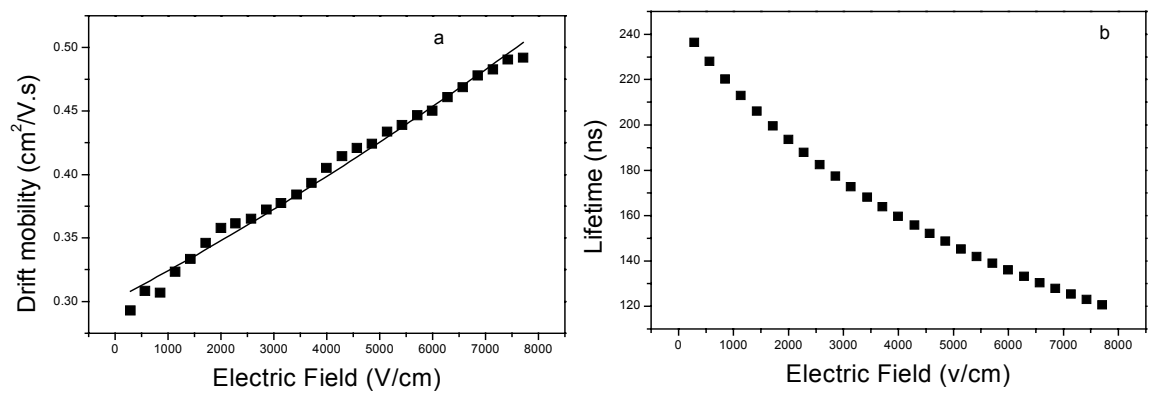

Fig. (3-4) The field dependence of the drift mobility (a) and lifetime (b) for poly-Si:H sample L252.
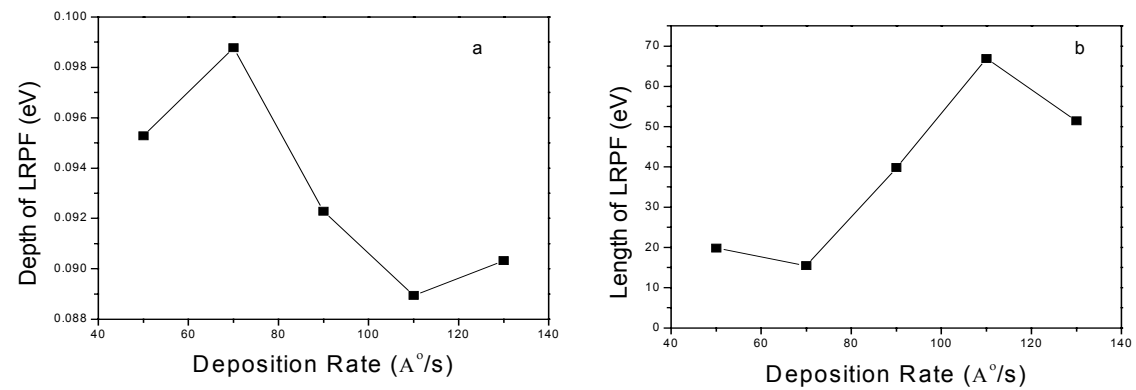

Fig.(3-5) The depth (a) and length (b) of the long-range potential fluctuations as a function of the deposition rate of poly-Si:H samples. 
The density of charged defects (n) that is thought to be responsible for the longrange potential fluctuations can be calculated using Eq.(3-3). Fig. (3-6) shows the relative charge defect density as a function of deposition rate. It is shown that the charge defects density increases with increasing deposition rate up to $\sim 80 \AA / \mathrm{s}$ and then decreases.

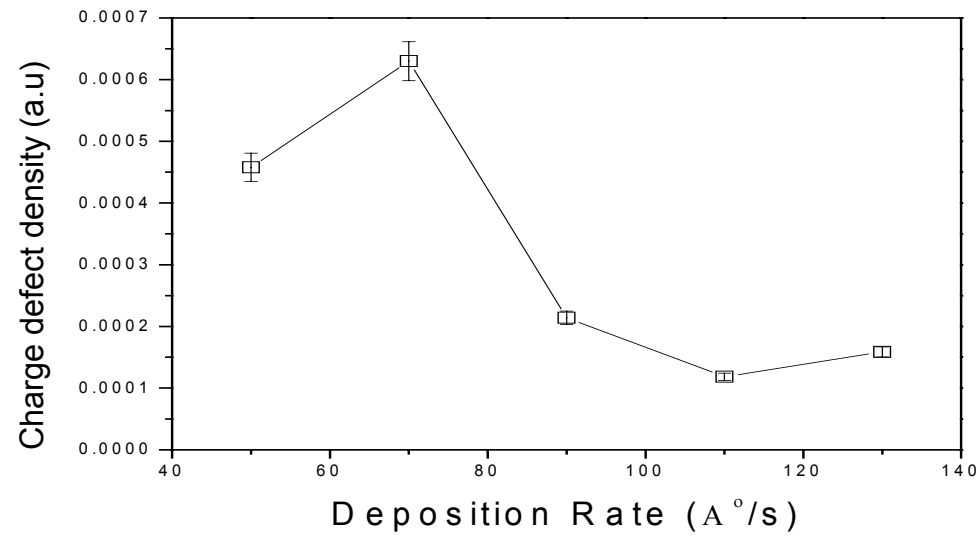

Fig.(3-6) the relative charge defect density versus the deposition rate of poly-Si:H samples.

\section{B. Photomixing of the annealed samples}

Fig. (3-7) shows the photoconductivity, mobility and lifetime of thes samples; in order to obtain the "low temperature annealing" state (B) the samples where kept at 50$70 \mathrm{~K}$ for 24 hours. After annealing the samples for 2 hours at $150{ }^{\circ} \mathrm{C}$, we obtain (state (C)). In both instances, the parameters are given as a function of the deposition rate. Note that the photoconductivity, mobility and lifetime of the high temperature annealed samples (C) have a peak at the neighborhood of the deposition rate $90 \AA / \mathrm{s}$, just like the "as-deposited" samples (A). The "low temperature annealed" samples (B) exhibit different trends and values. Possibly, there is freezing of charged defects and hydrogen reconfiguration still possible at low temperatures. This shows that any low temperature treatment will change the sample properties but that by high temperature annealing one can recover the original sample status.

Again we calculate the depth and length of the long-range potential fluctuations as a function of the deposition rate. Also we estimated the relative charge defect density. 
Fig. (3-8) shows the depth and length of long-range potential fluctuation and the relative charge defect density of state (B) and state (C) samples as a function of the deposition rate.
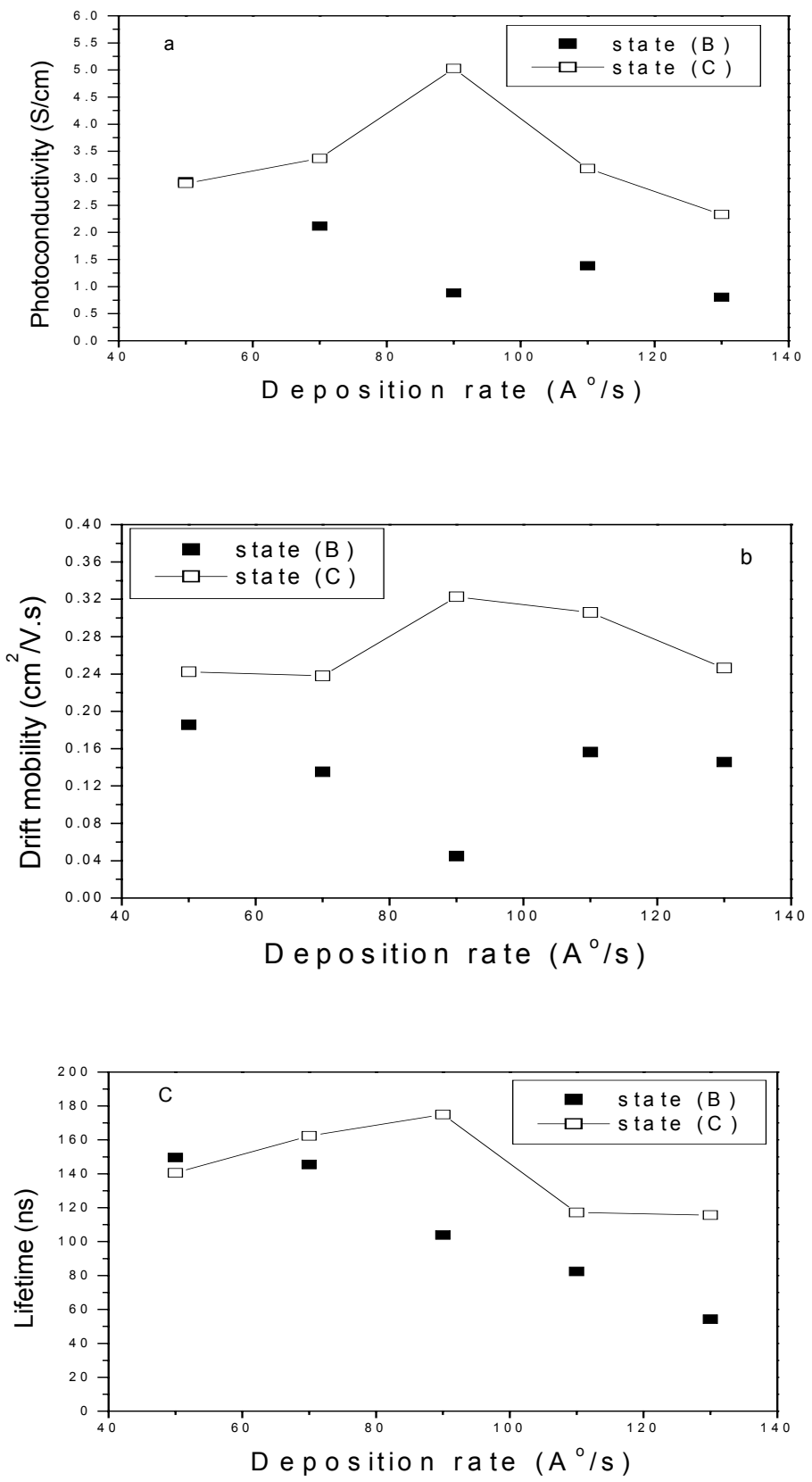

Fig.(3-7) the photoconductivity (a), drift mobility (b) and lifetime (c) versus the deposition rate of state $(\mathrm{B})$ and state $(\mathrm{C})$ samples. 

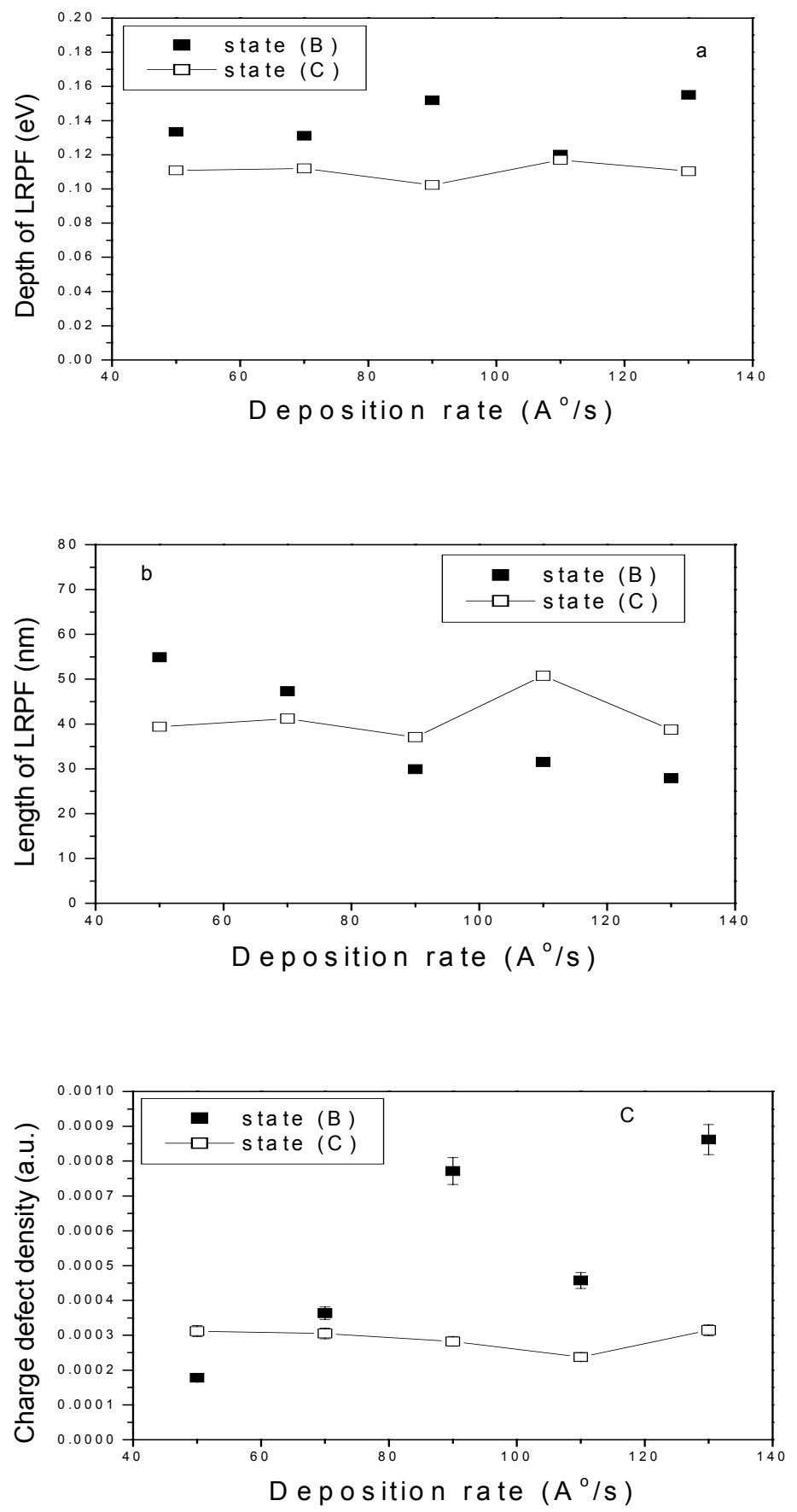

Fig.(3-8) the depth of LRPF (a), length of LRPF (b) and charge defect density (c) versus the deposition rate of state (B) and state (C) samples. 


\subsubsection{The hot wire chemical vapor deposition a-SiGe:H samples.}

Hydrogenated amorphous silicon-germanium alloys (a-SiGe:H) continue to be interest as integral components of multi-layer amorphous silicon-based solar cells. Since, by alloying hydrogenated amorphous silicon (a-Si:H) with germanium, the optical band gap of the material is narrowed, allowing the absorption of the lower energy of the solar spectrum. So, development of low band gap and highly photosensitive a-SiGe:H has become a major task for the fabrication of tandem solar cells for higher efficiency and better stability, however, we will study the correlation between the electronic transport properties and the optical band gap (Tauc gap) of these alloys. The charge transport properties of the hot wire chemical vapor deposition a-SiGe:H samples that were annealed for $2 \mathrm{~h}$ at $150^{\circ} \mathrm{C}$ were characterized by utilizing the photomixing technique. The photoconductivity versus the Tauc gap (the optical band gap) is shown in Fig. (3-9). It is found empirically that with HWCVD the defect density increases with increasing optical (Tauc) gap. It is seen that the photoconductivity indeed decreases with increasing Tauc gap. Fig. (3-10) shows the drift mobility and lifetime as a function of Tauc gap. It is seen that the mobility has the same trend as the photoconductivity; i.e. the mobility decreases with increasing the Tauc gap consistent with a higher defect density; while the lifetime has a more random trend.

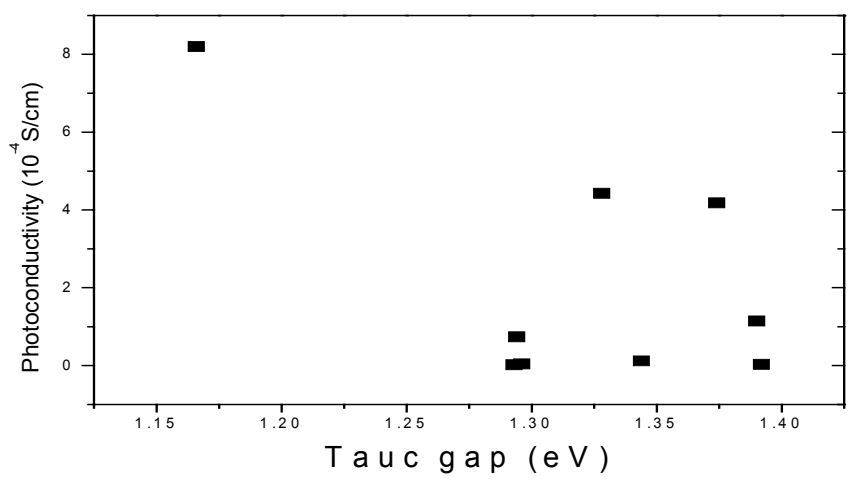

Fig.(3-9) the photoconductivity of HWCVD a-SiGe:H samples versus the Tauc gap. 

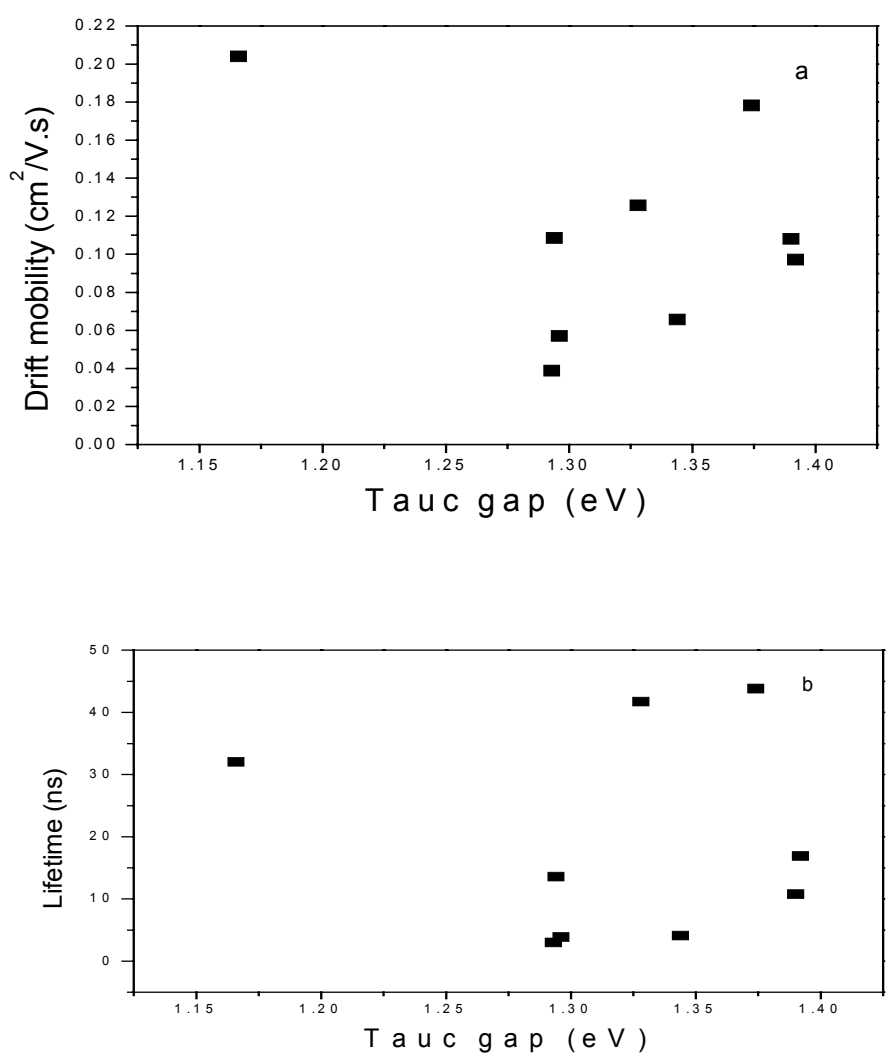

Fig.(3-10) the drift mobility (a) and lifetime (b) of a-SiGe:H samples versus the Tauc gap.

We calculate the depth and length of the long-range of the potential fluctuation and plot them versus the Tauc gap. Also we estimated the relative charge defect density of the samples. Fig. (3-11) shows the depth and length of long-range potential fluctuation and relative charge defect density of the a-SiGe:H samples versus the Tauc gap. The latter indeed increases slightly with the optical gap.

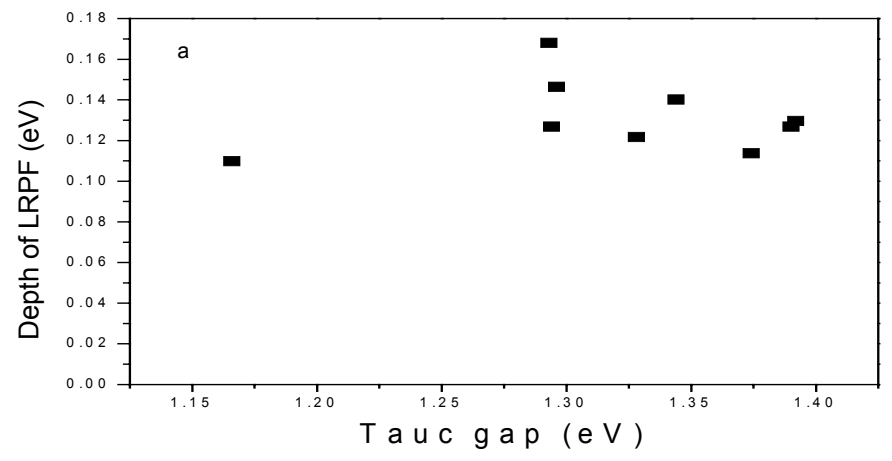



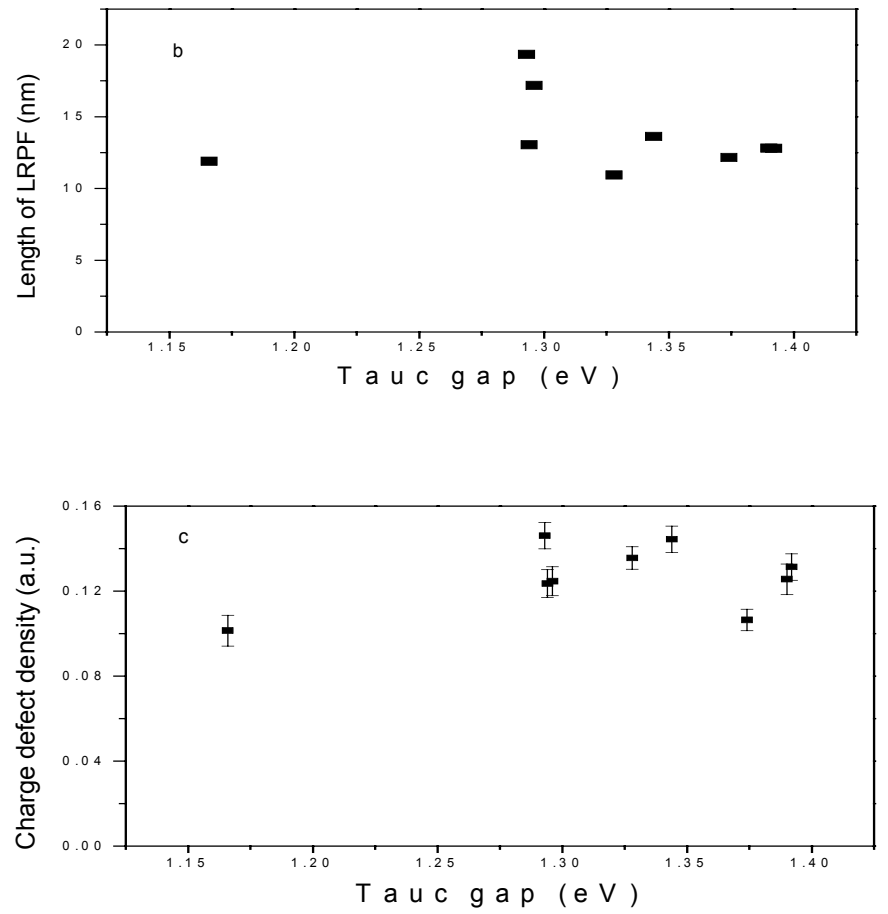

Fig.(3-11) the depth of LRPF (a), length of LRPF (b) and charge defect density (c) versus Tauc gap of HWCVD a-SiGe:H samples.

\subsubsection{The low pressure reactive ECR plasma $\mu \mathrm{c}-\mathrm{SiGe:H}$ samples}

The charge transport properties of the low pressure reactive ECR plasma deposition $\mu \mathrm{c}-\mathrm{SiGe}: \mathrm{H}$ samples that were annealed at $150^{\circ} \mathrm{C}$ for $2 \mathrm{~h}$ were characterized by the photomixing technique. The photoconductivity as a function of the deposition rate is shown in Fig. (3-12). The photoconductivity has peak at $\approx 0.8 \AA / \mathrm{s}$ and decrease when the deposition rate goes a way from this peak value.

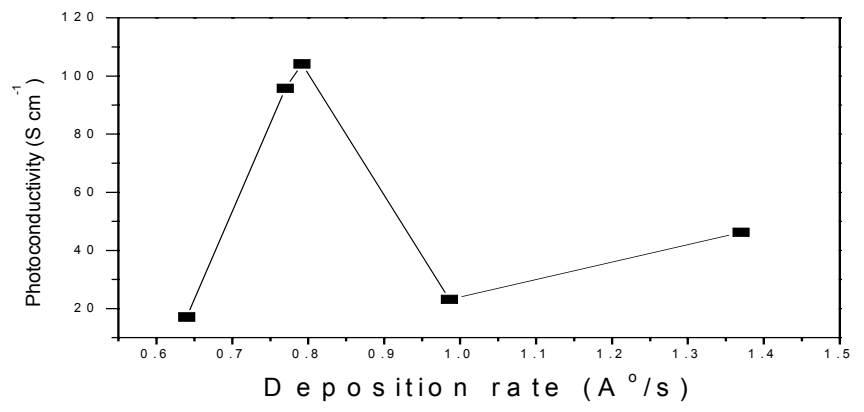

Fig.(3-12) The photoconductivity of the $\mu \mathrm{c}-\mathrm{SiGe}: \mathrm{H}$ samples versus the deposition rate. 
Fig. (3-13) shows the drift mobility and lifetime as a function of the deposition rate of the $\mu \mathrm{c}-\mathrm{SiGe}: \mathrm{H}$ samples. With these samples, the lifetime has the same trend as the photoconductivity; i.e. the lifetime has peak at $\approx 0.8 \AA / \mathrm{s}$, too. In contrast, the mobility has a minimum value at $\approx 0.8 \AA / \mathrm{s}$ and then increases slightly with decreasing the deposition rate.

We also calculated the depth and length of the long-range of the potential fluctuation as a function of the deposition rate. We estimated the relative charge defect density of the samples, too. Fig.(3-14) shows the depth and length of long-range potential fluctuation and relative charge defect density of $\mu \mathrm{c}-\mathrm{SiGe}: \mathrm{H}$ samples as a function of the deposition rate. Again we find that the photomixing results are valuable for materials optimization.
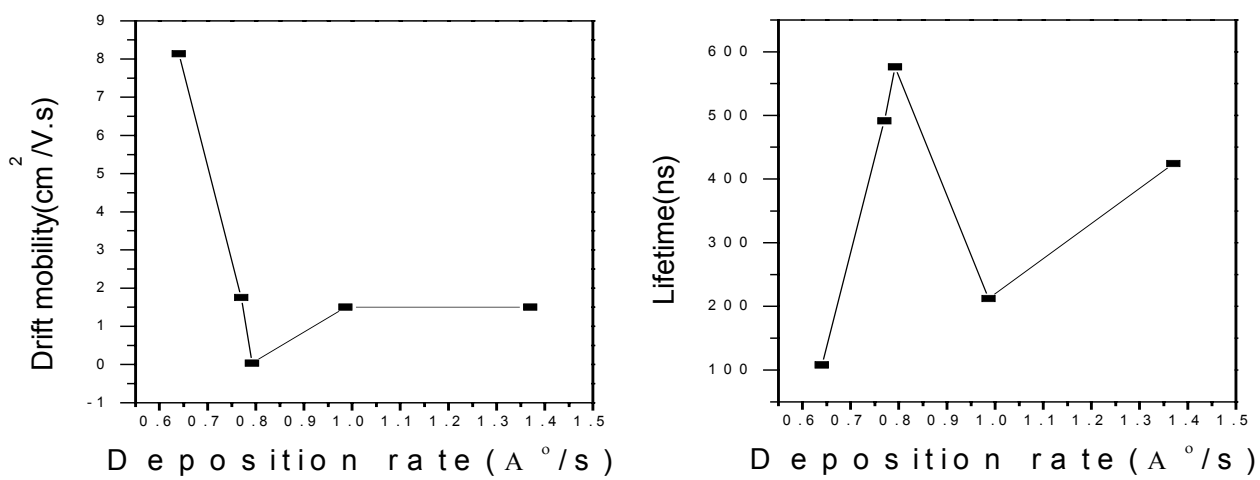

Fig.(3-13) the drift mobility and life time of $\mu \mathrm{c}-\mathrm{SiGe}: \mathrm{H}$ samples versus the deposition rate.

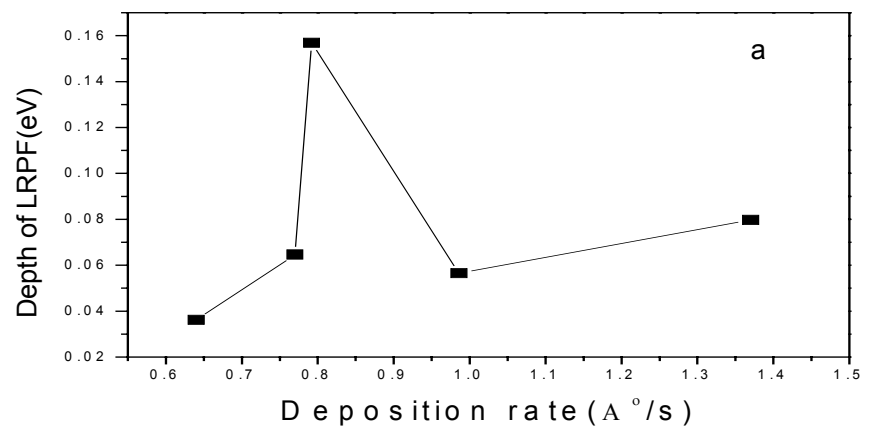



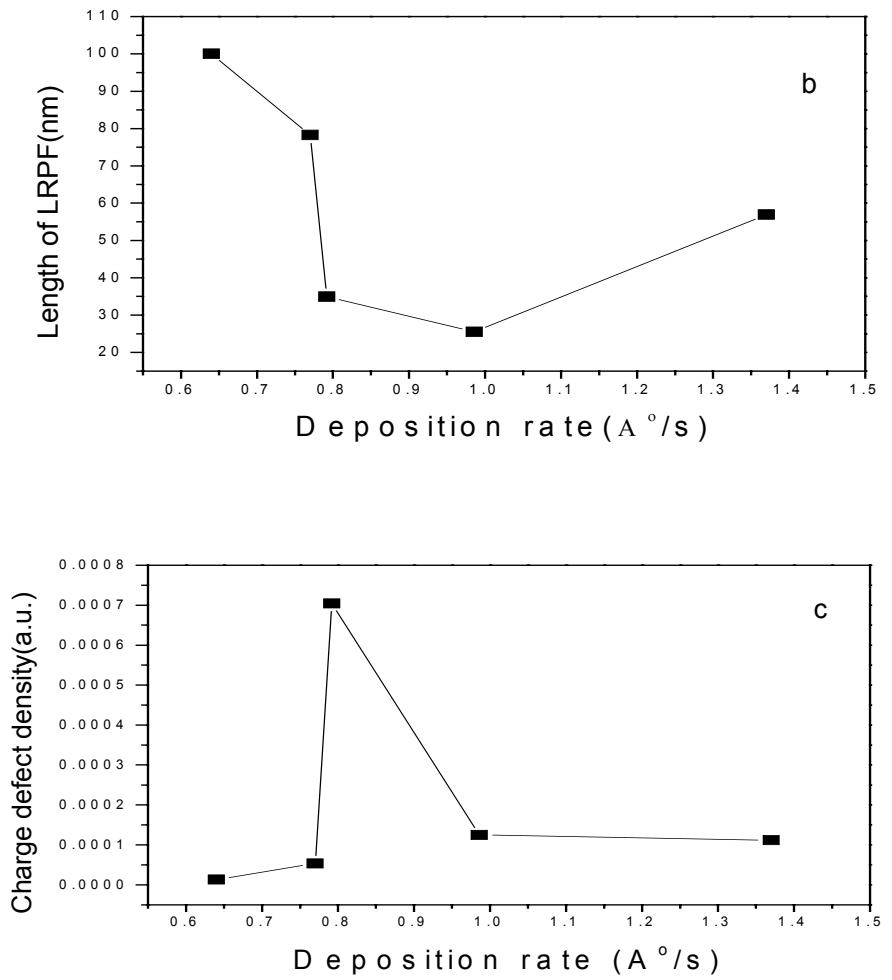

Fig.(3-14) The depth (a) and length (b) of the long-range potential fluctuation and charge defect density (c) of $\mu \mathrm{c}-\mathrm{SiGe}: \mathrm{H}$ samples versus the deposition rate.

\subsection{The TTE Results:}

\subsubsection{The hot wire chemical vapor deposition poly-Si:H high deposition rate samples.}

The poly-Si:H samples were inserted into a TTE-apparatus which was described in detail in Chapter 2. The TTE-voltage signal is measured as a function of time, and the initial voltage $\mathrm{V}_{20}{ }^{*}$ and time constant $\tau_{2}$ are extracted through a fitting routine. Even if the transient voltage shows only weak structure, one can discuss features of the rough $\mathrm{V}^{*} 20$ data already.

Fig (3-15) shows the experimental TTE-voltage signal for poly-Si:H thin film samples as a function of the temperature. It is seen that the rough TTE-voltage $\mathrm{V}_{20}{ }^{*}$ increases with increasing temperature for all samples but differs from sample to sample, thus displaying preparational differences. According to Eq.(2-30) in chapter2 the TTEvoltage can be impedance corrected to yield the global source voltage $V_{20}$. Again, preparational differences show, but in another way. 


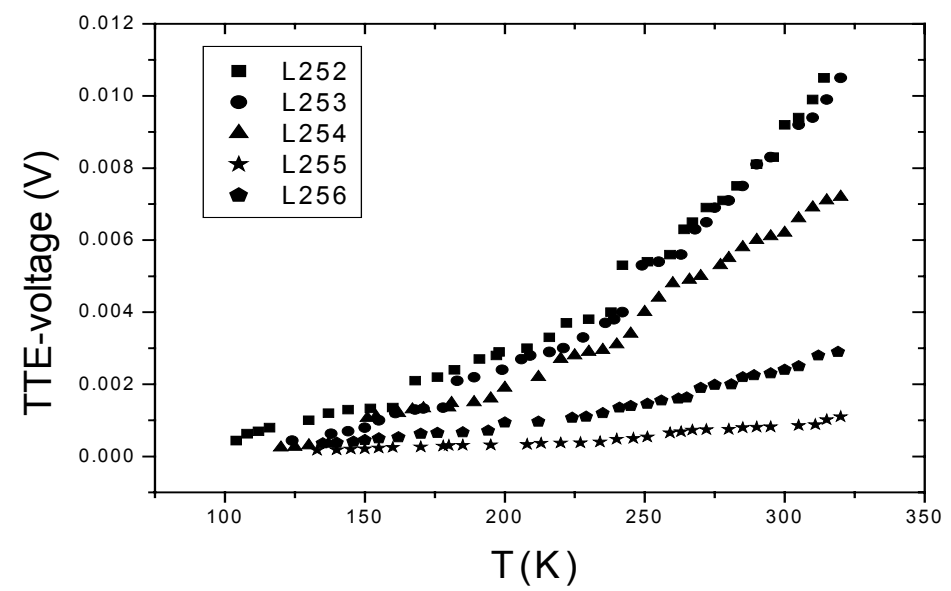

Fig.(3-15) The experimental initial TTE-voltage of poly-Si:H samples versus the temperature.

Fig (3-16) shows the corrected TTE-Voltage of the poly-Si:H samples as a function of temperature. The impedance corrected TTE-voltage $\mathrm{V}_{20}$ increases with decreasing temperature.

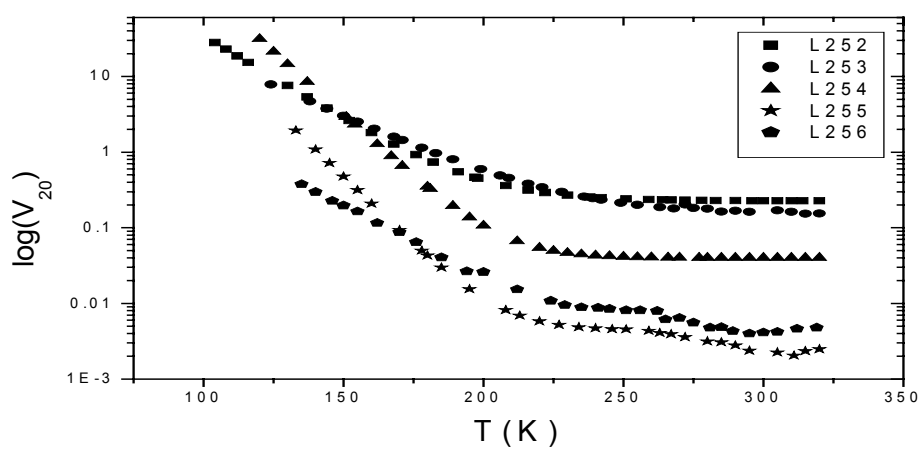

Fig.(3-16) The corrected TTE-voltage of poly-Si:H samples versus the temperature.

Fig.(3-17) shows the corrected voltage $\mathrm{V}_{20}$ of the poly-Si:H films as a function of the deposition rate. $\mathrm{V}_{20}$ shows a peak around the deposition rate $\approx 90 \AA / \mathrm{s}$ and decreases when the deposition rate goes away from the peak value, similar to the photomixing results. 


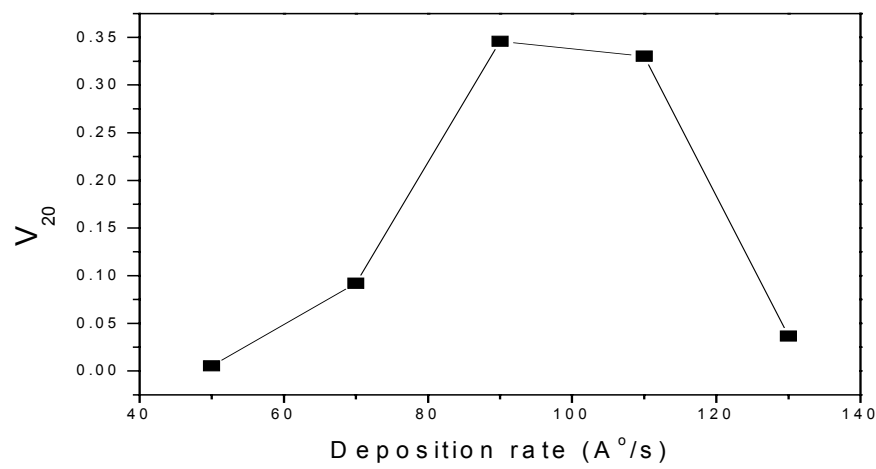

Fig.(3-17) the corrected voltage $\mathrm{V}_{20}$ of the poly-Si:H samples versus of the deposition rate.

For the poly-Si:H samples, the $\tau_{1}$ and $\tau_{3}$ transient could not be observed due to the limited installed time resolution of the apparatus. According to Eq.(2-35) for the stage 2 transient, however, the relaxation time and the initial voltage are interrelated. So that the relaxation time $\tau_{2}$ can be calculated from $V_{20}$ and vice versa as shown in chapter 2 . As a first test for the usefulness of Eq.(2-35), Fig.(3-18) shows the experimental and the calculated relaxation time $\tau_{2}$ of poly-Si:H thin film sample L253. It is shown that the calculated $\tau_{2}$ of the poly-Si:H sample fits to the experimental relaxation time at high temperatures while the fit is not good at low temperatures. However, at least both the experimental and calculated relaxation times $\tau_{2}$ decrease with increasing temperature. As discussed in chapter 2 the difference in $\tau_{2}$ are probably due to the difference in local (internal) and global (source) transient voltage. We can plot also the calculated and experimental relaxation times $\tau_{2}$ as a function of the deposition rate for the poly-Si:H samples at two different fixed temperatures as seen in Fig.(3-19). Again, the experimental and calculated relaxation times fit at the high temperatures while the fit is not so good at low temperatures. However, the trend remains the same. 


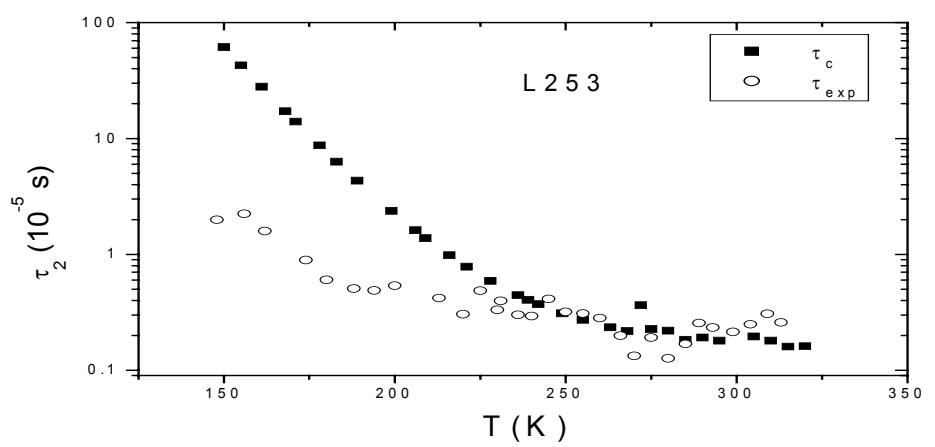

Fig.(3-18) The experimental and calculated relaxation times $\tau_{2}$ for poly-Si:H sample L253 versus the temperature.
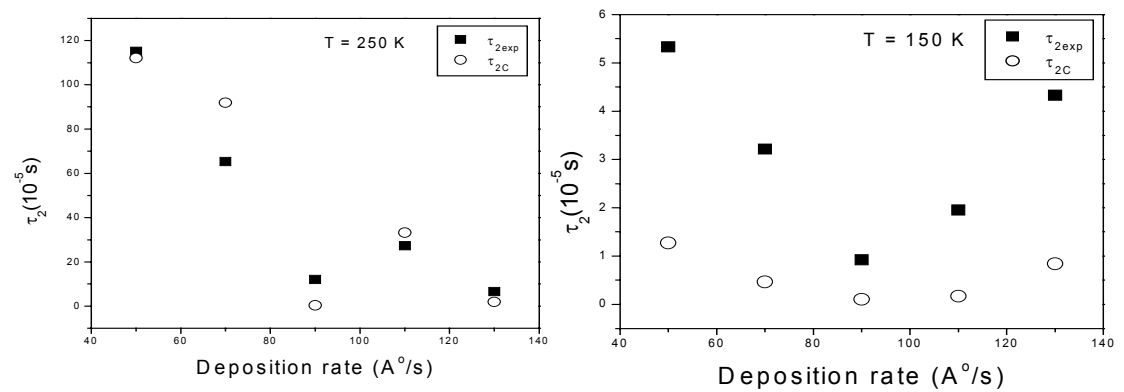

Fig.(3-19) The experimental and calculated relaxation times $\tau_{2}$ for poly-Si:H sample versus the deposition rate.

In chapter 2 it is also suggested that the weighted trap state density difference $\left(\mathrm{N}_{\mathrm{T}}{ }^{+}-\mathrm{N}_{\mathrm{T}}{ }^{-}\right)$can be extracted from $\mathrm{V}_{20}$. For the poly-Si:H sample L252 this is shown in Fig.(3-20). The structure at low energies is stronger than in an n-doped c-Si (Fig.(2-10)). Apparently there is some band tailing at very low energies (measured from the conduction or valence band energies). Furthermore, a trap level appears at $\approx 34 \mathrm{meV}$. As $2 \mathrm{kT}$ is rather can arbitrary integration limit, this value has to be calibrated. For that we use the doping level of phosphorus in an n-doped c-Si sample (upper scale in Fig.(3-20)). Fig.(3-21) shows the maximum trap level ( the trap state density difference at the peak value) and the width of both doping and tailing peaks of the poly-Si:H samples as a function of the deposition rate. The maximum trap level and the width of both doping and tailing peaks have a minimum value at the deposition rate $\approx 90(\AA / \mathrm{s})$ and increase when the deposition rate goes away from this value, again similar to the photomixing results. 


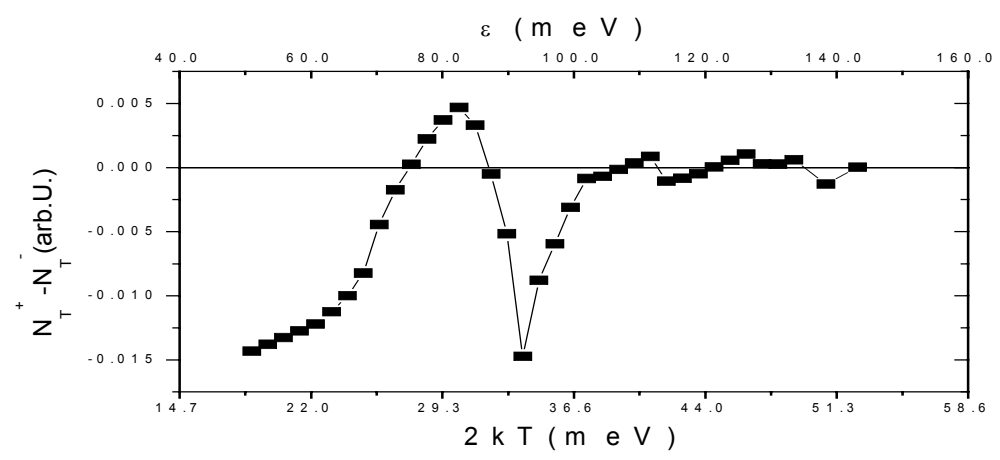

Fig.(3-20) Trap state density difference of the poly-Si:H sample L252 versus $2 \mathrm{kT}$ and $\varepsilon$.
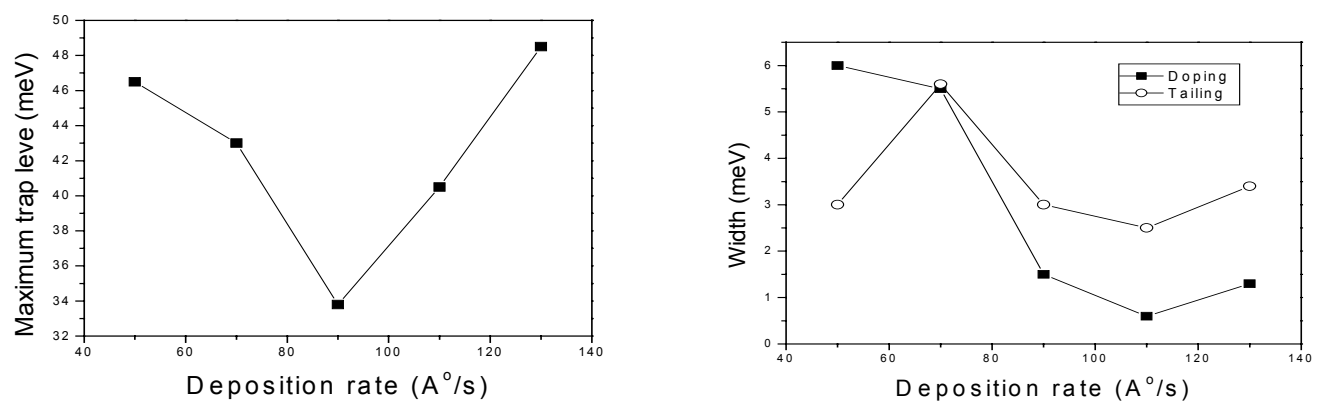

Fig.(3-21) The maximum trap level and the width of both doping and tailing peaks of the poly-Si:H samples versus the deposition rate.

\subsubsection{The hot wire chemical vapor deposition a-SiGe:H samples.}

The transient thermoelectric effects were also measured for the a-SiGe:H films. An example is shown in Fig.(3-22). Unexpectedly, the TTE-voltage of stage 2 decomposes into two transient peaks that are marked 1 and 2 . The transient peak 1 has a larger amplitude and a faster decay than peak 2.

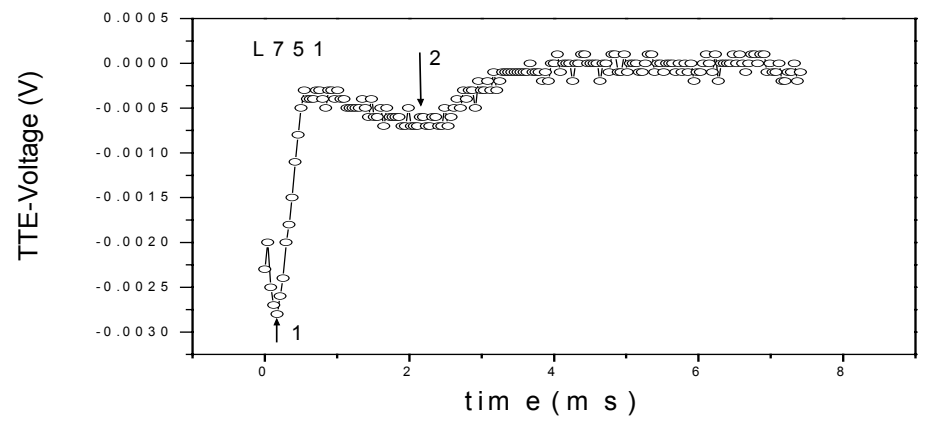

Fig.(3-22) The TTE-voltage of stage 2 of the a-SiGe:H sample L751 versus time. 
The initial transient voltage can be corrected as discussed in chapter 2. Fig. (3-23) shows the calculated voltage of stage 2 of a-SiGe:H samples for each peak as a function of the Tauc gap. Both corrected voltages increase with increasing Tauc gap. Fig.(3-24) show the direct correlation between the two calculated voltages $\mathrm{V}_{2 \mathrm{C}}(1)$ and $\mathrm{V}_{2 \mathrm{C}}(2)$. It is clear that there is a good correlation between the two calculated transient voltages $\mathrm{V}_{2 \mathrm{C}}(1)$ and $\mathrm{V}_{2 \mathrm{C}}(2)$. In contrast, the correlation between the calculated voltages $\mathrm{V}_{2 \mathrm{C}}(1)$ and $\mathrm{V}_{2 \mathrm{C}}$ (2) and Tauc gap is poor, suggesting that we have an interaction between gap states at the same side of the gap; but also that the trap density generally increases with the optical gap as before.
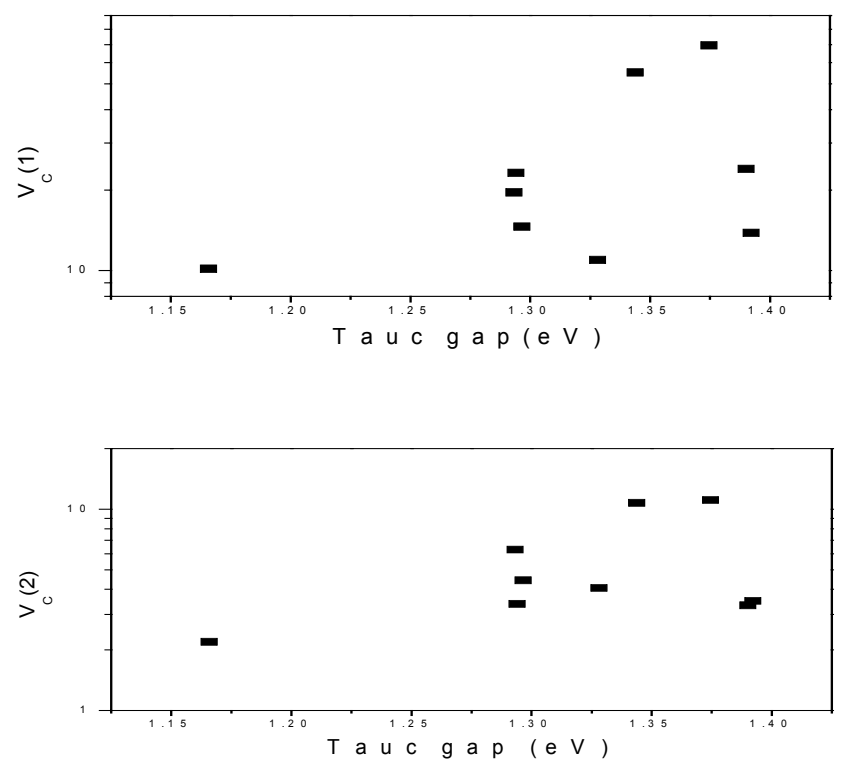

Fig.(3-23) the calculated voltage of stage 2 for a-SiGe:H samples of peaks 1 and 2 versus the Tauc gap.

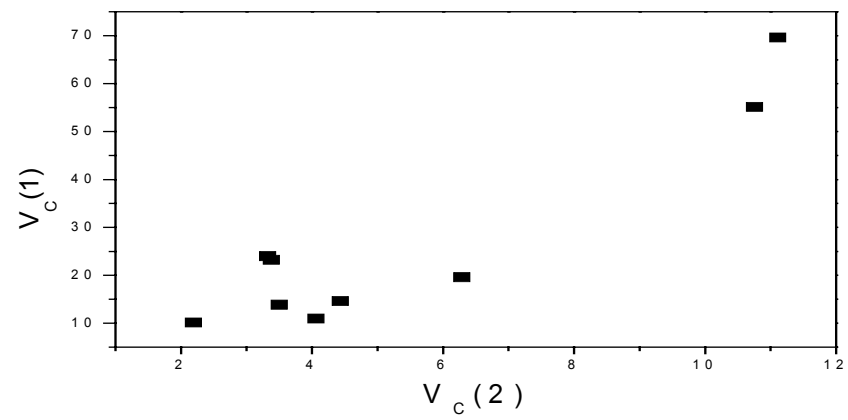

Fig.(3-24) the calculated $V_{C}(1)$ versus the calculated $V_{C}(2)$ for the a-SiGe:H samples. 
Fig.(3-25) shows the experimental and the calculated relaxation time $\tau_{2}$ of aSiGe:H thin film sample L749 for the two peaks 1 and 2. It is shown that the calculated $\tau_{2}$ of the a-SiGe:H sample for each peak fits to the experimental relaxation time at relative high temperature while the fit is not good at low temperature as before for poly-Si:H. However, the experimental and calculated relaxation times $\tau_{2}$ both decrease with increasing temperature and show similar weak structures. We can plot also the relation between both calculated and experimental relaxation time $\tau_{2}$ as a function of the Tauc gap for the a-SiGe:H samples at fixed temperature for the peak 1; as an example; as seen in Fig.(3-26). The experimental and calculated relaxation times trend to fit better at the high temperatures while the fit is not so good at low temperatures both for peak 1 and peak 2 . We have plotted the correlation between the $\mathrm{V}_{2 \mathrm{C}}(1)$ and $\mathrm{V}_{2 \mathrm{C}}(2)$ and find a better correlation also between $\tau_{2}(1)$ and $\tau_{2}(2)$; as shown in Fig.(3-27); that confirms the conclusion obtained from the $\mathrm{V}_{2 \mathrm{C}}(1)$ and $\mathrm{V}_{2 \mathrm{C}}(2)$ correlation.
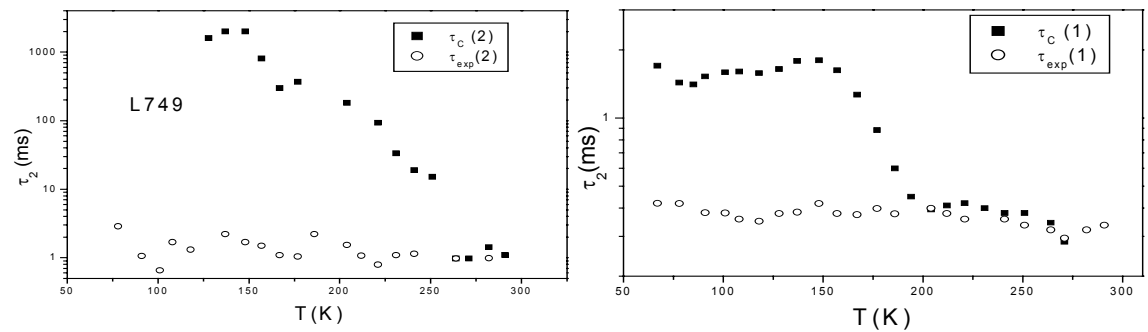

Fig.(3-25) the experimental and calculated relaxation times $\tau_{2}$ of a-SiGe:H sample L749 for the two peaks 1 and 2 versus the temperature.

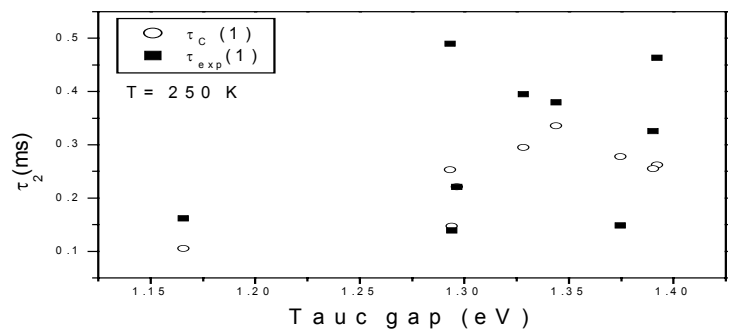

Fig.(3-26) the experimental and calculated relaxation times $\tau_{2}$ of a-SiGe:H samples for the peaks 1 versus the Tauc gap. 


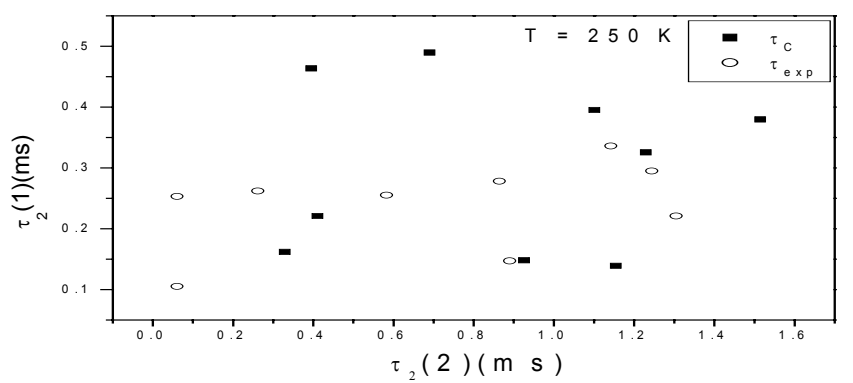

Fig.(3-27) The experimental and calculated relaxation times of peak $1\left(\tau_{2}(1)\right)$ versus $\left(\tau_{2}(2)\right)$ of peak 2 .

Fig.(3-28) shows the trap state density difference for a-SiGe:H sample L740 as extracted from the $\mathrm{V}_{2 \mathrm{C}}$-voltage of each transient peak (1 and 2) as a function of $2 \mathrm{kT}$. Two peaks appear in both trap state differences and they are located at $\approx 15 \mathrm{meV}$ and at $\approx 20$ meV uncalibrated, respectively, that confirms again the correlation between the two voltages. The first peak (at $\approx 15 \mathrm{meV}$ ) has larger amplitude than the second (at $\approx 20$ $\mathrm{meV}$ ). The two trap state density difference peaks that are extracted from the two voltages $\mathrm{V}_{2 \mathrm{C}}(1)$ and $\mathrm{V}_{2 \mathrm{C}}(2)$ have the same shape and the same position but differ in amplitude, suggesting that both transients provide a measure for the total density of trap states, while details of the capture and reemission vary.
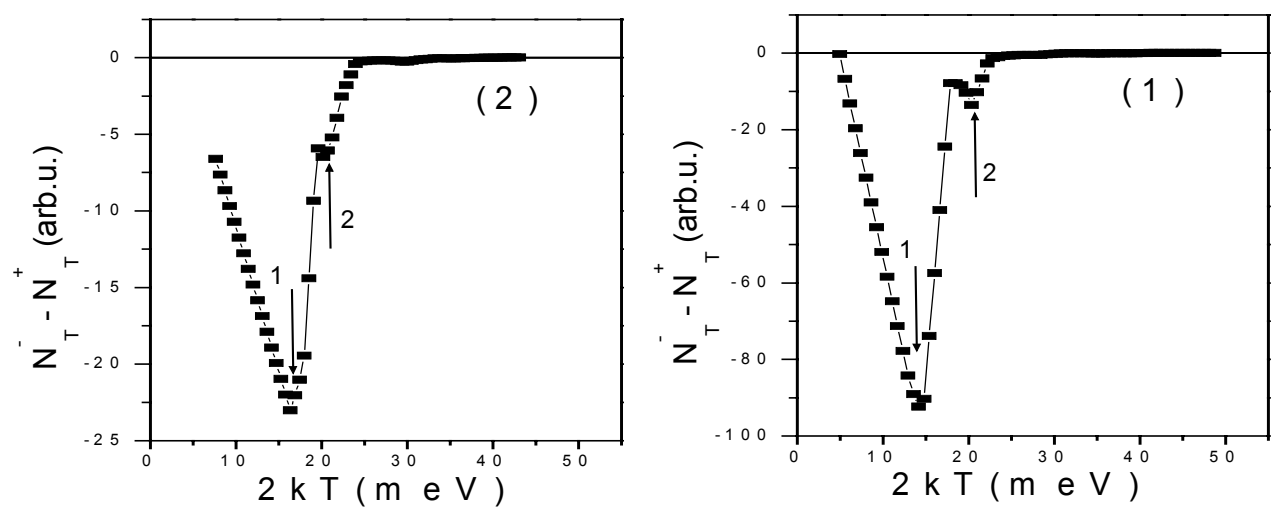

Fig.(3-28) The trap state density difference for a-SiGe:H sample L740 from the transient peaks ( 1 and 2 ) versus $2 \mathrm{kT}$.

The trap state density difference maximum position as a function of the Tauc gap for a-SiGe:H samples for the two peaks and from the two voltage peaks shown in Fig.(3- 
29). Fig. (3-30) shows the correlation between the maximum trap level and the negative peak value of both $V_{2 C}(1)$ and $V_{2 C}(2)$ for the two trap peaks. There is a rather good correlation between them. Again, the correlation with the Tauc gap is not very convincing as compared to the internal correlation. In particular aside the large scatter, the tendency for (1) is opposite to that of (2), suggesting that defects (1) is preferentially installed with higher gap compounds. Fig.(3-31) Show the width of the two voltages peaks as a function of the Tauc gap. The width of two peaks has the same trend like the charge defected density which obtained from the photomixing.
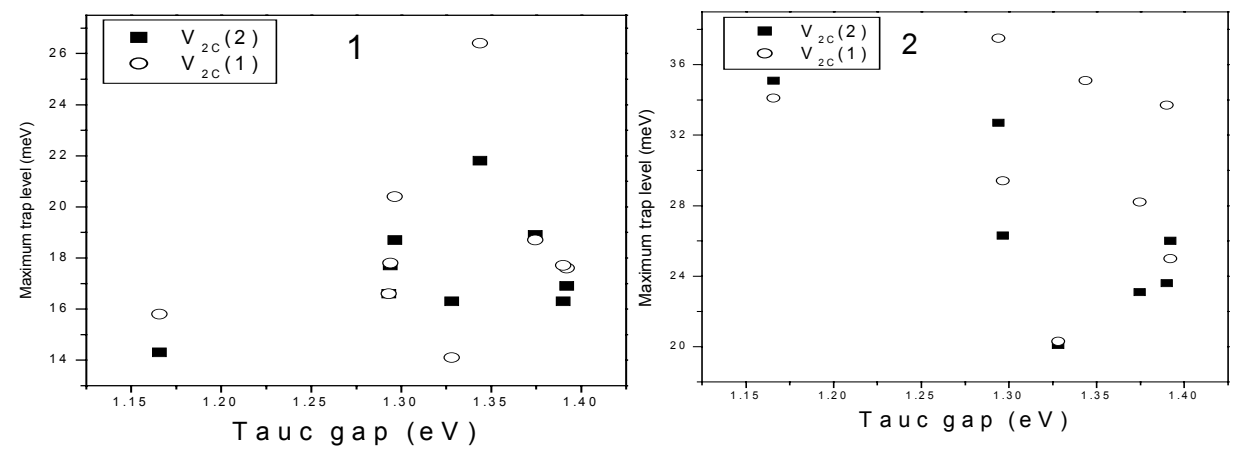

Fig.(3-29) the trap density difference position for a-SiGe:H samples from the two voltage peaks $\mathrm{V}_{2 \mathrm{C}}(1)$ and $\mathrm{V}_{2 \mathrm{C}}(2)$ and from the two peaks of the trap state density difference peaks 1 and 2 versus Tauc gap.
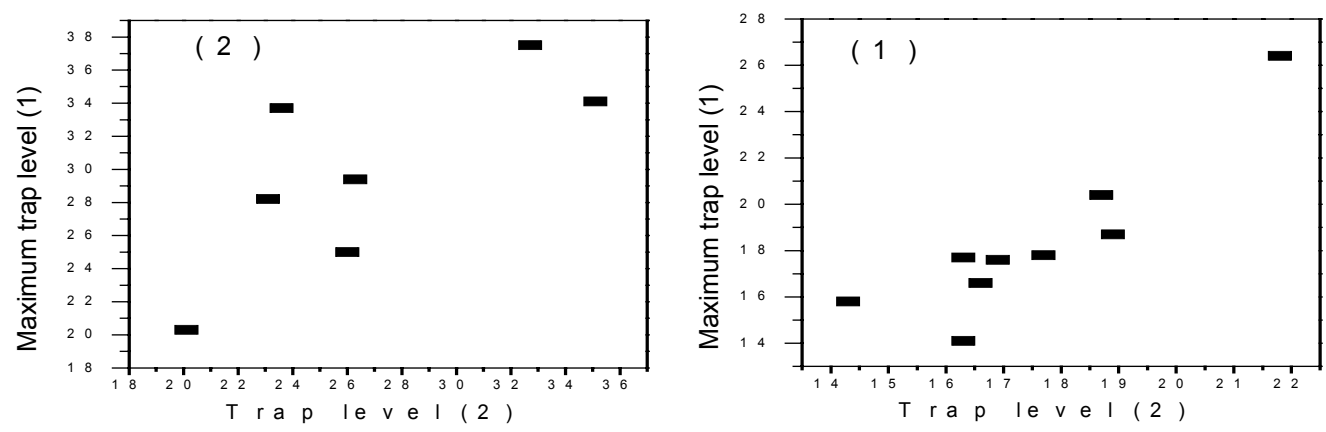

Fig.(3-30) the negative peak value of $\mathrm{V}_{2 \mathrm{C}}(1)$ and the negative peak value of $\mathrm{V}_{2 \mathrm{C}}(2)$ for the two peaks both versus the energy level at the maximum. 


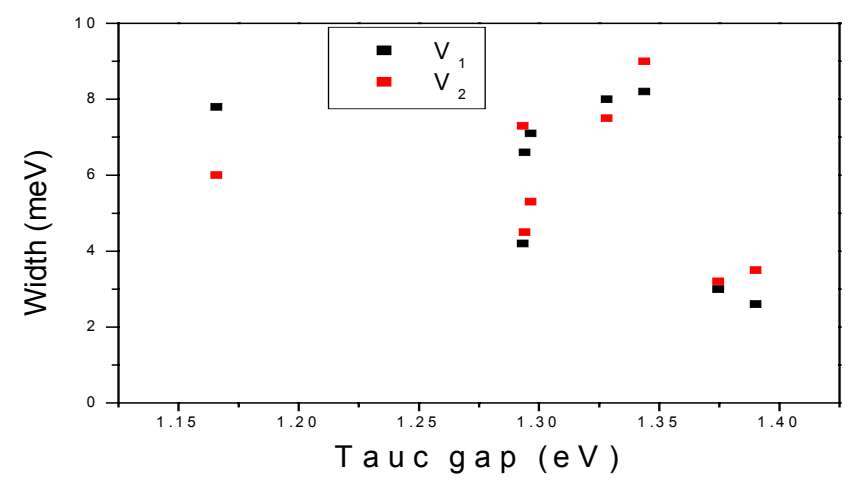

Fig.(3-31) the width of the two voltages peaks $V_{2 C}(1)$ and $V_{2 C}(2)$ versus the Tauc gap for a-SiGe:H films.

\subsubsection{The low pressure reactive ECR plasma $\mu \mathrm{c}-\mathrm{SiGe:H}$ samples}

The thermoelectric effect signal of the $\mu \mathrm{c}-\mathrm{SiGe}: \mathrm{H}$ samples is very similar to that of a-SiGe:H samples; i.e. there are two consecutive voltage peaks (1) and (2). However, we can find the two peaks only at the lowest temperatures. We could not resolve the time constant of the first transient peak, however, we can determine the initial transient voltage. Here, the transient voltage changes its sign with the temperature similar to that of the n-doped c-Si [88] as shown in Fig.(3-32). Fig. (3-33) shows the transient voltage of the $\mu \mathrm{C}-\mathrm{SiGe}: \mathrm{H}$ sample $\mathrm{SiGe} 2 / 5995$ as a function of the temperature. The initial transient voltage $\mathrm{V}_{20}$ has two relative minima, one at $157 \mathrm{~K}$ and the other at $222 \mathrm{~K}$ similar to the a-SiGe:H samples. From the shapes following the two minima we can determine two trap states directly as was done for the n-doped c-Si sample and the a-SiGe:H samples. Fig.(3-34) shows the trap state density difference $\left(\mathrm{N}_{\mathrm{T}}{ }^{+}-\mathrm{N}_{\mathrm{T}}{ }^{-}\right)$as a function of the $2 \mathrm{kT}$. The two trap state density difference levels are located at $22.5 \mathrm{meV}$ and $35.5 \mathrm{meV}$ (uncalibrated); these are almost the same level values as found for the a-SiGe:H samples suggesting alloy randomness as the cause. Fig.(3-35) shows the two trap levels and the its width as a function of the deposition rate. The width of the doping level have the same trend like the charge defect density obtained from photomixing. The two trap levels have minimum values at the deposition rate $\approx 0.8 \AA / \mathrm{s}$ similar to the photomixing results. 

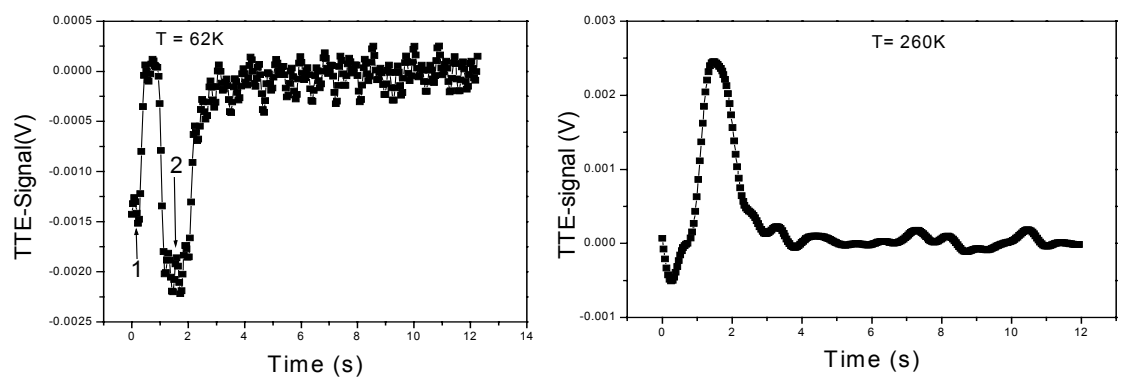

Fig.(3-32) The TTE-transient of the $\mu \mathrm{c}-\mathrm{SiGe}: \mathrm{H}$ sample at $\mathrm{T}=62 \mathrm{~K}$ and $\mathrm{T}=260 \mathrm{~K}$.

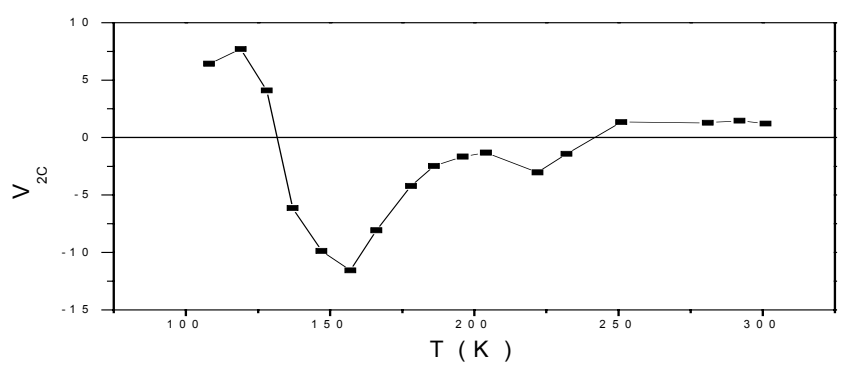

Fig.(3-33) The calculated transient voltage of the $\mu \mathrm{c}-\mathrm{SiGe:H}$ sample 2/5995 versus the temperature.

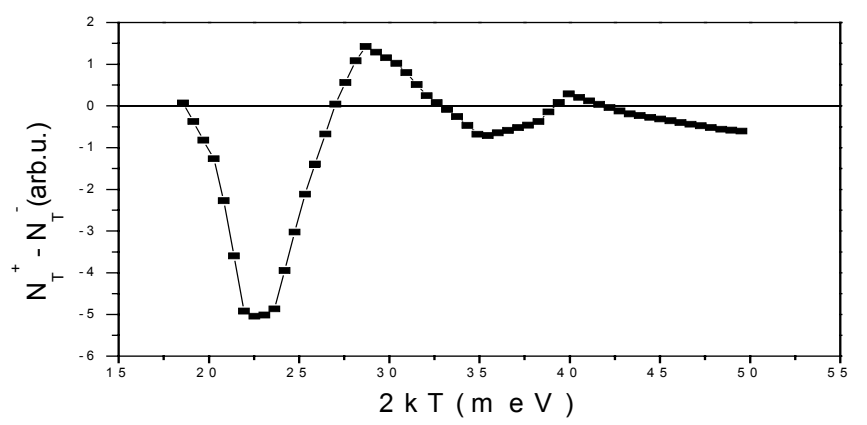

Fig.(3-34) The trap state density difference of the $\mu \mathrm{c}-\mathrm{SiGe}: \mathrm{H}$ sample 2/5995 versus 2kT. 

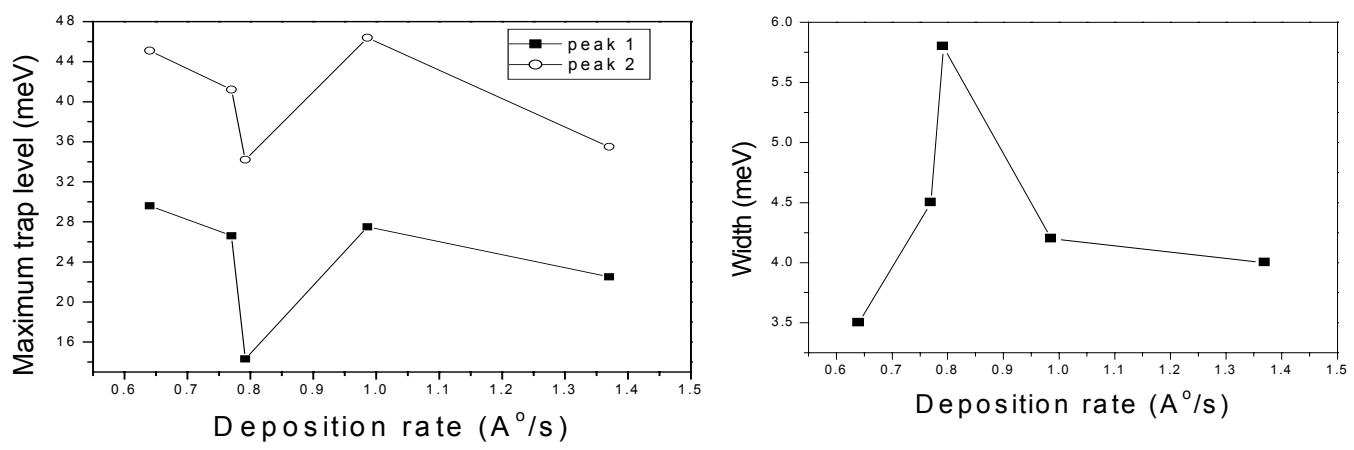

Fig.(3-35) the maximum trap level of the $\mu \mathrm{c}-\mathrm{SiGe}: \mathrm{H}$ samples for the two peaks versus the deposition rate.

The relaxation time of the $\mu \mathrm{c}-\mathrm{SiGe}: \mathrm{H}$ samples $\tau_{2}$ can be obtained from the transient signal and also can be calculated from the relaxation transient voltage $V_{20}$ according to Eq.(2-35). The experimental and calculated relaxation times $\tau_{2}$ of the $\mu \mathrm{c}-$ SiGe:H sample (2/5995) are shown as a function of the temperature in Fig.(3-36). They coincide better for the positive voltage as for the negative ones. Fig.(3-37) shows both times $\tau_{2}$ at $\mathrm{T}=250 \mathrm{~K}$ as a function of the deposition rate. There is no particular structure found here which would favor a certain deposition rate, probably the Si-Ge random disorder is dominating here.

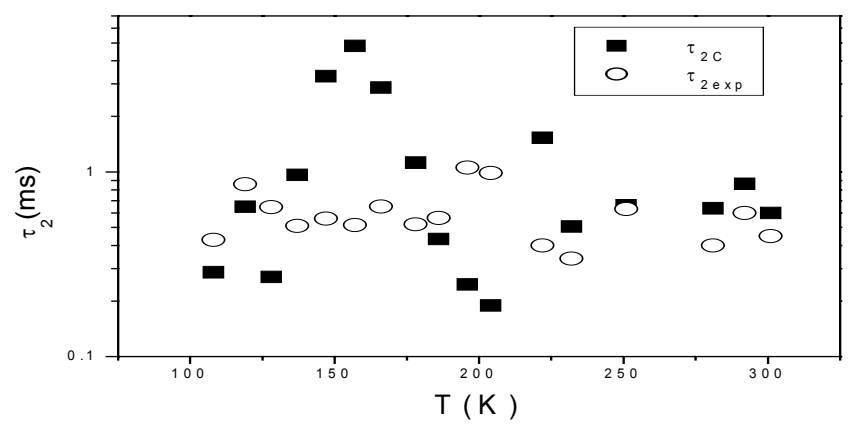

Fig.(3-36) the experimental and calculated relaxation time $\tau_{2}$ of the $\mu \mathrm{c}-\mathrm{SiGe}: \mathrm{H}$ sample 2/5995 versus the temperature. 


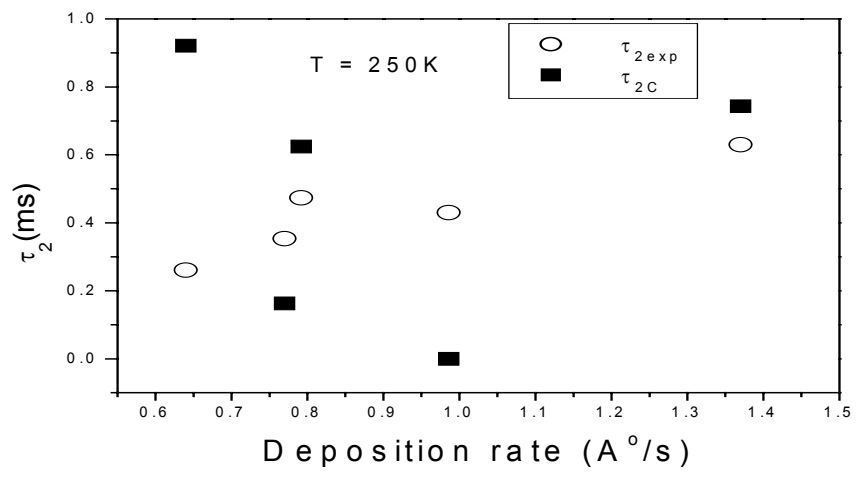

Fig.(3-37) the experimental and calculated relaxation time $\tau_{2}$ of the $\mu \mathrm{c}-\mathrm{SiGe}: \mathrm{H}$ samples at $\mathrm{T}=250 \mathrm{~K}$ versus the deposition rate.

\subsection{Photoluminescence and small angle X-ray spectroscopy (SAXS)}

In order to correlate the transport properties of the HWCVD a-SiGe:H with the microstructure in the films, we also performed photoluminescence and small angle X-ray scattering (SAXS) measurements. In general it is assumed that the Photoluminescence (PL) peak energy position (at $80 \mathrm{~K}$ ) is correlated to the optical gap and that the relative efficiency is inversely proportional to the density of defects. In Fig.(3-38a) we show the relative photoluminescence intensity as function of photon energy at $80 \mathrm{~K}$ for samples L743, L744, and L745. The peak intensity as obtained from this figure is summarized together with key deposition data in Table (3-1). In order to compare the photoluminescence spectral line shape, the normalized photoluminescence spectra are plotted in Fig.(3-38b). First, we get the photoluminescence peak energy positions (Table (3-1)). They correlate with the optical gap as expected.

The relative photoluminescence intensity of sample L744 is only about $30 \%$ less than that of sample L743, whereas the photoluminescence peak energy decreased about $0.1 \mathrm{eV}$. The higher Ge content of L743 reduces the optical gap, with a small increase in the defect density, relative to L744. However, L745 has an order of magnitude larger defect density than L744. L745 was grown by reducing both the filament and substrate temperatures as compared to the making of L744. We light soaked all three samples with an in-situ $100 \mathrm{~mW} / \mathrm{cm}^{2}$ white light for 2 hours. There were no obvious changes of the photoluminescence peak spectra before and after light soaking of samples L743 and 
L744. There was a $30 \%$ increase of the photoluminescence peak relative intensity for sample L745 as is shown in Fig.(3-39). Usually the photoluminescence peak intensity at $80 \mathrm{~K}$ has no obviously change, at best a slight decrease due to metastable defect creation. However, if both structural changes and defect creation takes place in a nonhomogeneous film, the Photoluminescence peak intensity could increase, as is observed [98].
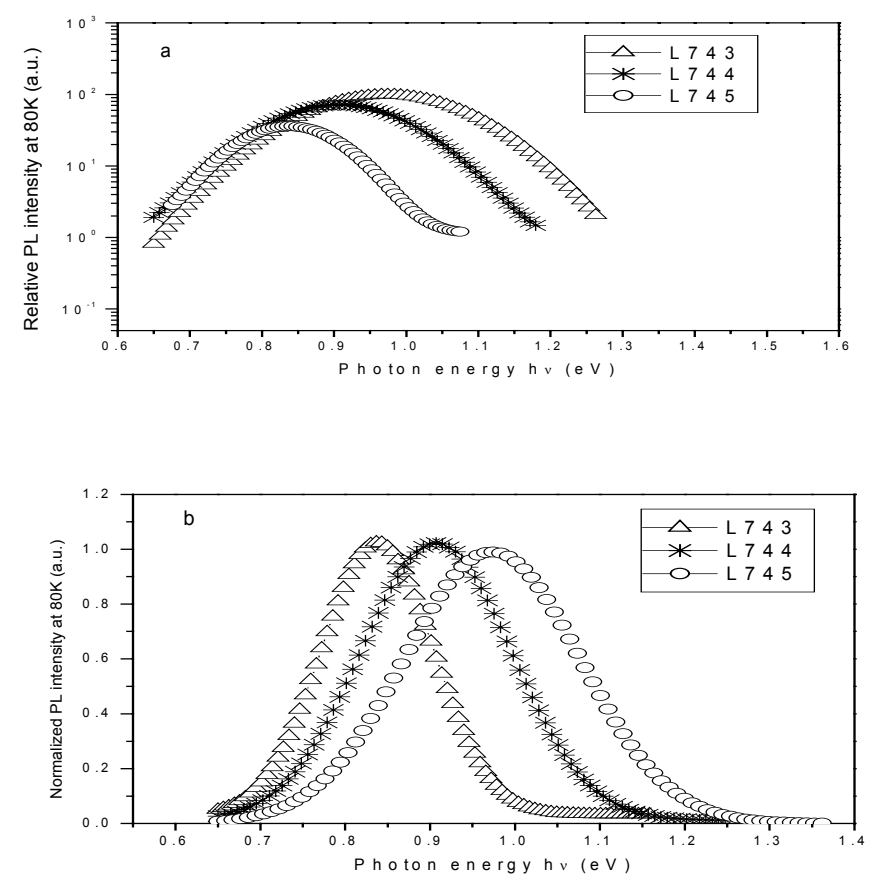

Fig.(3-38) Photoluminescence peak spectra at $80 \mathrm{~K}$ for samples L743, L744, and L745, (a) relative intensity and (b) normalized spectra.

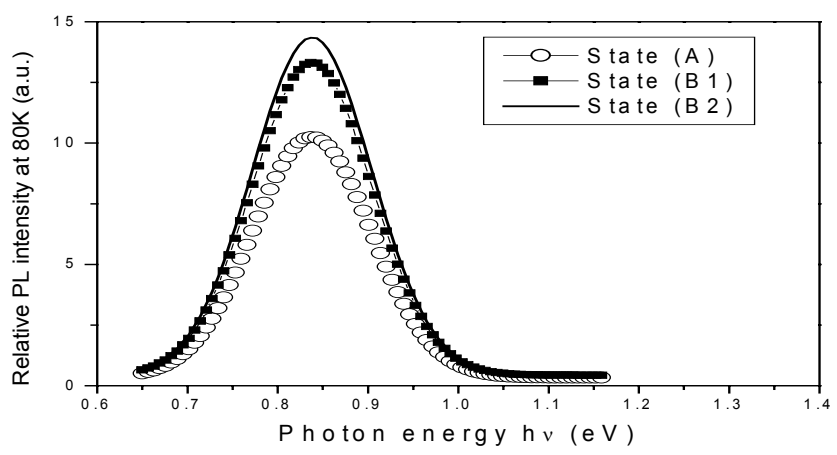

Fig.(3-39) Light-induced effects on PL spectra for sample L745. The photoluminescence peak intensity increased about $30 \%$ from state (A) after light soaking by a $100 \mathrm{~mW} / \mathrm{cm}^{2}$ 
white light for 2 hours (state B1). There is only a small additional change with an additional 2 hours of light soaking (state B2).

In Fig.(3-40) we show SAXS scans for a set of alloys grown under conditions similar to those discussed above (Table (3-2)). There is a systematic increase in the SAXS intensity, $Q_{N}$, with increasing Ge content of the film, with the exception of L828, which has the smallest intensity of this set. In Fig.(3-41) we show the integrated SAXS intensity $Q_{N}$ as a function of the Tauc gap for each sample (top frame). $Q_{N}$ is a measure of the total electron density fluctuations within the film [109] and correlates to nonuniformity of the film due to nanovoids or Ge concentration fluctuations [110]. An increase in $\mathrm{Q}_{\mathrm{N}}$ implies an increase in the nanostructural heterogeneity, and hence, an increase in either the void density or the Ge non-uniformity, or both. We know from previous work (dashed line), that the nanostructural heterogeneity displays a sharp increase at bandgaps $\leq 1.5 \mathrm{eV}$. The current samples have bandgaps below this transition and hence we expect and indeed have large $\mathrm{Q}_{\mathrm{N}}$. We model the SAXS data using spherical objects of an average diameter $\langle\mathrm{D}>$ as the cause of the heterogeneity in the films [109]. The values for $<\mathrm{D}>$ for these films are given in Table (3-2) and are only somewhat smaller than the range of the LRPF as deduced from the photomixing technique (Fig. 3-11).

In the middle frame of Fig.(3-41) we see that this set of a-SiGe:H films has a photoresponse similar to our narrow gap alloys grown at higher deposition rates (line), except for L828 which has a very high value for the gap $(1.25 \mathrm{eV})$. In the bottom frame of Fig.(3-41) we see that the Urbach energy $\left(E_{U}\right)$ as measured by the constant photocurrent method (CPM) shows a systematic increase with decreasing Tauc gap, again with the exception of $\mathrm{L} 828$, which has a lower $\mathrm{E}_{\mathrm{U}}$ than the trend. The anomalous sampleL828-was grown with a lower substrate temperature $\left(150^{\circ} \mathrm{C}\right)$ and a lower filament temperature $\left(1800^{\circ} \mathrm{C}\right)$ than the others. 


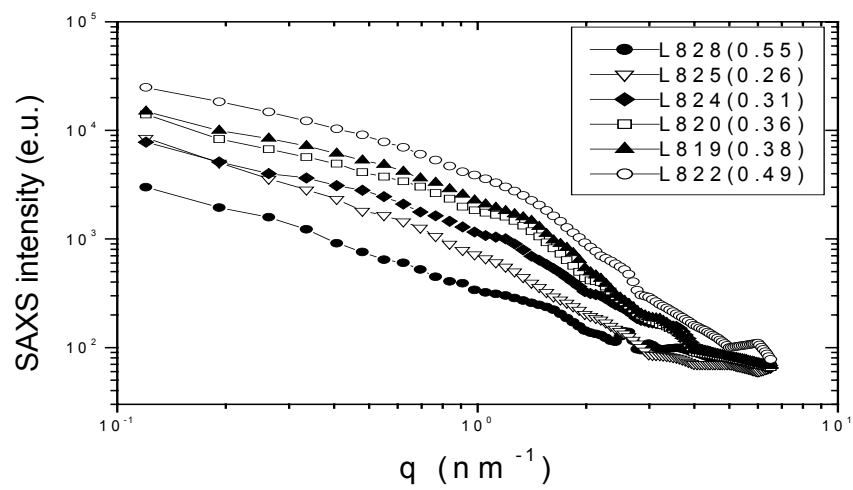

Fig.(3-40): SAXS intensity for a-Si $i_{1-x} \mathrm{Ge}_{\mathrm{x}}$ : $\mathrm{H}$ alloys with various $\mathrm{x}$ the values of which are given in parenthesis.

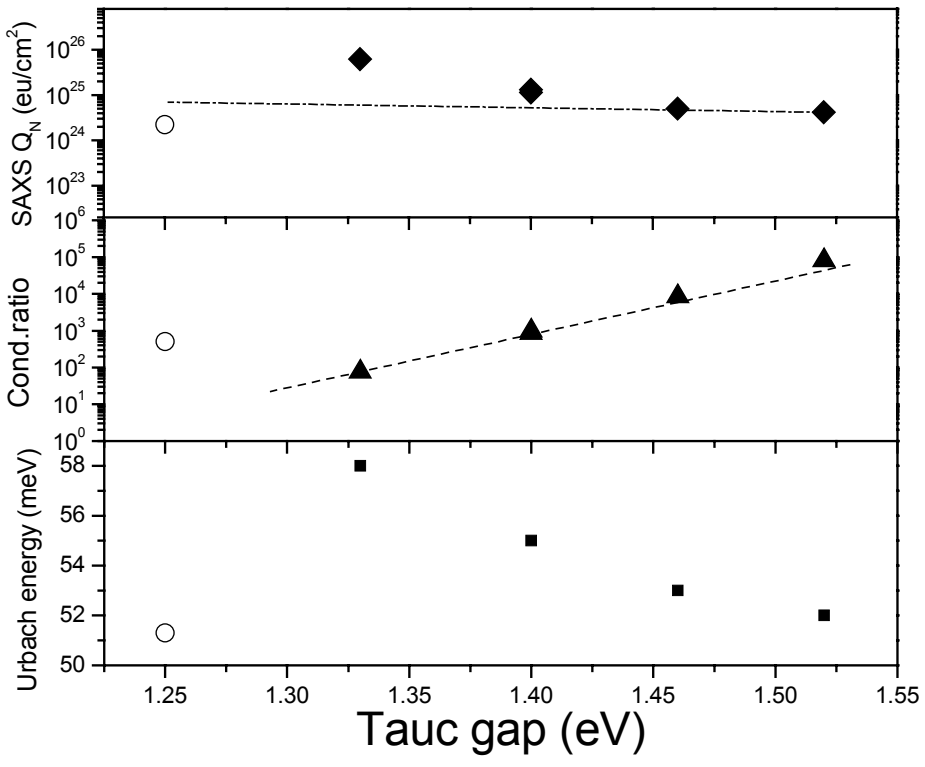

Fig.(3-41): The integrated SAXS intensity (top frame), photoresponse (mid frame) and Urbach energy (bottom frame) as a function of the Tauc gap. The dashed lines are the best data from reference [111]. 
Table (3-1) deposition and PL summary data. All samples were grown with a 1:1 $\mathrm{H}_{2}$ dilution $(25 \mathrm{sccm})$

\begin{tabular}{|l|l|l|l|}
\hline Sample & L743 & L744 & L745 \\
\hline \hline Heater T $\left({ }^{\circ} \mathrm{C}\right)$ & 250 & 250 & 125 \\
\hline $\mathrm{SiH}_{4} /\left(\mathrm{SiH}_{4}+\mathrm{GeH}_{4}\right)$ & 25 & 35 & 35 \\
\hline Filament $\mathrm{T}\left({ }^{\circ} \mathrm{C}\right)$ & 2150 & 2150 & 1800 \\
\hline Tauc gap $(\mathrm{eV})$ & 1.37 & 1.29 & 1.17 \\
\hline PL intensity & 550 & 400 & 22 \\
\hline PL peak E position $(\mathrm{eV})$ & 0.98 & 0.89 & 0.82 \\
\hline
\end{tabular}

Table (3-2) deposition, SAXS, and CPM summary data. All samples were grown with a $1: 1 \mathrm{H}_{2}$ dilution $(25 \mathrm{sccm})$

\begin{tabular}{|c|c|c|c|c|c|c|}
\hline Sample & L825 & L824 & L820 & L819 & L822 & L828 \\
\hline Heater T $\left({ }^{\circ} \mathrm{C}\right)$ & 250 & 250 & 250 & 250 & 250 & 150 \\
\hline $\mathrm{SiH}_{4} /\left(\mathrm{SiH}_{4}+\mathrm{GeH}_{4}\right)$ & 0.13 & 0.18 & 0.25 & 0.25 & 0.35 & 0.13 \\
\hline Filament T $\left({ }^{\circ} \mathrm{C}\right)$ & 2100 & 2100 & 2100 & 2100 & 2100 & 1800 \\
\hline Tauc gap $(\mathrm{eV})$ & 1.52 & 1.46 & 1.40 & 1.40 & 1.33 & 1.25 \\
\hline $\mathrm{Ge}($ at. $\%)$ by SIMS & 25.5 & 30.6 & 35.9 & 37.6 & 49.1 & 55.3 \\
\hline $\mathrm{Q}_{\mathrm{N}}\left(10^{24}\right.$ eu/cm $\left.{ }^{3}\right)$ by SAXS & 3.25 & 6.26 & 9.01 & 11.5 & 19.7 & 1.86 \\
\hline$<\mathrm{D}>(\mathrm{nm})$ by SAXS & 4.1 & 2.9 & 3.4 & 3.5 & 3.2 & 3.1 \\
\hline $\mathrm{E}_{\mathrm{u}}(\mathrm{meV})$ by CPM & 51 & & 52 & & & 53 \\
\hline
\end{tabular}




\section{Discussion}

\subsection{Transport properties of poly-Si:H thin films}

The poly-Si:H thin film material is one of the wide application materials in the solar technology. It is important to be able to prepare these materials on a large scale and to optimize the preparation conditions. The deposition rate of these materials is the main parameter which affects the large-scale production; besides there are other preparation parameters such as the substrate temperature, gas pressure and size. The photomixing technique was employed to measure the charge transport properties of the poly-Si:H films and they indeed change very significantly with the deposition rate.

\subsubsection{Transport properties of the hot wire chemical vapor deposition poly-Si:H samples}

There are three poly-Si:H samples states that are: state (A): is the as-deposited samples, state (B): is the low temperature annealed samples, at $50-70 \mathrm{~K}$ for 24 hours, and state $(\mathrm{C})$ is the recovered samples after low temperature annealing by annealing again at $150^{\circ} \mathrm{C}$ for 2 hours. In this context we will discuss the charge transport properties of these three states of HWCVD poly-Si:H as a function of the deposition rate.

\section{Transport in as-deposited samples (A)}

The effect of the deposition rate on the hot wire chemical vapor deposited poly$\mathrm{Si}: \mathrm{H}$ thin films and its charge transport properties is quite large. As seen in Figs. $(3-1,2,3)$, the photoconductivity, drift mobility and lifetime of as-deposited poly-Si:H thin films have a peak at the deposition rate $\approx 90 \AA / \mathrm{s}$. This means that the poly-Si:H thin film material has the best charge transport properties at this deposition rate. This means also, that the deposition rate and the other deposition parameters such as the silane flow rate, hydrogen dilution, substrate temperature; filament temperature, filament-substrate distance etc. can be optimized to prepare a high quality film. Note also, that the charge transport parameters of the hot wire chemical vapor as-deposited poly-Si:H film fall off rather sharply on both sides of the optimal deposition rate (at $\approx 90 \AA / \mathrm{s}$ ). This means that one has to monitor the deposition rate rather precisely. It has been speculated that the 
decay of the charge transport properties may be due to an increasing grain boundary area [112]. The properties of the boundaries between adjacent grains (as opposed to the properties of the single grains themselves) are indeed most important in understanding the electrical properties of the film. Atomic level disorder at the boundaries between grains introduces defect states that may be filled with trapped charge. The trapped charge then causes energy band bending and this introduces energy barriers for the charge transport. The trapped charge can form certain potential barriers and with it the longrange potential fluctuations. In our samples, the potential fluctuations were determined from the mobility increase with increasing applied electric field as shown in Fig.(3-4). From this type of data we have calculated the length and depth of the long- range potential fluctuations (Figs. (3-4,5)). As expected, the minimal depth of the potentials occurs in the region where the mobility and lifetime peak. When the deposition rate goes away from the peak value $(\approx 90 \AA / \mathrm{s})$ the depth increases, indicating enhanced potential wells. In addition, the relative charge density is increased too.

We now give our attention to the effect of the deposition rate on the resultant film properties. Since the high deposition rates were achieved by increasing deposition pressure, silane flow rate, and decreasing filament- to- substrate distance, there may exist significant interactions among these parameters.

From table (2-1), we can see that the deposition rate increases monotonically with increasing deposition pressure and silane flow rate. In contrast, the substrate temperature does not have a significant influence on the deposition rate. Already at $316^{\circ} \mathrm{C}$ substrate temperature, high quality films can be grown. Obviously low pressure, medium silane flow rate, and a $316{ }^{\circ} \mathrm{C}$ substrate temperature are generally enough to maintain high quality poly-Si:H thin film at a high deposition rate.

\section{Transport in the annealed samples}

Comparison of the charge transport properties of poly-Si:H films in the annealed state (B) and recovery state (C) is shown in Fig. (3-7). The charge transport properties of films in state (B) are significantly different from that of the as-deposited state (A) and state (C). Specifically, the low temperature annealing treatment state (B) alters the charge transport properties to the worth. In particular, not only the trend of the charge transport 
properties as a function of the deposition rate changes but also the values are lower for samples (B) as for as-deposited samples (A). This may be due to a change in the arrangement and the electrical properties of the grain boundaries. This change in the grain boundaries might go with an increase in the number of trapping states and the potential barriers. From our measurements of the depth of the long- range potential fluctuation, Fig.(3-8), we found that the value of depth increases by a factor of two as compared to that of as-deposited films. Besides, the depth of the potential fluctuations, and the charge defect density increases too. For state (C), the A-type charge transport properties are practically recovered. They have the same trend and almost the same values for the asdeposited samples (A). The depth and length of the long-range potential fluctuations are almost the same, too.

\subsection{Transport properties of the hot wire chemical vapor deposition a-SiGe:H samples}

Shortly after the realization that hydrogenated amorphous silicon (a-Si:H) afforded the promise of an economical photovoltaic technology, investigations began into the alloys of this material, predominantly mixing with $\mathrm{Ge}$ and $\mathrm{C}$, to provide optical gap tenability. The addition of Ge in particular allows one to reduce the gap value from 1.7$1.8 \mathrm{eV}$ for pure a-Si:H continuously down to $1.0 \mathrm{eV}$.

In Figs. $(3-9,10)$, we show the photoconductivity (which is proportional to the $\mu_{\mathrm{d}} \tau$ product) and both the drift mobility and lifetime as a function of the Tauc gap. In a previous study of a HWCVD a-SiGe:H film, we were unable to obtain a good enough photoresponse from samples with enough Ge in them to lower the bandgap below $1.4 \mathrm{eV}$, and thus were not able to report on measurements using the photomixing technique for such narrow bandgap alloys[113]. After we produced superior a-SiGe:H alloys, while both the drift mobility and lifetime have vastly smaller values than the a-Si:H, they at least measurable. As was suggested before [113], the decrease in drift mobility $\mu_{\mathrm{d}}$ results from an increase in the number of charged scattering centers that can also produce longrange potential fluctuations. The measurements of the electric field dependence of the mobility and lifetime for a-SiGe:H alloys show strong evidence for the presence of long- 
range potential fluctuations. We have calculated the length and depth of the potential fluctuations according to Eq.(3-1) and show the results in Fig.(3-11). As expected, the mobility decreases when the depth of the long-range potential fluctuation increases Fig(41a), while we do not have a good correlation with the length of the long-range potential fluctuations (Fig.(4-1b)). This is because the drift mobility is directly proportional to the $\exp \left(-\mathrm{eV}_{\mathrm{p}} / \mathrm{kT}\right)$ but also is proportional to the product $\mathrm{LE}$, which is smaller than $\mathrm{V}_{\mathrm{p}}$, according to Eq.(3-1). According to Eq.(2-18), the lifetime is inversely proportional to the drift mobility. Then, from Eq.(3-1), the lifetime should have a good correlation with length of the long-range potential fluctuation [Fig.(4-2a)], and no correlation with the depth [Fig.(4-2b)], as is observed. This means that the long-range potential fluctuation model is working and can be used to explain the charge transport properties of our samples.
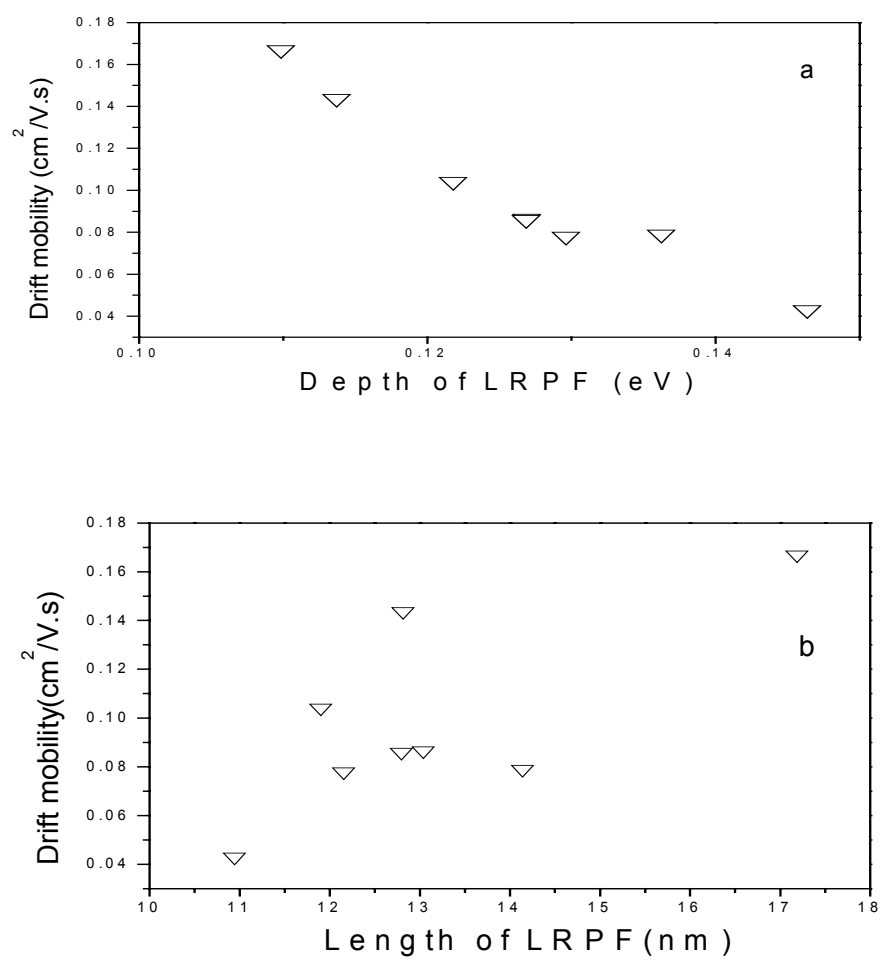

Fig. (4-1) the drift mobility of the a-SiGe:H samples versus the depth (a) and the length (b) of the LRPF. 

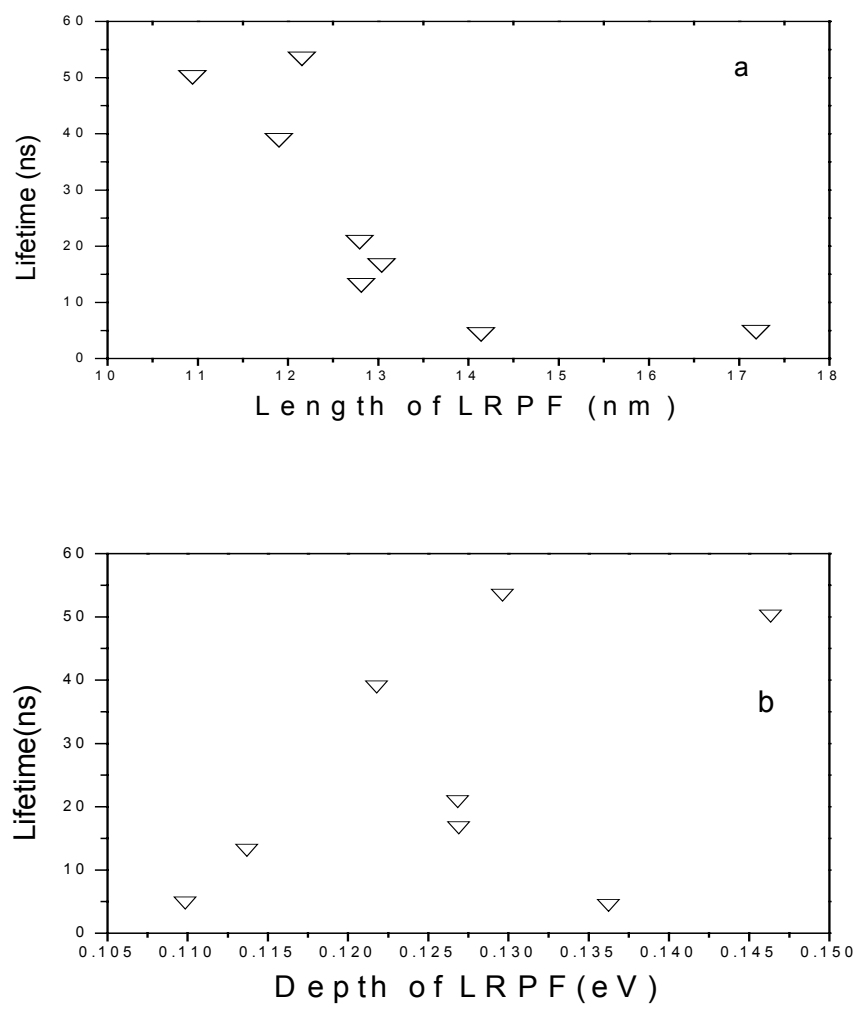

Fig.(4-2) the lifetime of the a-SiGe:H samples versus the length (a) and the depth (b) of the LRPF.

There have been many observations of the deterioration of the opto-electronic properties of the a-SiGe:H alloys with increased Ge content [114-117]. While most prior work has explained such deterioration in term of increased defect density and wider band-tail states (larger Urbach energy), other prior work has mentioned the possibility of a deterioration of the electron mobility upon Ge alloying [118]. In our samples, the deterioration of the transport properties is probably due to the increase of the charge defect density as to be concluded from the photomixing data; Fig.(3-11).

\subsection{Transport properties of the low pressure reactive ECR plasma $\mu c-S i G e: H$ samples.}

In this work we were also interested to measure, for the first time, the charge transport properties of ECR- $\mu \mathrm{c}-\mathrm{SiGe}: \mathrm{H}$ samples. As seen in Fig.(3-12) the photoconductivity has a maximum value at a deposition rate $\approx 0.8 \AA / \mathrm{s}$. However, the 
lifetime has a maximum at the same value of the deposition rate while the drift mobility has a minimum. This means that the lifetime is the dominant parameter concerning the photoconductivity. The peak of the photoconductivity may be due to the improvement of the sample crystallinity, in particular a decrease in the number of grain boundaries. Again, the electric field dependence of the drift mobility and lifetime shows evidence for the presence of the long-range potential fluctuations. As expected, the mobility decreases with increasing depth of the long-range potential fluctuations as seen in Fig(4-3) and the lifetime decreases with increasing length of the long-range potential fluctuations as seen in Fig.(4-4). This means that the long-range potential fluctuation model can be used to explain the charge transport properties here, too. We also calculated the relative charge defect density, which has a peak at the optimal deposition rate $\approx 0.8 \AA / \mathrm{s}$.

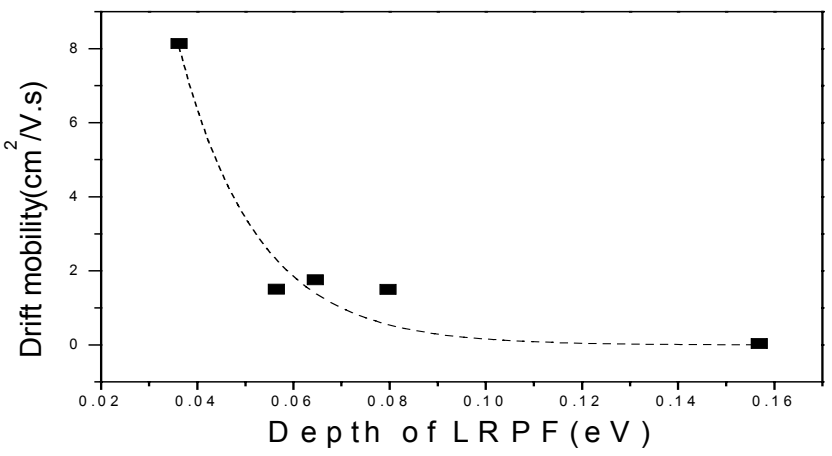

Fig. (4-3) the drift mobility of the $\mu c-S i G e: H$ samples versus the depth of the LRPF. The line is drawn as guide for the eye.

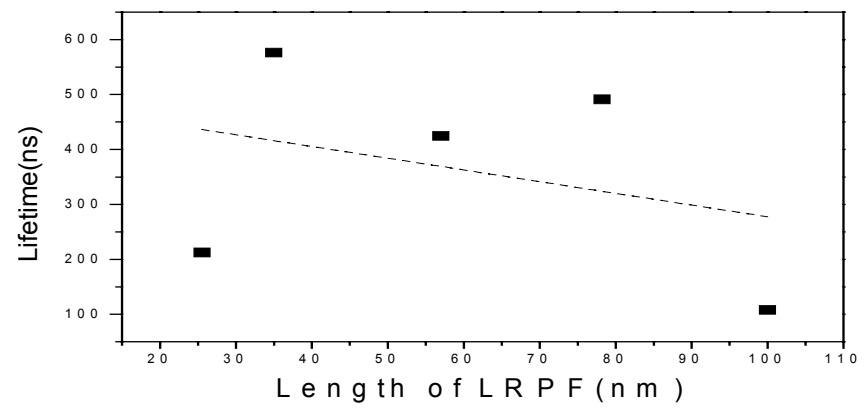

Fig.(4-4) the lifetime of the $\mu \mathrm{c}-\mathrm{SiGe}: \mathrm{H}$ samples versus the length of the LRPF. The line is drawn as guide for the eye. 
Using the growth parameters shown in Table (2-3) we were able to produce microcrystalline $\mathrm{Si} / \mathrm{Ge}$ by the ECR technique. Previously we had used this technique to produce a range of band gaps of amorphous GeSi alloys[120]. The charge transport properties of low pressure reactive ECR plasma $\mu \mathrm{c}-\mathrm{SiGe}: \mathrm{H}$ samples are for the first time measured by the photomixing technique. The transport properties are improved as compared to those of the $\mu \mathrm{c}-\mathrm{SiGe}: \mathrm{H}$ samples which were prepared by VHFPE-CVD[121] and PECVD[122] techniques. Also, if we compare the charge transport properties of $\mu \mathrm{c}-$ SiGe:H samples and the ECR a-SiGe:H samples[120], we find that the drift mobility $\mu \mathrm{c}-$ SiGe:H samples is higher than that of a-SiGe:H samples by two orders of magnitude and the lifetime by one order of magnitude; as expected. This may have important consequences on the thin film photovoltaics field. It is becoming clear that the thin films are getting competition from single crystalline Si solar cells, which shows no decay with the time of illumination. However, microcrystalline semiconductors still have a role, as at the critical compositions between amorphous and crystalline and their transport properties are still good and stable.

\subsection{Space charge voltage of poly-Si:H films}

In chapter2 it has been shown that one can obtain the space charge voltage by two different ways. First, from the initial voltage $V_{20}$ of the transient which, however, has to be corrected for the path resistance and the amplifier impedance $\left(\mathrm{V}^{\mathrm{g}}{ }_{20}\right)$ and second, from the time constant of the space charge transient $\tau_{2}$ using Eq. $(2-57)\left(\mathrm{V}^{\mathrm{L}}{ }_{20}\right)$. From the first calculation, we found that the TTE- voltage $\mathrm{V}^{\mathrm{g}}{ }_{20}$ decreases with increasing temperature; as seen in Fig.(2-14). Also, the local voltage $\mathrm{V}^{\mathrm{L}}{ }_{20}$ decrease with increasing temperature, probably due to the increasing electron (hole) emission from the trap states.

According to Eq.(2-57) we have to take the differential with temperature in order to obtain the weighted trap state density difference. Using the calibration procedure described in chapter 2 we can also plot the weighted difference density of states $\left(\mathrm{N}_{\mathrm{T}}^{+}-\mathrm{N}_{\mathrm{T}}^{-}\right)$versus the probe depth $(\varepsilon)$ into the energy gap as depicted in Fig. (3-20). The doping and the bad tailing width for poly-Si:H samples have the same trend as the charged defect density, Fig.(3-6). The change of the trap state density difference and band 
width with the deposition rate may be due to a change in the number of grain boundaries or due to a change of the electrical properties of the grain boundaries. Also, the relaxation time $\tau_{2}$ has a shortest value at the same deposition rate $(\approx 90 \AA / s)$ and increases with other deposition rates. This means that at this deposition condition we can prepare a high quality materials with a low trap state density close to the conduction band and a short relaxation time which lead to good electronic properties as also seen in the photomixing measurements. Trap state difference and charged density of states give similar results. This also gives the important message that both descriptions support each other.

\subsubsection{The hot wire chemical vapor deposition a-SiGe:H samples}

The transient voltage of the a-SiGe:H samples has two distinguishable decaying peaks as we see in Fig.(3-22). These two peaks appear separately but inside of the same transient regime. If the corresponding traps would fill completely and simultaneously, the transients would overlap. Therefore we suspect a refilling process of the second trap state. This means that the excess carriers after the stage 1 start to fill the (1) trap states first, they should be closest to the conduction band. However, there are some excess carriers, which fill the (2) trap states, which supposedly lie deeper. The first decaying peak has a larger amplitude $(\approx X 3)$ than the second one. Probably because of the majority of the carriers fill the first trap state which is closer to the conduction band edge than the second one. Also, when we calculated the trap state density difference from the two decaying peaks of the transient voltage, we found that there are indeed two peaks at two different energy positions inside the gap. With the first trap state density difference peak indeed closer to the conduction band than the second. It also has a larger amplitude than the second one. The different energy position and amplitude of the two trap state difference peaks support our argument of a refilling process of the second trap state. From the experimental and calculated relaxation time of the two decaying peaks, we also found that the relaxation time of the first peak is faster than the second one. This means that fast carriers fill the first trap states first and then carriers, which have a different mobility and velocity fill the second trap state. Some of these could indeed arrive after having been trapped in the first state. Finally we conclude that we obtain almost the same 
information about the trap state density difference from either one of the two decaying peaks. Two consecutive transients do not appear with poly-Si:H and thus are probably due to the compositional disorder or even due to specific clusters inside the a-SiGe:H alloys. Raman experiments on SiGe:H alloys have indeed shown specific clustering via a continuation and overlap of the Ge- and $\mathrm{Si}$ - oscillatory modes dispersion towards the $\mathrm{Si}$ and Ge- rich side of the alloy system, respectively[123].

\subsubsection{The low pressure reactive ECR plasma $\mu \mathrm{c}-\mathrm{SiGe:H}$ samples.}

The transient voltage signal of the $\mu \mathrm{c}-\mathrm{SiGe}: \mathrm{H}$ samples is in particular interesting because it has negative values like n-doped c-Si samples. Such a negative voltage, which should also be a measure of the trap state density difference against $\varepsilon=2 \mathrm{kT}$, cannot be measured for poly-Si:H nor for a-SiGe:H samples; this is to be expected for the case of symmetric band tailing and no specific doping. The $\mu \mathrm{c}-\mathrm{SiGe}: \mathrm{H}$ samples, however, have two negative peaks which can be assigned to two trap states just like with the a-SiGe:H samples. These two peaks also have about the same energy position inside the gap and may be part of it have doping character. However, the peaks of the $\mu \mathrm{c}-\mathrm{SiGe}: \mathrm{H}$ samples have about $80 \%$ amplitude less than those of the a-SiGe:H samples. This big difference in the amplitudes of the trap state density difference suggests a small amount of noncrystallinity in the $\mu \mathrm{c}-\mathrm{SiGe}: \mathrm{H}$ samples, i.e. a substantial decrease of the disorder inside the samples as compared to the a-SiGe:H samples, as expected. This also means that the total trap state density inside the $\mu \mathrm{c}-\mathrm{SiGe}: \mathrm{H}$ samples is less than that of a-SiGe:H samples; as also expected. The width of the trap density difference peaks; Fig.(3-35), has the same trend as the charged defect density that obtained from the photomixing measurements, Fig.(3-14c). This means that the two measurements support each other.

\subsection{The photoluminescence and SAXS characterizations}

The relative photoluminescence intensity at $80 \mathrm{~K}$ is correlated to the optical gap as seen in Table (3-1) and as expected. A higher Ge content (L743) reduces the optical gap while the defect density increases only slightly relative to L744. According to photomixing, the relative charge defect density increases only slightly with increasing the optical gap (Tauc gap), too. This increase of the defect density may be due to both 
structural changes and the creation of defects in the nonhomogeneous film. When we modeled the SAXS data using spherical objects, an average diameter $<\mathrm{D}>$ was obtained as the cause of the heterogeneity in the films. The values for $\angle \mathrm{D}>$ for these films are in Table (3-2) and are only somewhat smaller than the length of the LRPF deduced from the photomixing technique. From SAXA, the $\mathrm{Q}_{\mathrm{N}}$ that correlates to changes in the nonuniformity of the film due to nanovoids or Ge concentration fluctuations. $Q_{N}$ increases with increasing the Tauc gap as the charge defect density as measured from the photomixing technique. This means that the non-uniformity and defects in the film increase with increasing the optical gap (Tauc gap), which correlates the photoluminescence and photomixing results.

\subsection{The correlation between the photomixing and transient thermoelectric effects measurements}

In this work, we study the charge transport properties of poly-Si:H, a-SiGe:H and $\mu \mathrm{c}-$ $\mathrm{SiGe}: \mathrm{H}$ materials that are very promising materials in the field of photovoltaics. For that we use both the photoconductivity frequency mixing and the transient thermoelectric effects techniques. It is clear that there must be some correlation between the physical parameters, which are obtained from both methods.

In poly-Si:H materials, the photoconductivity, drift mobility and lifetime that measured from the photomixing have a peak at $\approx 90 \AA / \mathrm{s}$ due to the lower values of both the charge defect density and the doping and band tailing width at this deposition rate. This means that the charge defect density is related to the trap state density difference; in fact they are almost proportional.

In a-SiGe:H, the trap state density difference which is calculated from the two transient decaying peaks of the TTE increases with increasing Tauc gap just like the charge defect density as obtained from the photomixing technique. This is reflected in the decay of the photoconductivity, drift mobility and lifetime with increasing Tauc gap as seen in chapter 2 .

In the $\mu \mathrm{c}-\mathrm{SiGe}: \mathrm{H}$ materials the charge defect density has peak at the deposition rate $0.8 \AA / \mathrm{s}$ also the width of the trap state density difference peak has a maximum at the same deposition rate. 
The photomixing lifetime $\tau_{\mathrm{p}}$ is related to the photo-response, which corresponds to the TTE-stage 1, which we have not measured. The relaxation time $\tau_{2}$ is the ambipolar charge (stage 2) relaxation time. So the two relaxation times are different in the meaning and correspond to two different phenomena. This is evident already from the largely different time scales: the photomixing lifetime is in the order of nanoseconds and the relaxation time $\tau_{2}$ is in order of milliseconds. Thus we cannot expect a correlation between $\tau_{\mathrm{p}}$ and $\tau_{2}$ and indeed none is observed.

From the photoluminescence and SAXS measurements for the a-SiGe:H alloys, we find that the defects and the non-uniformity of the film increase with increasing optical gap (Tauc gap). That correlates the relative charge defect density which obtained from the photomixing to the optical gap. Probably there are Ge and Si clusters beyond a statistical mixture of Si and Ge. 


\section{Conclusion}

The charge transport properties of hydrogenated polycrystalline silicon (poly$\mathrm{Si}: \mathrm{H})$ films prepared by the hot-wire chemical vapor deposition (HWCVD) process as a function of deposition rate have been investigated in detail. A large amount of the work was done by employing the photoconductive frequency mixing technique. In particular, strong evidence for the existence of long-range potential fluctuations in poly-Si:H films has been found from the measurements of electric field dependence of the drift mobility. It was found that the charge transport properties peak at a deposition rate of $\approx 90 \AA / \mathrm{s}$. On the other hand, the depth and length of the long-range potential fluctuation plus the charged defect density have a minimum value at this deposition rate. The deterioration of the opto-electronic properties at other deposition rates may be due to increasing grain boundary effects. That makes the photomixing method a valuable tool for material quality testing.

The transient thermoelectric effect measurements were employed with the poly$\mathrm{Si}: \mathrm{H}$ films. From the relaxation amplitude and time of the stage 2, the trap state density difference can be calculated. It was found that the trap states density difference also has a minimum value at the deposition rate $\approx 90 \AA / \mathrm{s}$. That makes the TTE also a material discriminative tool.

The charge transport properties of hydrogenated amorphous silicon-germanium alloys (a-SiGe:H) alloys as prepared by the hot-wire chemical vapor deposition (HWCVD) process was investigated by employing the photoconductive frequency mixing technique. Again, evidence for the presence of long-range potential fluctuations in a-SiGe:H alloys is revealed from the measurements of electric field dependence of the drift mobility. The effect of the long-range potential fluctuations is enhanced when one increases the Tauc gap. That usually results in the deterioration of the opto-electronic properties. Specifically, through the drift mobility field dependence, the depth and length of the potential fluctuations as a function of Tauc gap are determined, and subsequently the charged defect density.

It was also found that the photoresponse decreases monotonically with increasing Tauc gap due to the decreases in the drift mobility and lifetime as a result of an increase in the concentration of charged defects around microvoids. 
The trap state density difference of the a-SiGe:H films is obtained from the decay profile of the transient thermoelectric effects measurements. This quantity also increases with increasing Tauc gap.

The low pressure reactive ECR plasma $\mu \mathrm{c}-\mathrm{SiGe}: \mathrm{H}$ materials have a high quality and favorable transport properties as compared to samples made with other techniques. Their electronic transport properties are measured by both the photomixing and transient thermoelectric effects for the first time. The photoresponse has maximum value at the deposition rate $\approx 0.8 \AA / \mathrm{s}$. Probably because of a minimum value of the trap state density difference at this deposition rate.

In summary:

1. The photomixing technique is capable of measuring both the drift mobility, lifetime and charged defect density of the poly-Si:H, a-SiGe:H and $\mu c-\mathrm{SiGe}: \mathrm{H}$ samples.

2. We confirm that the long-range potential fluctuations model works for the charge transport properties; also for materials optimization.

3. The transient thermoelectric effects technique can be used to measure the relaxation time and magnitude of the space charges in these materials, and is also useful for quality inspection.

4. The different trap state density can also be calculated from the transient thermoelectric effects measurements. Evidence for cluster electronic states in aSiGe:H and $\mu \mathrm{c}-\mathrm{SiGe}: \mathrm{H}$ films is found.

5. According to the photoluminescence and SAXS measurements, the nonuniformity and defects in the a-SiGe:H alloys increase with increasing optical gap (Tauc gap). That is confirmed by both photomixing (charge defect density) and transient thermoelectric effects (trap state density difference) measurements.

6. These materials are promising in the solar energy field and we hope that in the future we can make more analysis for the composite semiconductor materials like $\mathrm{Si} / \mathrm{Ge}$. 


\section{References}

1. W.E. Spear and P.G. Le Comber, Philos.Mag.33 (1976) 935.

2. D.E. Carlson and C.R. Wronski, Appl. Phys. Lett.28 (1976) 671.

3. P.G. Le Comber and W.E. Spear, in semiconductor and semimetals, edited by J. PanKore (Academic, New York, 1984), Vol.21, Part D, chater6.

4. D. kruangam, T. Endo and W. guang-Pu, Jpn. J. Appl.Phys.Part2 24(1985) L806.

5. H.K. Tsai and S.C. Lee, Appl.Phys.Lett.52 (1988) 275.

6. W. J .Sah, S. C. Lee, and J. H. Chou, Proceeding of 1989 International Electron Device Meeting, Washington D.C., USA, (1989) 515.

7. A. Matsuda, T. Yamaoka, S. Wolff, M. Koyama, Y. Imanishi, H. Mataurra, and K. Tanaka, J. Appl. Phys. 60 (1986) 4025.

8. D. A. Anderson and W. E. Spear, Philos. Mag. B 35 (1977) 1.

9. W. L. Lin, H. K. Tsai, S. C. Lee, W. J. Sah, and W. J. Tzeng, Appl. Phys. Lett.51 (1987) 2112.

10. H. K. Tsai, W. L. Lin, and S. C. Lee, J. Appl. Phys. 64 (1988) 1910.

11. J. H. Chen, W. T. Sah, and S. C. Lee, J. Appl. Phys. 70 (1991) 125.

12. K. D. Mackenzie, J. R. Eggert, D. J. Leopold, Y. M. Li, S. Lin, and W. Paul, Phys. Rev. B 31 (1985) 2198.

13. G. Lucovsky, S. S. Chao, J. Yang, J. E. Tyler, R. C. Ross, and W. Czubatyj, Phys. Rev. B 31 (1985) 2190.

14. M. Cardona, Phys. Status Solidi B 118 (1983) 463.

15. J. Y. W. Seto, J. Appl. Phys. 46 (1975) 5247.

16. G. Baccarani, B. Ricco, and B. Spadini, J. Appl. Phys. 49 (1978) 5565.

17. T. Kamins, Polycrystalline Silicon for Integrated Circuits and Displays, 2nd ed. (Kluwer, Boston, 1998).

18. G. J. Korsh and R. S. Muller, Solid-State Electron. 21 (1978) 1045. 
19. N. C.C. Lu, L. Gerzberg, C.-Y. Lu, and J. D. Meindl, IEEE Trans. Electron Devices ED-30 (1983)137.

20. P. G. LeComber, J. Non-Cryst. Solids 115 (1989) 1.

21. T. Matsuyama, M. Tanaka, S. Tsuda, S. Nakano and Y. Kuwano, Jpn. J. Appl. Phys. 32 (1993) 3720.

22. H. Matsumura, J. Appl. Phys. 65 (1984) 4396.

23. N. Matsuo, T. Sugiyama and T. Miyoshi, Jpn. J. Appl. Phys. 35(1996) L1215.

24. M. Nakata, A. Sakai, T. Uematsu, T. Namikawa, H. Shirai, J. Hanna and I. Shimizu, Phil. Mag. B 63(1991) 87.

25. T. J. Donahue and R. Reif, J. Appl. Phys. 57(1985) 2757.

26. M. Cuniot, J. Dixmier, P. Elkuim, E. Bardel and J. E. Bour'ee, 12th European Photovoltaic Solar Energy Conf.(Amsterdam) (1994) p 1819.

27. Tringe. J.W. and Plummer J. D., J. Appl. Phys., 87(11) (2000) 7913

28. J.C. Sturm, E.J. Prinz and C.W. Magee, IEEE Electronic Device Lett. 12 (1991) 303.

29. T.L. Lin, T. George, E.W. Jones, A. Ksenzov and M.L. Humberman, Appl. Phys. Lett. 60(1992) 380.

30. A. Terakawa, M. Shima, K. sayama, H. Tarui, H. Nishiwaki and S. Tsuda, Jpn. J. Appl. Phys. 34(1995) 1741.

31. A. Terakawa, M. Shima, K. sayama, H. Tarui, S. Tsuda, and S. Nakano, Jpn. J. Appl. Phys. 32 (1993) 4849.

32. W.Paul, D.K. Paul, J. Blake, B.VonRedern, J.Blake and S. Oguz, Phys. Rev. Lett. 46 (1981) 1016.

33. B. VonRedern, D.K. Paul, J. Blake, R.W. Collins, G. Moddel and W. Paul, Phys. Rev. B 25 (1982) 7678.

34. S. Z. Weisz, N. Gomes, J.A. Muir, O. Resto and R. Perez, Appl. Phys. Lett. 44(1984) 634. 
35. Chevalier J, Wieder H, Onton A and Crournier C R, Solid State Commun. 24 (1977) 867.

36. A. R. Middya, S. Hazra and S. Ray, J. Appl. Phys. 76 (1994) 7578.

37. A. Matsuda and K. Tanaka, J. Non-Cryst. Solids 97 \& 98(1987) 1367.

38. S. Guha, J. S. Payson, S. C. Agarwal and S. R. Ovshinsky, J. Non-Cryst. Solids 97 \& 98 (1987) 1455.

39. K. D. Mackenzie, J. R. Eggert, D. J. Leopold, Y. M. Li, S. Lin and W. Paul, Phys. Rev. B 31(1985) 2198.

40. S. Hazra, A. R. Middya and S. Ray, J. Non-Cryst. Solids 211(1997) 22.

41. A. Matsuda, K. Nomoto, Y. Takuchi, A. Suzuki, A. Yuuki and J. Perrin, Surf. Sci. 227(1990) 50.

42. H. Matsumura, Jpn. J. Appl. Phys. 25/2 (1986) L949.

43. C. Horbah, W. Beyer, H. Wagner, J. Non-Cryst. Solids 137-138 (1991) 661.

44. H. Matsumura, Appl. Phys. Lett. 51(11) (1987) 804.

45. M. Lill, B. Schroder, Appl. Phys. Lett. 74 (1999) 1284.

46. C. Berge, R.B. Bergmann, T.J. Rinke, J.H. Werner, Proceedings of the $17^{\text {th }}$ European Photovoltaic Solar Energy Conference, Munich (2001) 1277.

47. A.V. Shah, J. Meier, L. Feitknecht, E. Vallat-Sauvain, J. Bailat, U. Graf, S. Dubail, C. Droz, Proceedings of the $17^{\text {th }}$ European Photovoltaic Solar Energy Conference, Munich (2001) 2823.

48. J. Kanicki: Amorphous and Microcrystalline Semiconductors Device: Optoelectronic Devices (Artech House, Boston, London, 1991) Chapter 1.

49. J. Kanicki: Amorphous and Microcrystalline Semiconductors Device: Materials and Device Physics (Artech House, Boston, London, 1991).

50. M. Shima, A. Terakawa, M. Isomura, M. Tanaka, S. Kiyama and S. Tsuda, Appli. Phys. Lett. 71(1997) 84. 
51. N. Saxena, D. E. Albright, C.M. Fortmann, T.W. Fraser Russell, P.M. Fauchet and I.H. Campbell, J. Non-Cryst. Solids 114(1989) 801.

52. J. Xu, S. Miyazaki, and M. Hirose, J. Non-Cryst. Solids 208(1996) 277.

53. G. Ganguly, T. Ikeda, T. Nishimiya, K. Saitoh, M. Kondo and A. Matsuda, Appl. Phys. Lett. 69(1996) 4224.

54. G. Ganguly, M. Fukawa, T. Ikeda and A. Matsuda, J. Non-Cryst. Solids 227230(1996) 1069.

55. H.F. Sterling, and R.C.G. Swann, Solid-state electron. 8 (1965) 653.

56. J. Perrin, in: plasma deposition of amorphous-based materials, eds. G. Bruno, P. Capezzuto, and A. Madan (Academic press Inc., San Diego, CA, 1995) 216.

57. W. Luft and Y. S. Tsuo, Hydrogenated amorphous silicon alloy deposition processes, Applied Physics Series 1 ( marcel Dekker, Inc., New York, Basel, Hong Kong, 1993).

58. G. Burno, P. Capezzuto, and A. Madan, plasma deposition of amorphous-based materials ( Academic press, Inc. 1995).

59. K.F. Feenstra, C.H.M. van derWerf, E.C. Molenbroek and R.E.I. Schropp, Deposition of device quality amorphous silicon by hot-wire CVD, in: Amorphous and Microcrystalline Silicon Technology- 1997, edited by S. Wagner, M. Hack, E.A. Schiff, R. Schropp, and I. Shimizu, Materials Research Society Symp. Proc. 467 (1997) 645 .

60. E.C. Molenbroek, A. H. Mahan, E.J. Johnson, and A.C. Gallagher, J. Appl. Phys. 79 (1996) 7278 .

61. F. Jansen, I. Chen and M.A. Machonkin, J. Appl. Phys. 66 (1989) 5749.

62. Y. Ichikawa, K. Aizawa, H. Shimabukuro, Y. Nagao and H. sakai, Technical Digest of the international PVSEC-3, Tokyo, Japan (1987) A-Ip-5.

63. Y.H. Shing, Solar cells 27 (1989) 331.

64. Y. Hattori, D. Kruangam, k. Katoh, Y. Nitta, H. Okamoto and Y. Hamakawa, Proc. of the $19^{\text {th }}$ IEEE PV Specialists Conf., (1987) 689. 
65. V.L. Dalal, E.X. ping, S. Kaushal, M. Bhan and M. Leonard, Appl. Phys. Lett. 64 (1994) 1862.

66. V.L. Dalal, T. Maxson, R. Girvan, and S. Haroon, In Amorphous and microcrystalline silicon technology-1997, edited by S. Wagner, M. Hack, E.A. Schiff, R. Schropp and I. Shimizu, Mater. Res. Soc. Symp. Proc. 467 (1997) 813.

67. K. Kurihara, K. Sasaki, M. Kawarada, and N. Koshino, Appl. Phys. Lett. 52 (1988) 437.

68. R.J. Severens, M.C.M. van der Sanden, H.J.M. Verhoeven, J. Bastiaanssen and D.C. Schram, in Amorphous silicon technology- 1996, edited by M. Hack, E.A. Schiff, S. Wagner, R. Schropp and A. Matsuda, Mater. Res. Soc. Symp. Proc. 420 (1997) 341.

69. E.R. Giessinger, R. Braunstein, S. Dong and B.G. Martin, J. Appl. Phys. 69, (1991) 1469.

70. Yi Tang, R. Braunstein and B. Von Roedern, Mater. Res. Soc. Symp. Proc. 258, (1992) 735 .

71. Yi Tang, R. Braunstein, B. Von Roedern, and F.R. Shapiro, Mater. Res. Soc. Symp. Proc. 297, (1993) 407.

72. R. Braunstein and Yi Tang, Proceeding of the $21^{\text {st }}$ International conference on Physics of Semiconductors (August 1992), Beijing, China, 1,269.

73. Yi Tang, R. Braunstein and B. Von Roedern, Appl. Phys. Lett. 63(17)(1993) 2393.

74. R. Brüggemann, C. Main, J. Berkin and S.Reynolds, Phil.Mag.B62 (1990) 29.

75. H. Oheda, J.Appl. Phys. 52 (1981) 6693.

76. Yi Tang, thesis, UCLA 1992.

77. A. Kattwinkel, thesis, Göttingen 2001.

78. J. Liebe, K. Heinemann, K. Bärner, I.V. Medvedeva, Yi Tang, S. Dong, R. Braunstein, Materials Science and Engineering B47 (1997) 244.

79. M. S. Brandt, M. W. Bayer, J. Non-Cryst. Solids 266-269 (2000) 1.

80. K. Seeger, Semiconductor physics, Springer, New York, (1973) 156. 
81. R.N. Hall, Phys. Rev. 87(1952) 387.

82. W. Shockley, W. T. Read, Phys. Rev. 87 (1952) 835.

83. M. Sasaki, H. Negeshi, M. Inoue, J. Appl. Phys. 59 (1986) 796.

84. M. Sasaki, H. Negeshi, M. Inoue, J. Appl. Phys. 61(1987) 2267.

85. W. van Roosbroeck, Phys. Rev.15 (1953) 282.

86. C. Kittel, Introduction to solid state physics, $3^{\text {rd }}$ edn., Wiley, New York,(1967) P.307

87. E. Spenke, Elehtron Halbleiter, $2^{\text {nd }}$ edn., Springer, Berlin, (1965) P.424.

88. J. Liebe, A. Kattwinkel, K. Bärner, G. Sun, S. Dong, R. Braunstein, Mater. Sci. Eng. A282 (2000) 158.

89. A. Kattwinkel, E. Kraus, Ch. Kleeberg, T. Gron, K. Bärner, E. Schmitt, E. Gmelin, Aca. Physica Polonica A 93 (1998) 745.

90. E. Kraus, diploma thesis, Göttingen 1995.

91. N. F. Mott and E.A. Davies, "Electronic Processes in Non-Crystalline Materials", Clarendon, Oxford 1979.

92. L.A. Fegin, D.I. Svergun, Structure Analysis by Small-Angle X-Ray and Neutron Scattering (Plenum, New York, 1987).

93. J.E. Epperson, P. Thyagarajan, J. Appl. Crystalogr.,21(1989) 652.

94. G. Georigk, D.L. Williamson, H. G. Haubold, HASYLAB Annual Report (1996) 628.

95. D.L. Williamson, Mater. Res. Soc. Symp. Proc., 377(1995) 251.

96. R.J. Temkin, W. Paul, and G. A. N. Connell, Ad. Phys., 22(1973) 581.

97. H. Stabinger, and O. Kratky, Makromol. Chem., 179(1978) 1655.

98. Y. Xu, B.P. Nelson, L.M. Gedvilas, R.C. Reedy, Thin Solid Films 430 (2003) 197.

99. A. Terakawa et al, Jpn. J. Appl. Phys. 34 (1995) 1741.

100. V.L. Dalal, S. Haroon, Z. Zhou, T.Maxson, K.Han, J. Non-Cryst. Solids, 266269(2000) 675. 
101.V. L. Dalal, E. X. Ping, S. Kaushal, M.K. Bhan, and M.Leonard, Appl. Phys. Lett. 64 (1994) 1862.

102. S.R. Sheng, X.B. Liao and G.L. Kong, Appl. Phys. Lett. 78 (2001) 2509.

103.S. Dong, J. Liebe, Y. Tang, R. Braunstein and B. von Roedern, NREL/SNL Photovoltaics Program Review Meeting, AIP Conf. Proc. 344 (1996) p.537.

104. A. Howard and R.A. Smith, Phys. Rev. B 44 (1991) 7935

105. Y. Tang and R. Braunstein, J. Appl. Phys.79 (1996) 850.

106. Y. Tang, S. Dong, R. Braunstein, B. von Roedern, Appl. Phys. Lett. 68(1996) 640.

107.B. von Roedern and A. Madan, Phil. Mag., B 63 (1991) 293.

108.H. Overhof, and P. Thomas, Electronic Transport in Hydrogenated Amorphous Semiconductors, Springer Tracts in Modern physics, vol.114 (Berlin, Springer, 1989).

109.D.L. Williamson, Y. Xu, and B.P. Nelson, M. Al-Jassim, J.P. Thornton, and J.M. Gee, NCPV Photovoltaics Program Review, AIP Conf. Proc. No. 462, AIP, Woodbury, NY (1999) 272.

110. G. Goerigk and D.L. Williamson, J. Appl. Phys. 90 (2001) 5808.

111.H. Matsumura, Mat. Res. Soc. Symp. Proc. vol. 118 (1988) 541

112.J.W. Trnge and J.D. Plummer, J. Appl. Phys., 87(11), (2000) 7913.

113. S.R. Sheng, G.S. Sun, J. Liebe, A. Kattwinkel, R. Braunstein, B.P. Nelson, B. von Roedern , K. Bärner, Mater. Sci. Eng. A325 (2002) 490.

114.K.D. Mackenzie, J.R. Eggert, D.J. Leopold, Y. M. Li, S. Lin, and W. Paul, Phys. Rev. B 31, 2198 (1985).

115. S.J. Jones, Y. Chen, D.L. Williamson, R. Zedlitz and G. Bauer, Appl. Phys. Lett. 62 3267 (1993).

116. W. Beyer, H. Wagner, and F. Finger, J. Non-Cryst. Soilds, 77\&78, (1985) 857.

117.W. Luft, Proc. $20^{\text {th }}$ IEEE Photovoltaic Specialist's Conf. IEEE, 1988, p.218.

118. C.M. Fortmann, Mat. Res. Soc. Symp. Proc. 192, 27 (1990). 
References

119. G.Z. Yue, X.M. Deng, G. Ganguly, and D. Han, J. Non-Cryst. Solids, 266, part B (2000).

120. S.R. Sheng, M. Boshta, R. Braunstein, V.L. Dalal, J. Non-Cryst. Solids 303 (2002) 201.

121. G.H. Bauer, F. Voigt, R. Carius, M. Krause, R. Brueggemann, T. Unold, J. NonCryst. Solids 299-302 (2002) 153.

122. S. Huang, K. Chen, J. Shi, X. Huang, J. Xu, G. Ganguly, A. Matsuda, Jpn. J. Appl. Phys., 40(2001) 40.

123. S.R. Jadkar, J.V. Sali, S.T. Kshirsagar, M.G. Takwale, J. Non-Cryst. Solids 299-302 (2002) 168 


\section{Acknowledgements}

I am deeply grateful to Prof. Dr. K. Bärner for his constant and inexhaustible help, and I truly thank him for the big scientific freedom he always provided, without which most of this work would not exist.

I'm also in debt to Prof. R. Braunstein; at Physics and Astronomy department, University of California at Los Angeles (UCLA); for his offering me to learn the photomixing technique and his support to this topic.

I would like to send my thanks to Barakat, Alavi and Victor Guerrero for their helping me during the time in UCLA. Also they created a family life in UCLA.

I address my thanks to the members of the group, Dr. A. Kattwinkel, N. Hamad, I. Elkassab, V. Morchshakov and W. Hahn for their valuable help in the experimental work and for the nice atmosphere they created inside the group. I am grateful to the Herrn Frenzel, head of the mechanics workshop, Herrn Langohr, head of the electronic workshop, and all their coworkers for the inistant, competent and prompt job.

Frau E. Rohrmoser, M. Ackermann and Frau H. Afshar, are kindly acknowledged for their continuous help with all the small and big administrative problems that a foreigner always has in a foreign country.

My sincere thanks to all the members of the IV.Physikalisches Institut in Göttingen, who always make the working atmosphere enjoyable and relaxed.

Finally, my thoughts inevitably go to my parents, my family and to all those persons who always, critically, constructively supported my choices and my work. To them I address my deepest gratitude and the best wishes for a brilliant continuation. I would like to thank and highly appreciate the Egyptian Government for financing my study program.

Göttingen, 2003 


\section{Lebenslauf}

\begin{tabular}{|c|c|}
\hline Familienname & Boshta \\
\hline Vorname & Mostafa Abd ElMoemen Hassan \\
\hline Nationalität & Ägypter \\
\hline Geburtsdatum & 01. Oct., 1970 \\
\hline Geburtsort & Ägypten - EL-Menofia \\
\hline Familienstan & ledig \\
\hline Addresse & IV.Physiklishes Institut, Tammann Str.1, 37077 Göttingen \\
\hline $1976-1981$ & Primary school \\
\hline $1981-1984$ & Preparatory school \\
\hline $1984-1987$ & Secondary school \\
\hline $1987-1991$ & Aufnahme des Physikstudiums an der Menoufia Universität \\
\hline Jan., 1992 - März,1993 & Wehrdienst \\
\hline März 1997 & Diplomprüfung Physik an der Ain Shams Universität \\
\hline Die Thema & $\begin{array}{l}\text { Physical and spectral study for tin oxide }\left(\mathrm{SnO}_{2}\right) \text { thin film } \\
\text { prepared by spray pyrolysis. }\end{array}$ \\
\hline seit January 2000 & $\begin{array}{l}\text { Promotion am IV.Physikalisches Institut (Institut für } \\
\text { Halbleiterphysik) der Georg-August- universität Göttingen } \\
\text { unter der wissenschaftlichen Leitung von Herrn Prof. Dr. K. } \\
\text { Bärner, Finanzierung der Doktorarbeit durch stipendium des } \\
\text { Landes Ägypten }\end{array}$ \\
\hline März -Dezember 2001 & Forschungstätigkeit im Rahmen der Promotion an der \\
\hline April-September 2002 & $\begin{array}{l}\text { University of California, Los Angeles, Department of Physics } \\
\text { and Astronomy in der Arbeitsgrouppe von Prof. Dr. R. } \\
\text { Braunstein. }\end{array}$ \\
\hline
\end{tabular}

